\title{
Brain-Machine-Brain Interface
}

by

\author{
Joseph E. O’Doherty \\ Department of Biomedical Engineering \\ Duke University
}

Date:

Approved:

\begin{tabular}{c}
\hline Miguel A. L. Nicolelis, Supervisor \\
\hline Craig Henriquez \\
\hline Patrick Wolf \\
\hline Dennis Turner
\end{tabular}

Dissertation submitted in partial fulfillment of the requirements for the degree of

Doctor of Philosophy in the Department of Biomedical Engineering in the Graduate School of Duke University

2011 


\author{
ABSTRACT \\ (Biomedical engineering) \\ Brain-Machine-Brain Interface \\ by \\ Joseph E. O’Doherty \\ Department of Biomedical Engineering \\ Duke University
}

Date:

Approved:

Miguel A. L. Nicolelis, Supervisor

\begin{tabular}{c}
\hline Craig Henriquez \\
\hline Patrick Wolf \\
\hline Dennis Turner
\end{tabular}

An abstract of a dissertation submitted in partial fulfillment of the requirements for the degree of Doctor of Philosophy in the Department of Biomedical Engineering in the Graduate School of Duke University

2011 
Copyright (C) 2011 by Joseph E. O’Doherty

All rights reserved except those rights granted by the Creative Commons Attribution-NonCommercial-NoDerivs 3.0

United States License (CC BY-NC-ND 3.0). 


\section{Abstract}

Brain-machine interfaces (BMIs) use neuronal activity to control external actuators. As such, they show great promise for restoring motor and communication abilities in persons with paralysis or debilitating neurological disorders.

While BMIs aim to enact normal sensorimotor functions, so far they have lacked afferent feedback in the form of somatic sensation. This deficiency limits the utility of current BMI designs and may hinder the translation of future clinical BMIs, which will need a means of delivering sensory signals from prosthetic devices back to the user.

This dissertation describes the development of brain-machine-brain interfaces (BMBIs) capable of bidirectional communication with the brain. The interfaces consisted of efferent and afferent modules. The efferent modules decoded motor intentions from the activity of populations of cortical neurons recorded with chronic multielectrode recording arrays. The activity of these ensembles was used to drive the movements of a computer cursor and a realistic upper-limb avatar. The afferent modules encoded tactile feedback about the interactions of the avatar with virtual objects through patterns of intracortical microstimulation (ICMS).

I first show that a direct intracortical signal can be used to instruct rhesus monkeys about the direction of a reach to make with a BMI. Rhesus monkeys placed an actuator over an instruction target and obtained, from the target's artificial texture, information about the correct reach path. Initially these somatosensory instructions took the form of vibrotactile stimulation of the hands. Next, ICMS of primary somatosensory cortex $\left(\mathrm{S}_{1}\right)$ 
in one monkey and posterior parietal cortex (PPC) in another was substituted for this peripheral somatosensory signal. Finally, the monkeys made direct brain-controlled reaches using the activity of ensembles of primary motor cortex (M1) cells, conditional on the ICMS cues. The monkey receiving ICMS of $\mathrm{S}_{1}$ was able to achieve the same level of proficiency with ICMS as with the stimulus delivered to the skin of the hand. The monkey receiving ICMS of PPC was unable to perform the task above chance. This experiment indicates that ICMS of S1 can form the basis of an afferent prosthetic input to the brain for guiding brain-controlled prostheses.

I next show that ICMS of $S_{1}$ can provide feedback about the interactions of a virtualreality upper-limb avatar and virtual objects, enabling active touch. Rhesus monkeys initially controlled the avatar with the movements of their arms and used it to search through sets of up to three objects. Feedback in the form of temporal patterns of ICMS occurred whenever the avatar touched a virtual object. Monkeys learned to use this feedback to find the objects with particular artificial textures, as encoded by the ICMS patterns, and select those associated with reward while avoiding selecting the non-rewarded objects. Next, the control of the avatar was switched to direct brain-control and the monkeys continued to move the avatar with motor commands derived from the extracellular neuronal activity of M1 cells. The afferent and efferent modules of this BMBI were temporally interleaved, and as such did not interfere with each other, yet allowed effectively concurrent operation. Cortical motor neurons were measured while the monkey passively observed the movements of the avatar and were found to be modulated, a result that suggests that concurrent visual and artificial somatosensory feedback lead to the incorporation of the avatar into the monkey's internal brain representation.

Finally, I probed the sensitivity of S1 to precise temporal patterns of ICMS. Monkeys were trained to discriminate between periodic and aperiodic ICMS pulse trains. The periodic pulse-trains consisted of $200 \mathrm{~Hz}$ bursts at a $10 \mathrm{~Hz}$ secondary frequency. The aperiodic pulse trains had a distorted periodicity and consisted of $200 \mathrm{~Hz}$ bursts at a variable instantaneous 
secondary frequency. The statistics of the aperiodic pulse trains were drawn from a gamma distribution with equal mean inter-burst intervals to the periodic pulse trains. The monkeys were able to distinguish periodic pulse trains from aperiodic pulse trains with coefficients of variation of 0.25 or greater. This places an upper-bounds on the communication bandwidth that can be achieved with a single channel of temporal ICMS in S1.

In summary, rhesus monkeys were augmented with a bidirectional neural interface that allowed them to make reaches to objects and discriminate them by their textures-all without making actual movements and without relying on somatic sensation from their real bodies. Both action and perception were mediated by the brain-machine-brain interface. I probed the sensitivity of the afferent leg of the interface to precise temporal patterns of ICMS. Moreover, I describe evidence that the BMBI controlled avatar was incorporated into the monkey's internal brain representation. These results suggest that future clinical neuroprostheses could implement realistic feedback about object-actuator interactions through patterns of ICMS, and that these artificial somatic sensations could lead to the incorporation of the prostheses into the user's body schema. 
To my parents 
We will be engaged in the development of principles and techniques by which information from the nervous system can be used to control external devices such as prosthetic devices, communications equipment, teleoperators ... and ultimately perhaps even computers.

[But] ...we are immediately going to have to provide transducers and stimulators so that ... information about a prosthetic device or a communication channel can be fed back into the nervous system ...

(Karl Frank, 1968) 


\section{Contents}

Abstract iv

List of Tables $\quad$ xiv

List of Figures $\quad$ Xv

List of Abbreviations and Symbols $\quad$ xviii

Acknowledgements $\quad$ xx

1 Introduction 1

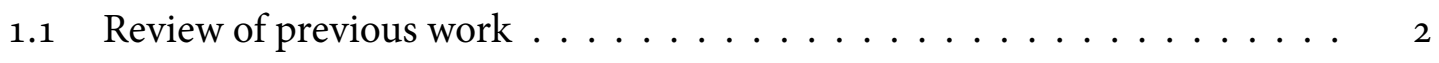

1.1.1 CNS motor neuroprosthesis $\ldots \ldots \ldots \ldots$

1.1.2 CNS sensory neuroprosthesis .................. 9

1.1 .3 Sensory neuroprosthesis ................. 10

1.1.4 Prosthetic somatosensation ................ 11

1.1.5 Bidirectional neuroprosthesis ............... 12

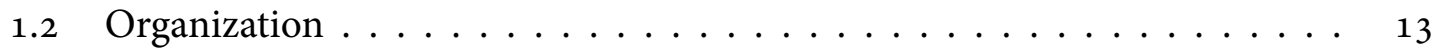

2 Experimental methodology $\quad 15$

2.1 Basic experimental procedures .................... 15

2.1.1 Animal models . . . . . . . . . . . . . . . . . . 15

2.1.2 Basic behavioral preparation . . . . . . . . . . . . 15

2.2 Cortical implants ..................... 16

2.2.1 Revision A electrode array . . . . . . . . . . . . . 16 
2.2.2 Revision B electrode array . . . . . . . . . . . . . 17

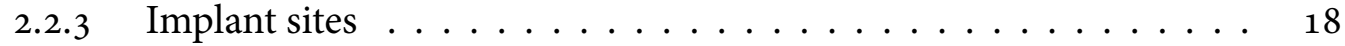

2.3 Electrophysiological recordings . . . . . . . . . . . . . . . . . . 19

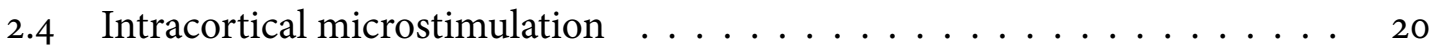

2.4.1 Stimulus isolation channels ............... 20

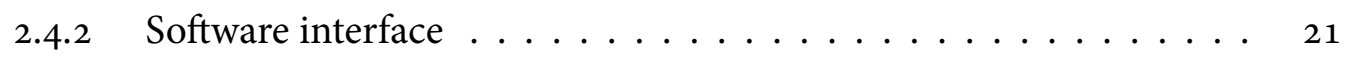

2.4 .3 Psychometric measurements ................ 23

2.5 Analysis methodology .................... 23

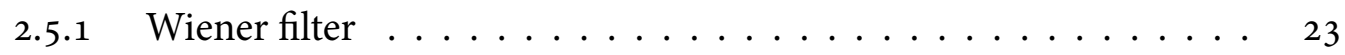

2.5.2 Unscented Kalman filter . . . . . . . . . . . . . . . . 24

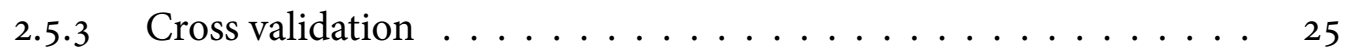

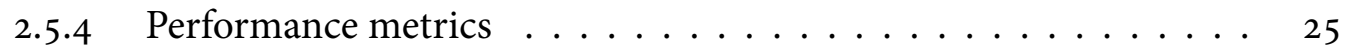

2.6 Real-time operations . . . . . . . . . . . . . . . 27

2.6.1 Data preprocessing ..................... 27

2.6 .2 Brain control ..................... 27

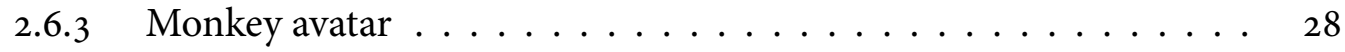

3 Sensory cue $\quad 30$

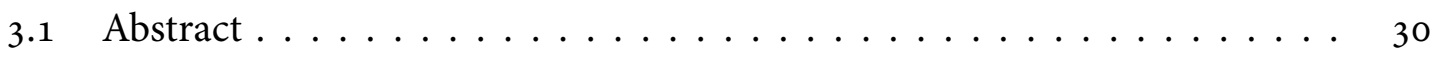

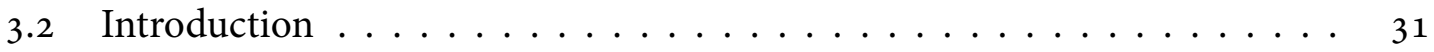

3.3 Material and methods $\ldots \ldots \ldots \ldots \ldots \ldots \ldots \ldots \ldots$

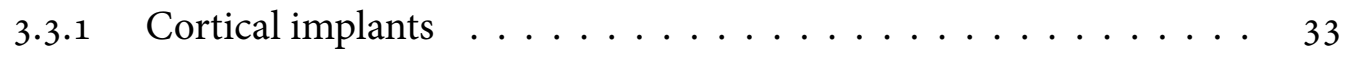

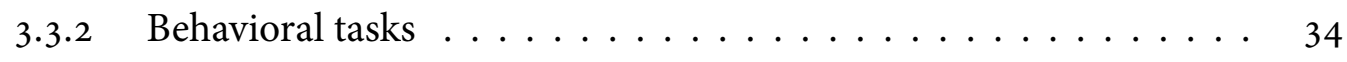

3.3 .3 Intracortical microstimulation $\ldots \ldots \ldots \ldots \ldots$

3.3 .4 Linear discriminant analysis . . . . . . . . . . . 38

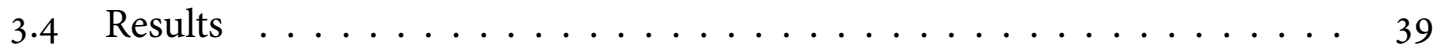


3.4.1 Ensemble activity during reaching tasks . . . . . . . . . 39

3.4 .2 Decoding movement $\ldots \ldots \ldots \ldots \ldots \ldots$

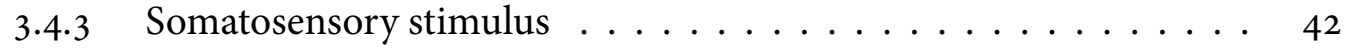

3.4 .4 BMI with somatosensory input $\ldots \ldots \ldots \ldots$

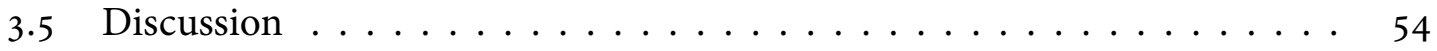

3.5 .1 Rate of learning ....................... 54

3.5.2 Mechanism of action of ICMS . . . . . . . . . . 55

3.5 .3 Ineffectiveness of PPC stimulation $\ldots \ldots \ldots \ldots$

3.5 .4 Sensory Substitution $\ldots \ldots \ldots \ldots \ldots \ldots$

4 Sensory feedback $\quad 58$

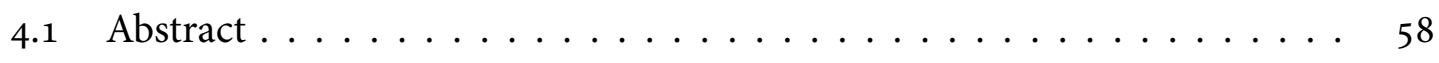

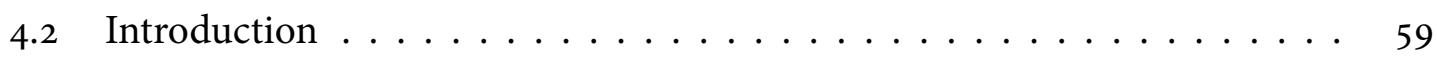

4.3 Materials and methods ................... 61

$4.3 .1 \quad$ Electrode arrays ................... 61

$4.3 .2 \quad$ Tasks ........................ 61

$4.3 .3 \quad$ Catch trials ......................... 63

4.3 .4 Algorithms ...................... 63

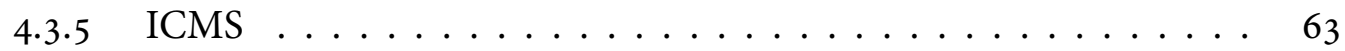

4.3 .6 Interleaved ICMS and recordings $\ldots \ldots \ldots 63$

4.3 .7 Perievent time histograms (PETHs) . . . . . . . . . . . 64

4.3 .8 Directional tuning curves .................... 64

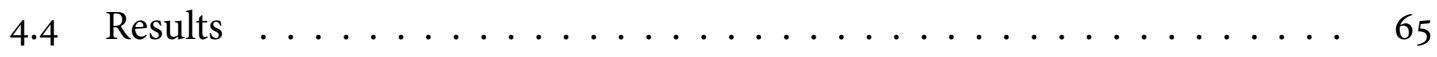

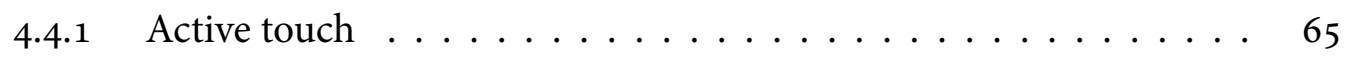

4.4.2 Brain-machine-brain interface ........... 73

4.4 .3 Neurophysiological findings . . . . . . . . . . . . . 75 
4.4 .4 Avatar incorporation .................... 79

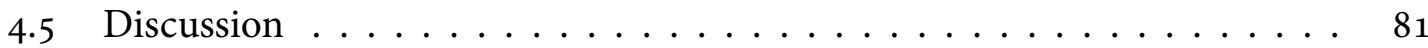

4.5 .1 Interleaved ICMS and recordings $\ldots \ldots \ldots . \ldots \ldots 2$

4.5 .2 Implementation in human subjects $\ldots \ldots \ldots . \ldots . \ldots 2$

4.5 .3 Prosthesis incorporation $\ldots \ldots \ldots \ldots \ldots$

5 Randomly patterned ICMS $\quad 84$

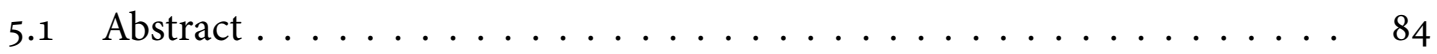

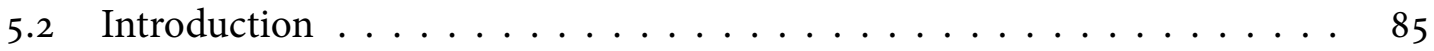

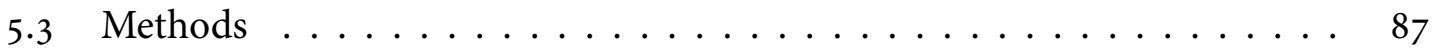

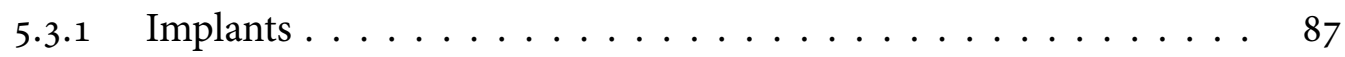

5.3 .2 Behavioral task ....................... 87

$5 \cdot 3.3 \quad$ ICMS patterns $\ldots \ldots \ldots \ldots \ldots \ldots \ldots$

5.3.4 Kinematics extraction and artifact suppression . . . . . . . . 91

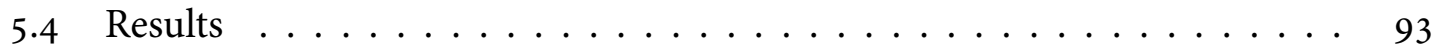

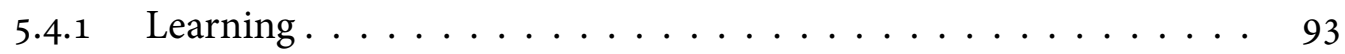

5.4 .2 Psychometrics ............................. 93

$5.4 .3 \quad$ Kinematics extraction . . . . . . . . . . . . . . 97

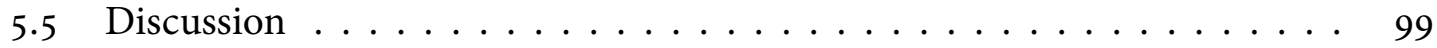

5.5 .1 Prosthetic somatosensation $\ldots \ldots \ldots \ldots \ldots$

5.5 .2 Artifact mitigation ....................... 101

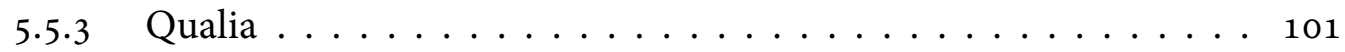

6 Discussion $\quad 103$

6.1 Engineering Contributions . . . . . . . . . . . . . . . . 104

6.1.1 Sensory input concurrent with BMI . . . . . . . . . . . 104

6.1.2 Interleaved stimulation and recordings . . . . . . . . . 104 
6.1.3 Controlled aperiodic patterns of stimulation . . . . . . . . . . 104

6.2 Unresolved questions . . . . . . . . . . . . . . . . 105

6.2.1 The question of qualia . . . . . . . . . . . . . 105

6.2.2 The timescales of learning of $S_{1}$ ICMS patterns $\ldots \ldots . . \ldots 105$

6.2 .3 Long-term effects of ICMS . . . . . . . . . . . . . . . . 106

6.2.4 Prosthesis incorporation into the body schema . . . . . . . . . 106

6.2.5 Requirement of somatotopy . . . . . . . . . . . . . . 107

6.2.6 Sensory substitution . . . . . . . . . . . . . . . . 107

6.2.7 Can any sensory cortical region be used as a target for ICMS? . . . 108

6.3 Future Work . . . . . . . . . . . . . . . . . . . . . 109

6.3.1 Improved artifact mitigation . . . . . . . . . . . . . . . . 109

6.3.2 Optogenetic ICMS for prosthetic somatosensation . . . . . . . . 110

6.3 .3 Representing texture with gratings ... . . . . . . . . 110

6.3 .4 Spatiotempral ICMS . . . . . . . . . . . . . . . . . . 112

6.3.5 Closed-loop control of ICMS parameters . . . . . . . . . . . 113

6.3.6 Proprioception and pain . . . . . . . . . . . 113

6.3.7 Incorporation of tactile sensors into physical devices . . . . . . 115

6.3.8 Avatar incorporation experiments in humans . . . . . . . . . 116

$\begin{array}{lr}\text { Bibliography } & 117\end{array}$

$\begin{array}{lr}\text { Biography } & 131\end{array}$ 


\section{List of Tables}

3.1 Average prediction accuracy for the sensory cue task $\ldots \ldots \ldots \ldots$ 


\section{List of Figures}

1.1 Schematic of the brain-machine-brain interface concept $\ldots \ldots \ldots 2$

2.1 Revision A electrode array . . . . . . . . . . . . . . . 17

2.2 Revision B electrode array . . . . . . . . . . . . . . . . . . . 19

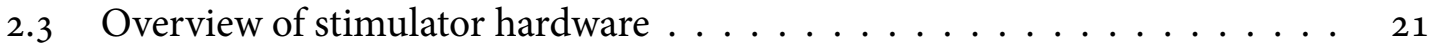

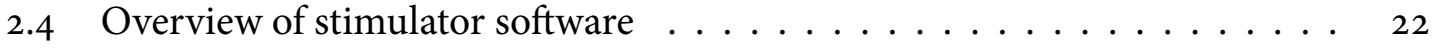

2.5 The monkey avatar $\ldots \ldots \ldots \ldots \ldots \ldots \ldots \ldots$

3.1 Schematic of the ICMS cue paradigm $\ldots \ldots \ldots \ldots \ldots \ldots \ldots \ldots$

3.2 Implant sites for the cue experiment $\ldots \ldots \ldots \ldots \ldots \ldots$

3.3 Receptive fields and stimulus waveforms $\ldots \ldots \ldots \ldots \ldots \ldots$

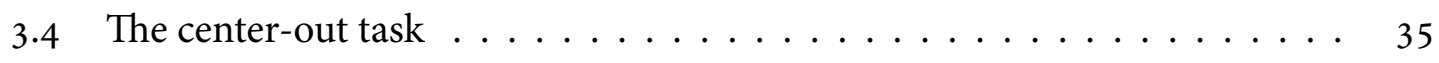

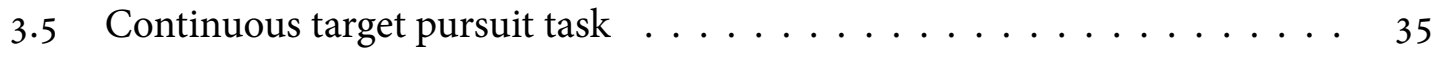

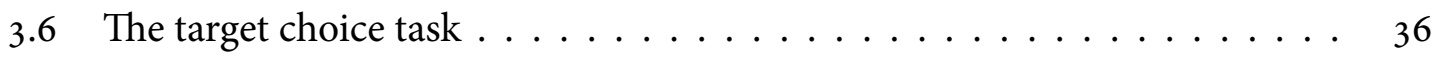

3.7 Examples of neuronal waveforms simultaneously recorded in three cortical regions .............................. 40

3.8 Example population directional tuning curves $\ldots \ldots \ldots \ldots \ldots$. . . . 41

3.9 Learning to use vibrotactile cueing . . . . . . . . . . . . . 43

3.10 Learning to use ICMS cueing. . . . . . . . . . . . . . . . 44

3.11 Learning after an ICMS-cue-response reversal . . . . . . . . . . . . 45

3.12 Psychometric curve for current amplitude . . . . . . . . . . . . 46

3.13 PETHs of M1 activity during ICMS cueing $\ldots \ldots \ldots \ldots \ldots$ 
3.14 Linear discriminant analysis (LDA) predictions of reach direction . . . . 49

3.15 LDA predictions of reach direction for hand control and brain control . . 50

3.16 Hand and brain control movements of the cursor with the vibrotactile cues 51

3.17 Hand and brain controlled movements of the cursor with ICMS cues . . 52

3.18 Average movements made during hand and brain controlled operations . . 53

4.1 The brain-machine-brain paradigm ............... 60

4.2 Implants for ICMS feedback experiment . . . . . . . . . . . 65

4.3 ICMS feedback stimulation paradigm $\ldots \ldots \ldots 6$

4.4 ICMS feedback task structure $\ldots \ldots \ldots \ldots \ldots \ldots$

4.5 ICMS patterns delivered for the artificial textures $\ldots \ldots \ldots \ldots 7$

4.6 Example of active reach with concurrent ICMS feedback . . . . . . . . . 68

4.7 Learning to use ICMS feedback . . . . . . . . . . . . . . . . . . . . 69

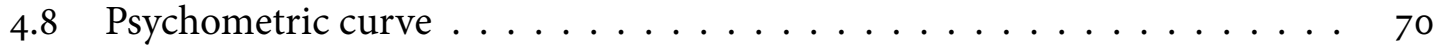

4.9 Monkeys rapidly learned vibration feedback $\ldots \ldots \ldots \ldots \ldots$

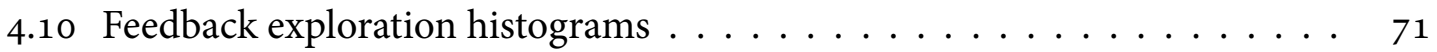

4.11 Exploration probabilities as function of reach number. $\ldots \ldots \ldots \ldots$

4.12 Control improves within sessions in hand and brain control . . . . . . . 74

4.13 M1 modulations during movement to different objects were not disrupted by concurrent ICMS . . . . . . . . . . . . . . . 76

4.14 Tuning adapts during brain control with ICMS feedback $\ldots \ldots \ldots \ldots$

4.15 Grouping of preferred directions occurs in $\mathrm{BCWOH} \ldots \ldots . \ldots 79$

4.16 Passive observation of the avatar elicits Mi firing rate modulations . . . . 80

4.17 Passively observed avatar movements can be predicted from M1 activity . . 81

5.1 Aperiodic task structure $\ldots \ldots \ldots \ldots \ldots$

5.2 Aperiodic ICMS stimulation paradigm $\ldots \ldots \ldots \ldots$

5.3 Example aperiodic ICMS pulse trains and distributions . . . . . . . 90 
5.4 Schematic of the blanking procedure . . . . . . . . . . . . 92

5.5 Learning to discriminate regular and irregular ICMS pulse trains . . . . . 94

5.6 Psychometric curves for different coefficients of variation $\ldots \ldots \ldots$

5.7 Time spent exploring the virtual objects as a function of CV . . . . . 96

5.8 Exploration of blanking intervals $\ldots \ldots \ldots \ldots \ldots$

6.1 Representing tactile gratings with ICMS patterns $\ldots \ldots \ldots \ldots$. . . . . 111

6.2 Spatiotemporal patterns of ICMS for representing texture $\ldots \ldots \ldots \ldots$ 


\title{
List of Abbreviations and Symbols
}

\author{
1-D One-dimensional \\ 2-D Two-dimensional \\ 3-D Three-dimensional \\ AT Artificial Texture \\ BC Brain control \\ BCWH Brain control with hand \\ $\mathrm{BCWOH} \quad$ Brain control without hand \\ BMBI Brain-machine-brain interface \\ BMI Brain-machine interface \\ CC Correlation coefficient (Pearson's $R$ ) \\ CV Coefficient of variation \\ DOF Degree of freedom \\ EMG Electromyographic recording \\ FES Functional electrical stimulation \\ HC Hand control \\ ICMS Intracortical microstimulation \\ ISI Interspike interval \\ LDA Linear discriminant analysis \\ M1 Primary motor cortex \\ NAT Null artificial texture
}




$\begin{aligned} \text { PD } & \text { Preferred direction } \\ \text { PV } & \text { Population vector } \\ \text { PETH } & \text { Perievent time histogram } \\ \text { PMd } & \text { Dorsal premotor cortex } \\ \text { PMv } & \text { Ventral premotor cortex } \\ \text { PPC } & \text { Posterior parietal cortex } \\ \text { RAT } & \text { Rewarded artificial texture } \\ \text { S1 } & \text { Primary somatosensory cortex } \\ \text { SMA } & \text { Supplementary motor area } \\ \text { SNR } & \text { Signal-to-noise ratio } \\ \text { UAT } & \text { Unrewarded artificial texture } \\ \text { UKF } & \text { Unscented Kalman filter } \\ \text { VR } & \text { Virtual reality } \\ \text { WF } & \text { Wiener Filter }\end{aligned}$




\section{Acknowledgements}

First and foremost, I must begin by thanking my parents, without whose love and support I could not have even contemplated going down this long and windy path.

Next, I acknowledge my original graduate supervisor, Craig Henriquez. I thank Craig for his enthusiastic support of my research interests over the years, despite the convoluted and lengthy trajectories that they have taken.

I thank my present graduate supervisor, Miguel Nicolelis, for taking on the risk of accepting a Biomedical Engineering graduate student into his lab. In doing so he gave me the incredible opportunity to conduct the research described in this document, for which I will be forever grateful.

I sincerely thank my committee: Craig Henriquez, Pat Wolf, Dennis Turner and Miguel for their support, guidance and patience. They have all been generous with their time and expertise and I am grateful for all of my interactions with them. I also thank Michael Platt, who was on my committee until very nearly the bitter end, when scheduling problems conspired to reduce the size of my committee to four.

I owe Misha Lebedev an enormous debt of gratitude. Misha has been a mentor and a friend at every step of the way and has been instrumental in training me in all aspects of systems neurobiology, experimental electrophysiology, and even monkey psychology. I thank him for teaching me all the tricks of the trade and giving me an appreciation for both the air and the bread of science.

I was fortunate to have excellent role models, colleagues, and friends in my fellow 
graduate students and post-docs in the lab. I especially thank those that I worked most closely with on this research: Nathan Fitzsimmons, Tim Hanson, Zheng Li, Katie Zhuang, Peter Ifft, and Solaiman Shokur. But I would be remiss to not also mention Jose Carmena, Andrew Tate, Eric Thomson, Shih-Chieh Lin, Romulo Fuentes, Hachi Manzur, Je Hi An, Jesse Winans, Andy Fuller, Manu Raghavan David Schwarz, and Leo Medina.

The Nicolelis laboratory has a tremendously helpful support staff. Susan Halkiotis, Gayle Wood, Terry Jones have all been extremely helpful with administrative matters. Gary Lehew and Jim Meloy provided me with invaluable engineering support. Laura Oliveira, Tamara Phillips, Weiying Drake, Kristen Dewey and Erin Carmena assisted me on countless matters of animal husbandry. None of the work described here could have been accomplished without the superb surgical assistance of Drgan Dimitrov.

I thank Max Hodak, Ian Peikon, Ben Grant, John Choi, Eric Bramley and all of the other undergraduates who have crossed research paths with me during my time at Duke for their assistance and insight.

Invaluable to my mental sanity were the friendships I developed among my fellow graduate students and postdoctoral colleagues in Biomedical Engineering, Neurobiology and other departments at Duke. At the risk of omitting many worthy friends, I here single out John Finan, Ken Eaton, Kevin Sampson, Sarah Roberts, John and Ann Wambaugh, George Hugh, Debbie Won and Chuck Dorval.

I also thank everyone in \#BoR.

Finally, for Lorrie Bradley I save my warmest thanks. I thank her for her superb editorial and proof-reading skills. I thank her for listening when I complained. I thank her for sharing in the excitement of discovery. I thank her for her love and support.

The work in this dissertation has been supported supported, at various times, by DARPA awards N66001-02-C-8022 and N66001-06-C-2019, NSF grant NSF-IBN-99-80043, NIH grant RC1HDo6339o and USAMRMC award W81XWH-08-2-0119. I was twice supported by the Duke University graduate school (in 2008 and 2010) with conference travel fellowships. 
In 2010 I also received a workshop travel award from the IEEE Engineering in Medicine and Biology Society, and a graduate student extended abstract award from the Biomedical Engineering Society. 


\section{1}

\section{Introduction}

$I^{N}$

$\mathrm{N}$ this dissertation I describe the development of a brain-machine-brain interface (BMBI;

Nicolelis \& Lebedev, 2009). A BMBI is a device that records efferent signals from the brain to measure internal states, computes a function of the recorded brain signals and measurements of external (non-brain) states, and stimulates the brain with the result of the computation to enact afferent influence on other brain states and the environment. A conceptual diagram of this idea is shown in Figure 1.1 on the following page.

Here, I consider a specific type of BMBI, one that uses efferent cortical brain signals to control a prosthetic actuator and provides afferent tactile somatosensory feedback about the interactions of the actuator and the environment. The development of this BMBI proceeded in three phases. First, I developed a simplified case where the efferent and afferent modules were very loosely coupled temporally (with a time constant on the order of seconds). Under this paradigm, rhesus monkeys (Macaca mulatta) made brain-controlled reaches based on intracortical microstimulation (ICMS) cues. Next, I increased the coupling between the afferent and efferent modules (decreasing the time constant to $100 \mathrm{~ms}$ ), which allowed rhesus monkeys to make reaches towards sets of virtual objects with a brain-controlled avatar, probe 


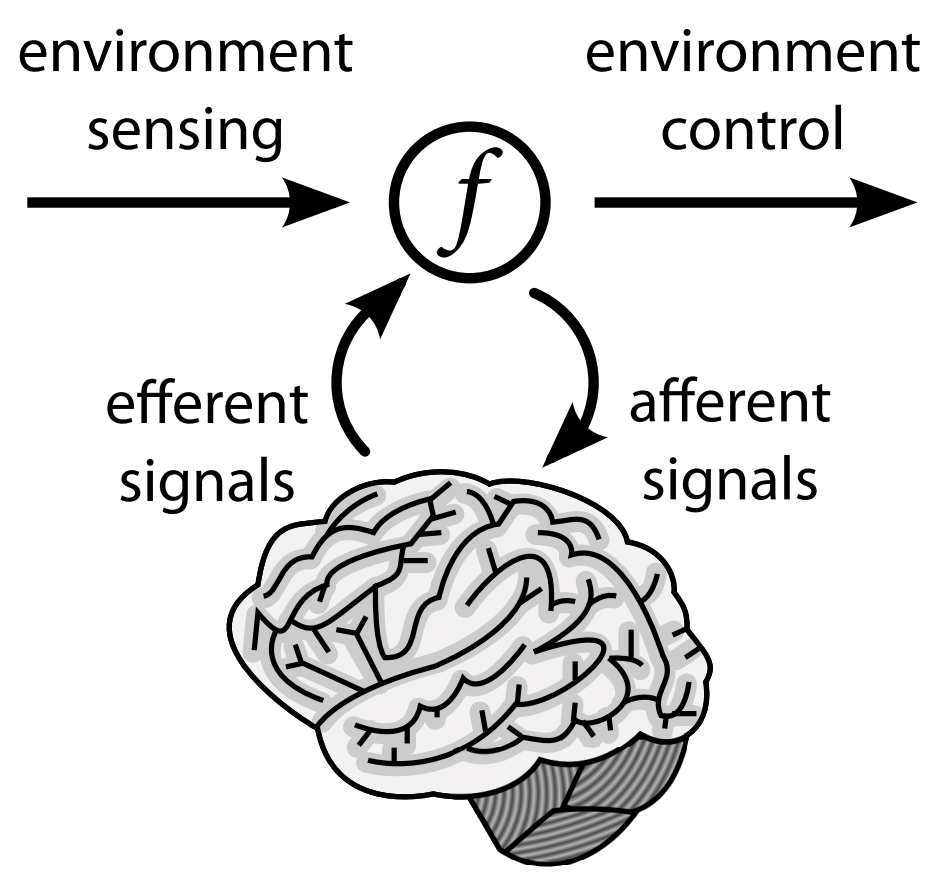

FIGURE 1.1: Schematic of the brain-machine-brain interface concept. The device performs a computation, $f$, based on efferent signals recorded from the brain that provide an estimate of internal states and the state of the environment. It exerts afferent control over other brain states and the environment.

their artificial textures using active touch, and select objects with particular textures. Finally, I probed the sensitivity of the primate somatosensory system to precise temporal patterns of ICMS using aperiodic pulse trains and determined an upper bounds on the bandwidth of artificial texture information that can be delivered to the primate somatosensory cortex using temporal encodings alone.

The antecedents of the work in this dissertation are described in Section 1.1. Section 1.2 gives a description of the remaining chapters.

\subsection{Review of previous work}

Despite their long and occasionally commingled histories, motor and sensory neuroprostheses (sometimes called neural prostheses) have developed mostly separately and have rarely 
been combined, although recently interest has been increasing (Nicolelis \& Lebedev, 2009).

Below, I briefly recount a historical perspective of the development of central nervous system (CNS) motor neuroprostheses and cortical sensory neuroprostheses. For the sake of brevity, I have restricted my review to neuroprostheses that extract motor commands directly from the activity of single neurons and populations of neurons, despite there being a significant literature on other, more noninvasive, approaches (Kübler et al., 2001; Birbaumer, 2006).

I take the same approach in a review of CNS sensory neuroprostheses that spans all sensory modalities. This is followed by a review of particular techniques for prosthetic somatosensation, some of which that do not necessarily rely on CNS stimulation. I close with a brief discussion of recently developed or proposed bidirectional neuroprosthesesdevices that both record and stimulate the nervous system.

\subsubsection{CNS motor neuroprosthesis}

\section{Operant conditioning}

The modern field of motor neuroprosthetics can trace its origins to the seminal studies of Eberhard Fetz (1969). Inspired by an earlier report (Basmajian, 1963) that human subjects could control the contraction of individual motor units with the aid of auditory and visual biofeedback, Fetz wondered if the activity of single pre-central cortical neurons (in what we would now call the motor cortex) could be voluntarily modulated. By providing auditory and visual feedback signals proportional to the cell activity, he was able train rhesus monkeys to increase the firing rates of single motor cortical cells by up to $500 \%$. In a series of subsequent experiments, Fetz and colleagues explored the operant conditioning of single cortical cells, weighted combinations of cortical neuron and muscle activation (including an interesting condition where muscle activity was required to be suppressed while cortical activity was increased), and even epileptiform activity (Fetz \& Finocchio, 1971; Fetz \& Wyler, 1973; Fetz \& Baker, 1973; Fetz \& Finocchio, 1975). 


\section{Early cortical motor neuroprostheses}

At about the same time that Fetz published his revolutionary findings, the idea of using the activity of cortical neurons to directly control the movements of external devices was starting to enter the consciousness of the research community. As Karl Frank, an early director of the Neural Interfaces Program at the National Institutes of Neurological Disorders and Stroke, prophesied, "We will be engaged in the development of principles and techniques by which information from the nervous system can be used to control external devices such as prosthetic devices, communications equipment, teleoperators ... and ultimately perhaps even computers" (Frank, 1968).

It was in this context that Edward Schmidt and his colleagues demonstrated that rhesus monkeys could modulate the activity of motor cortical neurons to precise levels (up to 8 different targets), achieving communication rates of almost 4 bit/s (Schmidt et al., 1977, 1978). There were three main innovations of Schmidt's work. First, Schmidt and colleagues explicitly discussed the possibility of using brain activity to control "devices external to the body, such as artificial limbs or neuromuscular stimulators for paraplegics" (Schmidt et al., 1978). Second, Schmidt and colleagues used chronically implanted electrodes. This allowed them to create a long-term connection with the nervous system, a requirement for any future clinical therapy and a preview of future research directions. Finally, the monkeys controlled the position of an LED-cursor with the activity of individual and combined motor cortical cells, again foreshadowing subsequent research.

\section{Directional tuning and the population vector}

The next big breakthrough occurred a few years subsequently, in the form of a discovery and an innovation from the laboratory of Apostolos Georgopoulos and colleagues. Georgopoulos discovered that the discharge rate of neurons in motor cortex were related not simply to the contraction of muscles, as had previously been observed by Evarts (1968), but could be understood and modeled as as a function of the direction of a reach, a property they 
called directional tuning (Georgopoulos et al., 1982). Each directionally tuned neuron had a preferred direction - a direction of movement corresponding with the maximal discharge rate. At angles farther and farther away from the preferred one, such tuned neurons fired less and less. Georgopoulos and colleagues modeled the firing of these neurons with cosine tuning functions.

The technical innovation came a few years later, when Georgopoulos and colleagues realized that by combining the activity of multiple neurons in a very simple way, they could accurately reconstruct the reaches of rhesus monkeys in three-dimensions (3-D; Georgopoulos et al., 1986). The algorithm that they devised was a simple vectorial sum of the contributions of each neuron along the axis of its preferred direction, an algorithm that they called the population vector (PV). Although the prosthetic applications of the PV were not immediately appreciated by Georgopoulos, the demonstration of population tuning nonetheless revolutionized ideas about how movement information is encoded in the brain.

Georgopoulos's population tuning hypothesis suggested that by recording simultaneously from populations of motor neurons, very accurate 3-D predictions of movements could be achieved. However, at the time it was unusual for researchers to record from more than a single neuron at time. Georgopoulos himself constructed his PVs from serial recordings of neurons. The prevalence of single electrode recordings persisted for nearly twenty years after the description of the PV, despite the fabrication and sporadic use of electrode array technology since the early 1970 (Wise et al., 1970; Chorover \& DeLuca, 1972). It was only after multielectrode array technology started gaining acceptance that the cortical neural prosthetic field started to make progress again.

\section{The dawn of the brain-machine interface}

In the late 1990s, after a series of investigations of the rodent somatosensory system with chronic multielectrode arrays (Nicolelis et al., 1993a,b; Nicolelis \& Chapin, 1994; Nicolelis et al., 1995), Miguel Nicolelis and John Chapin proposed that the use of chronic multielectrode 
arrays might enable neuroprosthetic applications (Nicolelis et al., 1997). Soon after, Chapin and colleagues demonstrated that populations of neurons simultaneously recorded in the primary motor cortex and ventrolateral thalamus of rodents could be used to control the 1-D movement of a robot arm (Chapin et al., 1999).

This demonstration was followed few years later by the work of Wessberg et al. (2000), who showed that the cortical activity of ensembles of precentral and postcentral neurons, recorded from the brains of owl monkeys (Aotus trivirgatus) while they reached towards food, could direct the 3-D movements of robotic arms locally and over the internet. While the monkeys did not observe the movements of the brain-controlled robots, this study was was seminal because it showed that the cosine tuning model (Georgopoulos et al., 1982) and population vector hypothesis (Georgopoulos et al., 1986), both of which had only been used with serial single neuron recordings previously, could be extended to multi-electrode array technologies and used in real-time to do useful work.

The progression of neuroprosthetic devices then rapidly accelerated. Serruya et al. (2002) next demonstrated that rhesus monkeys could rapidly control the movements of a 2-D cursor on a screen under visual feedback and without necessarily making any overt hand or arm movements (they only recorded from small ensembles of neurons, however). Later that year, Taylor et al. (2002) demonstrated closed-loop control of a computer cursor in 3-D using a coadaptive algorithm. Finally, Carmena et al. (2003) showed that rhesus monkeys could learn to control the reaching and grasping movements of a robot arm directly with ensembles of cortical neurons and, for the first time, documented evidence of the plastic changes in cortex that accompanied learning.

\section{Recent progress}

Since then there has been a veritable explosion of BMIs from numerous research laboratories. I enumerate a few of the most notable developments here. Musallam et al. (2004) developed what they called a "cognitive" prosthesis that used information from parietal cortex to control 
a BMI with movement intentions but not explicit trajectory signals. They also were able to simultaneously decode the value of perceived reward quality and quantity.

Velliste et al. (2008) demonstrated real-time control of an anthropomorphic multi-joint robotic arm for embodied interaction with the environment. Rhesus monkeys used the robotic arm for self feeding, making reaches in $3-\mathrm{D}$, and opening and closing the aperture of a gripper. It should be noted that the monkeys in this study controlled only the end-point of the robotic arm; robotic control algorithms handled the details of joint positioning.

Moritz et al. (2008), of Fetz's research group, demonstrated cortical control of functional electrical stimulation of arm muscles in pigtail macaques (Macaca nemestrina). The monkeys were able to use the neural interface and continue to move their arms even after temporary paralysis induced by temporary local anesthetic block (Pohlmeyer et al., 2009) of peripheral nerves. This research created the first direct artificial connection between the cortical brain cells and muscles, and demonstrated that neuroprosthetic devices can compensate for interrupted neural pathways and restore volitional control of the body.

The BMI devices described above used cortical activity recorded in upper-limb motor cortical representation areas. Fitzsimmons et al. (2009) showed, demonstrated that motor cortical activity could be used to enact prosthetic locomotion. The 3-D coordinates of leg joints and leg muscle electromyograms (EMGs) were reconstructed offline and in real-time. Additionally, high level kinematic parameters, such as step length, step frequency and speed were successfully decoded. Finally, decoded kinematic information was sent in real-time over the internet to an advanced bipedal robot in Japan, which reproduced the walking patterns (Cheng et al., 2007; Nathan Fitzsimmons, personal communication).

\section{Cortical BMIs in humans}

The above sections describe the development of cortical BMIs exclusively in animal models. There has however been some success in translating cortical BMIs into the clinic. This research has, naturally enough, tended to lag behind the animal experiments by several 
years. Below, I briefly discuss the most prominent studies, limiting my review to invasive approaches. Non-invasive BMIs have of course also developed rapidly and independently since the first demonstration by Farwell \& Donchin (1988). See Kübler et al. (2001) and Birbaumer (2006) for a review.

Kennedy \& Bakay (1998) provided the first evidence that human subjects could voluntarily modulate the activity of single cortical neurons. They implanted a "locked-in" patient suffering from amyotrophic lateral sclerosis with a "neurotrophic electrode" - an electrode that attracted the growth of neurons to its recording surface. The patient was able to learn to communicate by modulating the activity of the recorded neurons and maintained control for several months.

Shoham et al. (2001) examined five human subjects with spinal-cord damage using functional magnetic resonance imaging as they imagined executing the movements of their limbs. The subjects were found to have largely normal motor and somatosensory activation with respect to a cohort of intact control subjects. This study provided crucial evidence that patients with paralysis would retain the ability to voluntarily modulate their motor cortices, giving hope that the activity in these brain regions could be used to control a clinical cortical neuroprosthesis.

Patil et al. (2004) intraoperatively recorded the activity of neurons in the subthalamic nucleus and thalamic motor areas of 11 patients receiving deep brain stimulation electrodes. The subjects performed a hand grasping task under visual feedback. Offline analysis indicated that these subcortical sites could be use to predict hand grip force.

Leuthardt et al. (2004) measured electrocorticographic (ECoG) activity in four patients with temporary subdural electrode arrays implanted for the monitoring of epilepsy. ECoGs are an intriguing technology because they are less invasive than penetrating brain implants, but the communication bandwidth of ECoGs is not known. In this study, ECoG signals related to different types of motor and speech imagery were found to be voluntarily modulated and were used to move move a 1-D computer cursor in an online, closed-loop mode. 
Hochberg et al. (2006) implanted a patient with tetraplegia with a chronic electrode array in M1 as part of a pilot clinical trial. Activity recorded from populations of recorded neurons was used to directly control the movements of a cursor in closed-loop experiments. This study has provided the best evidence to date that the cortical neuroprosthesis techniques pioneered in animal studies can be directly translated to the clinic. Still, the patients enrolled in this study were eventually explanted, indicating that there remain numerous technical challenges to solve (Polikov et al., 2005).

Recently, Guenther et al. (2009) implanted a single "locked-in" patient with a neurotrophic electrode in a speech-related cortical region of the left precentral gyrus. The subject was able to learn to modulate activity on this electrode to control a speech synthesizer and produce four vowel sounds. This task was conceptually equivalent to 2-D cursor control along a space described by the frequencies of two formants and underscores that the neural decoding techniques used for arm reaching have broad application to other clinical therapies that have no simple animal model (i.e., speech production).

\subsubsection{CNS sensory neuroprosthesis}

Early electrical stimulation of the nervous system

It has been known that electric currents exert effects on the nervous system almost as long as there has been knowledge of electricity itself. Benjamin Franklin was one of the first to scientifically investigate the role that electricity could play in motor rehabilitation (Franklin, 1757). Luigi Galvani reported a few years subsequently that electric current could make the muscles of a recently deceased frog twitch (Galvani, 1791, translated in Green, 1953). Unfortunately, the views of the German physiologist Albrecht von Haller, who considered the cortex to be "insensitive", dominated the views of the era, so it took nearly a century before there was any serious investigation into applying electricity to the cortex (for a discussion see Neuburger, 1981). 


\section{Electrical stimulation of cortex}

Ignoring the prevailing dogma of the time, Gustav Fritsch and Eduard Hitzig discovered that electrical stimulation could evoke movement when applied to the motor cortex of dogs (Fritsch \& Hitzig, 1870, translated in von Bonin, 1960). Fritsch and Hitzig's work is especially notable because it conclusively demonstrated that the cerebral cortex did, in fact, serve an important neural function and that it was topographically organized. Sir Charles Sherrington subsequently used electrical stimulation to localize the organization of the baboon motor cortex (Brown \& Sherrington, 1911), suggesting that these same brain organization principles might also apply to man. Cushing (1909) was the first to observe that stimulation of cortex produced sensation in man. He stimulated the postcentral gyrus of two conscious patients and reported that the sensation was localized to body areas that were analogous to the part of the body that would have moved if the precentral gyrus was stimulated. Foerster (1936) replicated and extended these findings.

The next breakthrough came from Wilder Penfield, who who was a student of both Sherrington and Cushing. Penfield further extended the previous human cortical stimulation studies by systematically exploring the entire cortex of man (Penfield \& Boldrey, 1937; Penfield \& Rasmussen, 1952). He made the remarkable discovery that the size of a cortical representation area was proportional to the sensitivity of that part of the body. Essentially he described a map of the body found on the surface of the brain, but a distorted one. Penfield called this map a "homunculus" and described the existence of both motor and sensory homunculi.

\subsubsection{Sensory neuroprosthesis}

These results confirmed that stimulation of various parts of the nervous system could evoke subjective perceptual experiences. It was not long after that physicians and engineers started to investigate using stimulation of the nervous system for restoring sensation. There are two principal sensory modalities that have been explored to date: vision and audition. 


\section{Audition}

One of the first neuroprosthetic attempts in humans was the work of André Djourno and Charles Eyriès, who in 1957 stimulated the cochlear nerve of a patient who described experiencing auditory sensations (Eisen, 2003). This study, and numerous ones that followed using more advanced an elaborate multichannel stimulators (Merzenich et al., 1974; Loeb et al., 1983), eventually led to the development of the most successful neuroprosthesis to date, the cochlear implant. To date there has remained high interest in prosthetic hearing with ongoing investigations of auditory brainstem (Edgerton et al., 1982) and cortical (Deliano et al., 2009) auditory prostheses.

Vision

Foerster and colleagues (1936) were among the first to report that stimulation of the occipital lobe produced visual sensations, a finding that was expanded upon in by Penfield \& Rasmussen (1952). Brindley \& Lewin (1968) systematically explored the phosphenes experienced by a blind patient when stimulated at different locations and with different patterns. Dobelle et al. $(1974,1976)$ achieved the first visual prosthesis by coupling multichannel electrical stimulation of visual cortex with a camera. There has been considerable interest in visual prostheses since then, however most efforts have focused on optic nerve (Obeid et al., 2010) or retina-based (Ahuja et al., 2011) approaches.

\subsubsection{Prosthetic somatosensation}

Ways of creating prosthetic somatosensation have been much less explored, however, interest has been increasing in recent years. Much of this delay has been due to the relatively mysterious nature of somatosensation compared to the other senses. For many years there has been disagreement over the number and nature of the skin mechanoreceptors, of what unitary sensations they convey, and of exactly how those unitary sensations are combined into touch or proprioception (Kruger, 1996). 
Despite the lack of consensus in the periphery, it has known for the past 10 years that electrical microstimulation of the area $3 \mathrm{~b}$ subdivision of the somatosensory cortex of rhesus monkeys produces sensations that are, apparently, similar to the perception of flutter (Romo et al., 1998, 2000; de Lafuente \& Romo, 2005). Arguably the first prosthetic application of this discovery was the "robo-rat" technology developed by Chapin and colleagues to instruct the movements of rats (Talwar et al., 2002). Subsequently, Nathan Fitzsimmons and colleagues showed that owl monkeys can discriminate precise spatiotemporal patterns of microstimulation applied to primary somatosensory cortex (Fitzsimmons et al., 2007).

Since then interest in prosthetic somatosensation has dramatically increased (Fagg et al., 2007). London and colleagues demonstrated that rhesus monkeys can be cued by patterns of ICMS applied to the area 3a subdivision of primary somatosensory cortex, an area that is generally believed to be involved in the processing of proprioception and kinesthesia. Marasco and colleagues (2009) have used a targeted peripheral-nerve innervation technique to provide amputees with sensory substitution. Recently, Heming et al. (2010) explored the possibility that thalamic stimulation could be used for prosthetic somatosensation. They found a largely somatotopic organization and a variety of different somatosensory percepts, but patients reported that the evoked perceptions were generally "unnatural".

\subsubsection{Bidirectional neuroprosthesis}

One intriguing possibility for neuroprostheses is the possibility of bidirectional operation. Such a neuroprosthesis would sense brain activity and respond dynamically to keep the activity within a certain range or to provide the appropriate sensory feedback or motor response. One could consider dynamic patch clamp (Dorval et al., 2007) to be a very simple bidirectional interface with nervous tissue. However, there has been increasing interest in recent years in in vivo bidirectional interfaces.

One of the first proposed was the prosthetic hippocampus. (Berger et al., 2005; Song

et al., 2009). This would require a bidirectional link with the brain so that memories could 
be both laid down as well as retrieved. There have been no in vivo hippocampal prosthetic devices developed to date.

Bidirectional interfacing also makes a lot of intuitive sense for epilepsy control (Fountas \& Smith, 2007) and deep brain stimulation (Santaniello et al., 2011). For these therapies, closedloop bidirectional control would involve sensing when the nervous system was producing pathological activity (epileptic seizures or Parkinsonian tremor) and only stimulating at the minimum level and with the best timing for maximal therapeutic effect. Such devices could have significant power savings as well as other benefits for the patient.

Marzullo and colleagues have made some progress towards a closed-loop bidirectional location sense in a rodent model (Marzullo et al., 2010). The rats modulated single units in motor cortex in response to ICMS of primary visual cortex. Unfortunately, the authors were unable to demonstrate that the rats actually used the ICMS as feedback about task performance, only demonstrating that the rats used the ICMS to know when trials began.

Venkatraman and colleagues recently provided some evidence that rodents can use ICMS applied to barrel cortex to determine the location of virtual objects (Venkatraman \& Carmena, 2011). The rats made whisking motions and were microstimulated whenever their whiskers were above a rostral threshold or below a caudal threshold (depending on the trial). However, in their study the rats were rewarded for receiving microstimulation 4 to 6 times in a two second window, not make a discrimination. As such, the rats could have achieved reward by increasing the amplitude or frequency of whisking.

\subsection{Organization}

The work in the remaining chapters proceeds as follows. In Chapter 2 I describe in detail the experimental methods which are used in this dissertation. Methods are described in this chapter when they were common to two or more experiments. Otherwise they are detailed in the appropriate subsequent chapter. 
In Chapter 3, I describe experiments in which I combined an intracortical microstimulation (ICMS) cue, for the first time, with online brain-machine interface (BMI) for the decoding of reach. This experiment provides support for the hypothesis that that ICMS of S1 can form the basis of an afferent prosthetic input to the brain for guiding brain-controlled prostheses. I conceived the experiment with significant input from Mikhail Lebedev and Nathan Fitzsimmons. Animal training and neuronal recordings were conducted by Mikhail Lebedev and myself. The surgical team for array implantation was led by Dragan Dimitrov and Laura Oliveira. Timothy Hanson contributed to the design and manufacturing of the electrical stimulator. I was the primary contributor to the analyses. The experiments described in this chapter have been published (O’Doherty et al., 2009).

In Chapter 4, this approach is extended to an interleaved stimulation and recording paradigm that allows true somatosensory feedback mediated by ICMS concurrent with BMI-mediated reaches performed by an upper limb avatar. I conceived the experiment with with significant input from Mikhail Lebedev. Animal training and neuronal recordings were conducted by Mikhail Lebedev, Peter Ifft, Katie Zhuang and myself. The surgical team for array implantation was led by Dragan Dimitrov and Laura Oliveira. The monkey avatar was designed and programmed by Solaiman Shokur. I was the primary contributor to the analyses. The experiments described in this chapter are under peer review as of this writing.

Finally, in Chapter 5, I describe progress towards increasing the milieu of textures that can be represented by ICMS, by quantifying the range of parameters that can be discriminated by the monkey when periodic and aperiodic ICMS pulse trains are delivered. I conceived of the experiment. Animal training and neuronal recordings were conducted by Mikhail Lebedev and myself. The surgical team for array implantation was led by Dragan Dimitrov and Laura Oliveira. The monkey avatar was designed and programmed by Solaiman Shokur. Zheng Li conducted the artifact blanking analysis. I was the primary contributor all other analyses. The experiments described in this chapter are under peer review as of this writing. 


\section{Experimental methodology}

\subsection{Basic experimental procedures}

\subsubsection{Animal models}

Four adult rhesus macaque monkeys (Macaca mulatta) participated in this study: Monkeys C, G, M and N. Monkey C was male and weighed $6.5 \pm 0.6 \mathrm{~kg}$. Monkey G was male and weighed $6.7 \pm 0.5 \mathrm{~kg}$. Monkey M was male and weighed $8.2 \pm 0.7 \mathrm{~kg}$. Monkey N was female and weighed $6.4 \pm 0.4 \mathrm{~kg}$. All animal procedures were performed in accordance with the National Research Council's Guide for the Care and Use of Laboratory Animals and were approved by the Duke University Institutional Animal Care and Use Committee (IACUC).

\subsubsection{Basic behavioral preparation}

Each monkey was seated in a primate chair and viewed a computer display. In early experiments the display was large screen that used with an projector. In later experiments the display was a smaller LCD screen. The monkey's heads were left unrestrained and the recording system was connected to the implants using flexible wires. A two degree-of-freedom (left-right and forward-backwards) joystick was mounted at the monkey's waist level. The 
monkeys were trained to manipulate the joystick with their hands. Monkey G performed with the right hand; Monkeys $\mathrm{C}, \mathrm{M}$ and $\mathrm{N}$ performed with the left hand. A touch sensor on the joystick handle measured whether the monkey was holding the joystick. Monkeys $\mathrm{C}$ and G used a joystick with an electrical touch sensor; monkeys $\mathrm{M}$ and $\mathrm{N}$ used a joystick with an optical touch sensor. The optical touch sensor introduced less electrical noise into the recording system. Using the joystick, the monkeys controlled the movements of an actuator: either a circular cursor or an avatar arm. The cursor had a $1.6 \mathrm{~cm}$ diameter; the avatar is described below. Forward, backward, rightward, and leftward movements of the joystick translated to the upward, downward, rightward, and leftward movements of the actuator, respectively. The joystick to actuator gain varied between 3.2 and 6.4 depending on the session (i.e., a $1 \mathrm{~cm}$ movement of the joystick translated into a 3.2 to $6.4 \mathrm{~cm}$ movement of the cursor).

In some brain control sessions, brain control without hand movements (BCWOH), the joystick was removed. Of these BCWOH sessions, sometimes the monkey's arms were lightly restrained, however in many sessions there were no restraints on the movements of the monkey's arms.

\subsection{Cortical implants}

Each monkey received multiple microwire array implants in cortical areas, contralateral to their working hands. There were two electrode array designs that were used. In the text below I describe them as revision "A" and revision "B". Arrays built to the revision A design were implanted in monkeys $\mathrm{C}$ and $\mathrm{G}$; monkeys $\mathrm{M}$ and $\mathrm{N}$ received revision $\mathrm{B}$ arrays. All arrays were designed and manufactured in-house at the Duke Center for Neuroengineering.

\subsubsection{Revision A electrode array}

Revision A arrays consisted of 32 microwires grouped into 16 pairs. Each pair consisted of two microwires positioned tightly together. The array had a trapezoidal topology with two 


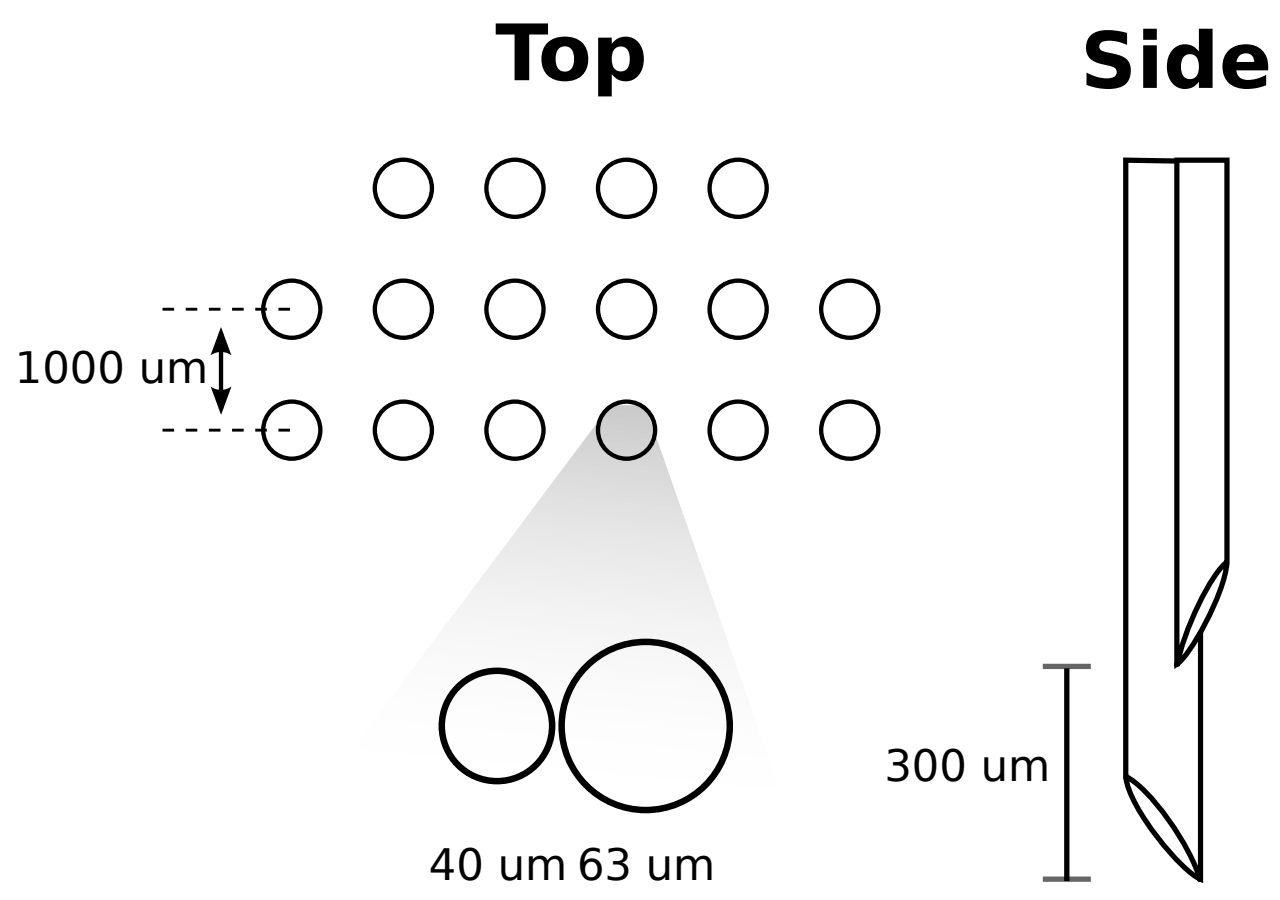

FIGURE 2.1: Revision A electrode array. Electrode diameters shown are examples.

rows of 6-pairs and a row of 4-pairs of electrodes. One electrode in each pair was $300 \mu \mathrm{m}$ longer than its mate, with the longer electrode always having equal or larger diameter. The separation between adjacent pairs was $1 \mathrm{~mm}$. Each electrode in a pair had its tips cut at an oblique angle. The arrays had heterogeneous electrode materials and diameters; see below for the details of specific arrays implanted in each monkey. For a diagram of the revision A array, see Figure 2.1.

\subsubsection{Revision B electrode array}

Each revision B electode array had 96 microwires, grouped into triplets. Within each array, the microwire triplets were grouped in two 4 -by-4 uniformly-spaced grids. The arrays were oriented such that one grid could be aligned over M1 and the other over S1. The grids were separated by $2 \mathrm{~mm}$, which could bridge the gap of the central sulcus. The separation between triplets of electrodes within each grid was $1 \mathrm{~mm}$. The electrodes of each triplet had three different lengths, staggered at $300 \mu \mathrm{m}$ intervals. The penetration depth of each triplet 
could be adjusted with a miniature screw. After adjustments during the month following the implantation surgery, the depth of the triplets was fixed for all recordings reported here. The longest electrodes in all triplets protruded to $2 \mathrm{~mm}$ in length measured from the cortical surface. The electrodes were all constructed of stainless steel 304. The insulation and electrode diameters in the array were heterogeneous, with each micro-wire coated in either polyimide or polytetrafluoroethylene and having a diameter of 50 or $63 \mu \mathrm{m}$. For a diagram of the revision B array design, see Figure 2.2 on the following page.

\subsubsection{Implant sites}

Monkey $\mathrm{C}$ received four revision A electrode arrays. Two arrays consisted of mixed tungsten and stainless steel 304 electrodes and were implanted in primary motor cortex (M1) and supplementary motor area (SMA). The tungsten electrodes were $50 \mu \mathrm{m}$ in diameter and coated with polyimide insulation; the stainless steel 304 electrodes were $45 \mu \mathrm{m}$ indiameter and coated with polytetrafluoroethylene. Monkey $\mathrm{C}$ also received two additional arrays that consisted of polyimide coated tungsten electrodes, all $50 \mu \mathrm{m}$ in diameter. These arrays were implanted in dorsal premotor cortex (PMd) and posterior parietal cortex (PPC).

Monkey $\mathrm{G}$ was implanted with three revision $\mathrm{A}$ arrays in the left hemisphere and three in the right hemisphere. Four with polyimide coated tungsten electrodes, all $50 \mu \mathrm{m}$ in diameter, were implanted in in M1 and PMd, bilaterally. Two arrays with polyimide coated stainless steel 304 electrodes with electrode pairs consisting of 40 and 63 micron diameters was implanted in S1, bilaterally. I only report on data from the three arrays implanted in the left hemisphere because the monkey used its right hand to move the joystick.

Monkeys $\mathrm{M}$ and $\mathrm{N}$ each received four 96-channel revision B arrays. Each received identical arrays implanted at the same implant sites. Each hemisphere received two arrays: one in the upper and one in the lower limb representation areas such that a single array corresponded to the sensorimotor cortical regions associated with a single limb. Each array sampled from neurons in both $\mathrm{M}_{1}$ and $\mathrm{S} 1$. In this study, I only report recordings from the 


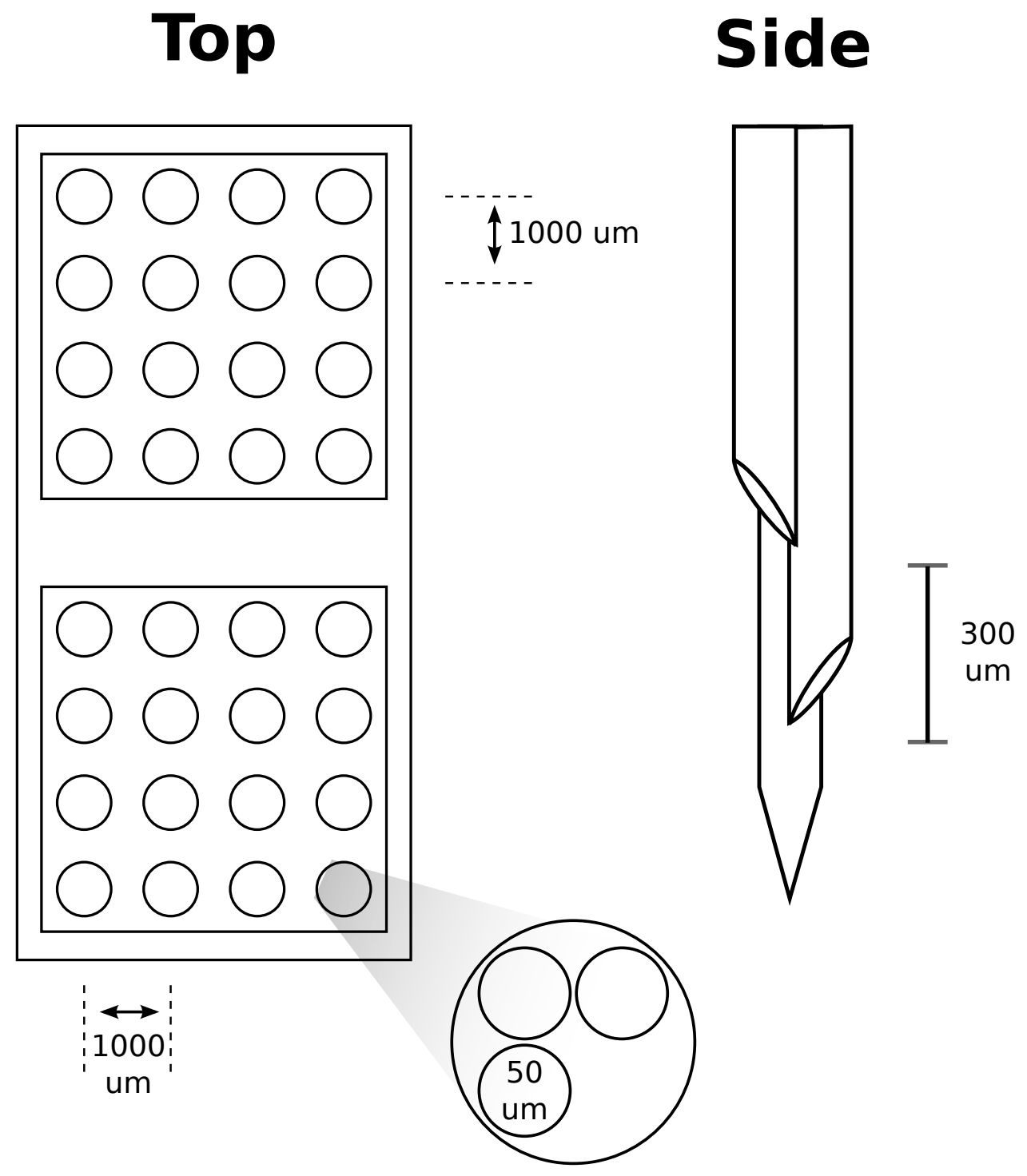

FIGURE 2.2: Revision B electrode array.

right hemisphere arm arrays in each monkey, since both manipulated the joystick with their left hands.

\subsection{Electrophysiological recordings}

Extracellular spiking activity was amplified, digitized, and high-pass filtered using Multichannel Acquisition Processors (Plexon, Inc.). Action potentials were detected and sorted 
on-line with templates set by the experimenter using Plexon spike-sorting software. The quality of the units was quantified by analyzing the refractory period, estimated from the interspike intervals (ISIs). To be qualified as a single unit, in addition to having a distinct shape and amplitude (Nicolelis et al., 2003), at least 99.9\% of its waveforms had to exhibit a refractory period greater than $1.6 \mathrm{~ms}$ (Hatsopoulos et al., 2004). Using these criteria 69.1\% of the recorded units were single units, and 30.9\% were classified as multi-unit neuronal activity. Single and multi-units were treated equally during online BMI sessions and for offline analysis purposes.

\subsection{Intracortical microstimulation}

The electrical microstimulator used in these studies was designed and constructed in-house. The stimulator was designed to be compact, reliable, flexible, low-noise and modularly expandable. This stimulator is high-side current controlled, which reduces stray capacitance between each isolated channel and animal ground. These currents must necessarily go through the stimulation electrodes, and ultimately through the ground that connects the animal to the wired recording systems. As such, it is desirable to minimize stray capacitance. The stimulator is highly flexible: The parameters of stimulation are all programatically adjustable by computer. Computer control permits stimulus trains of nearly arbitrary complexity to be enacted.

The the stimulation system is comprised of two principal components: stimulus isolation channels and a software interface.

\subsubsection{Stimulus isolation channels}

Four stimulator channels are assembled on a circuit board, providing independent and isolated monopolar or bipolar stimulation per electrode. Four of these boards can be stacked for a total of 15 stimulation channels controllable from a single computer. Each stimulation channel consists of an isolated power supply, digital-to-analog converter (DAC), bipolar 


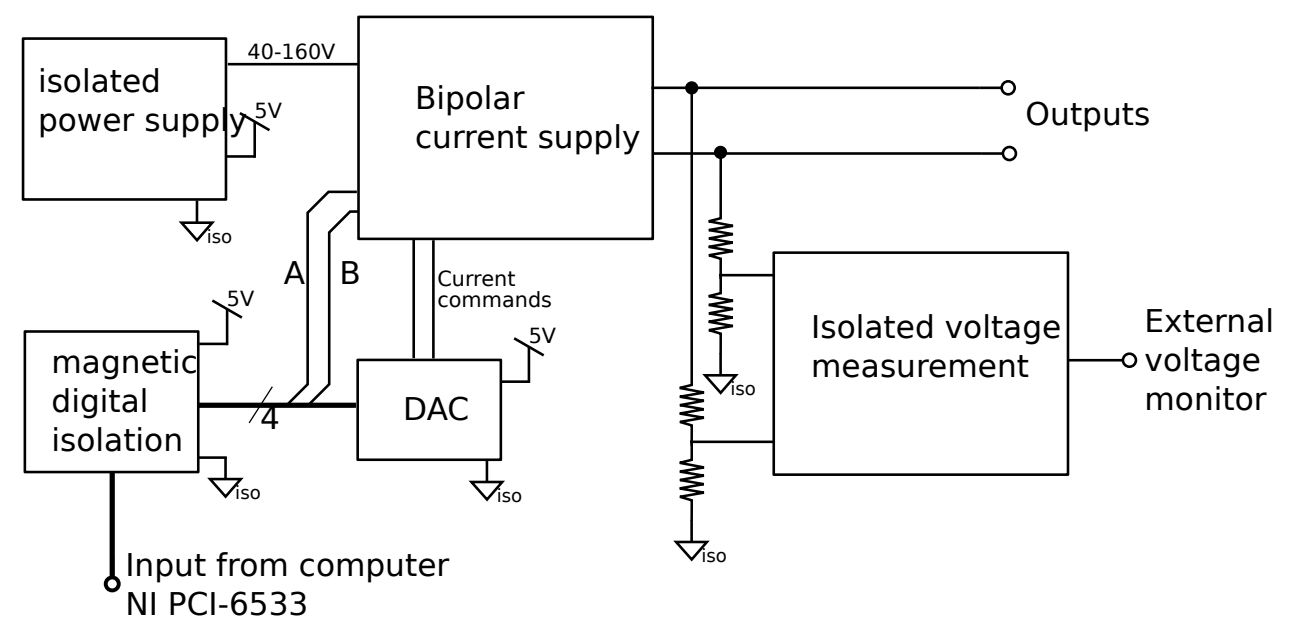

Figure 2.3: Overview of stimulator hardware. A single channel is shown.

current regulator, and an isolated monitoring circuit. The isolated power supply provides 40 to $160 \mathrm{~V}$ for driving high impedance electrodes. The core of the stimulation unit consists of the DAC and a voltage-feedback high-side current source. The isolated voltage measurement stage consists of an infrared LED and two matched photodiodes. Because the current command and resultant voltage are known, this circuit allows electrode resistance to be easily calculated. A diagram of the stimulator hardware is shown in Figure 2.3.

\subsubsection{Software interface}

The microstimulator is directly controlled via a kernel-mode driver program that provides direct memory access. The drivers operate the stimulator at $100 \mathrm{kHz}$, a rate fast enough to allow stimulation patterns with $10 \mu$ s precision. All parameters could be changed on the fly.

The primary attributes of a biphasic stimulation waveform are the amplitude and pulsewidth for an anodic and a cathodic current pulse, and intra-pulse interval. For normal operations the anodic amplitude and and pulse-width and intra-pulse interval are set, and the software automatically calculates the corresponding cathodic amplitude and pulse-width to balance the charge delivered. Asymmetric charge-balanced (or charge-unbalanced) waveforms are possible with the stimulation hardware, but were not used. Safety controls 


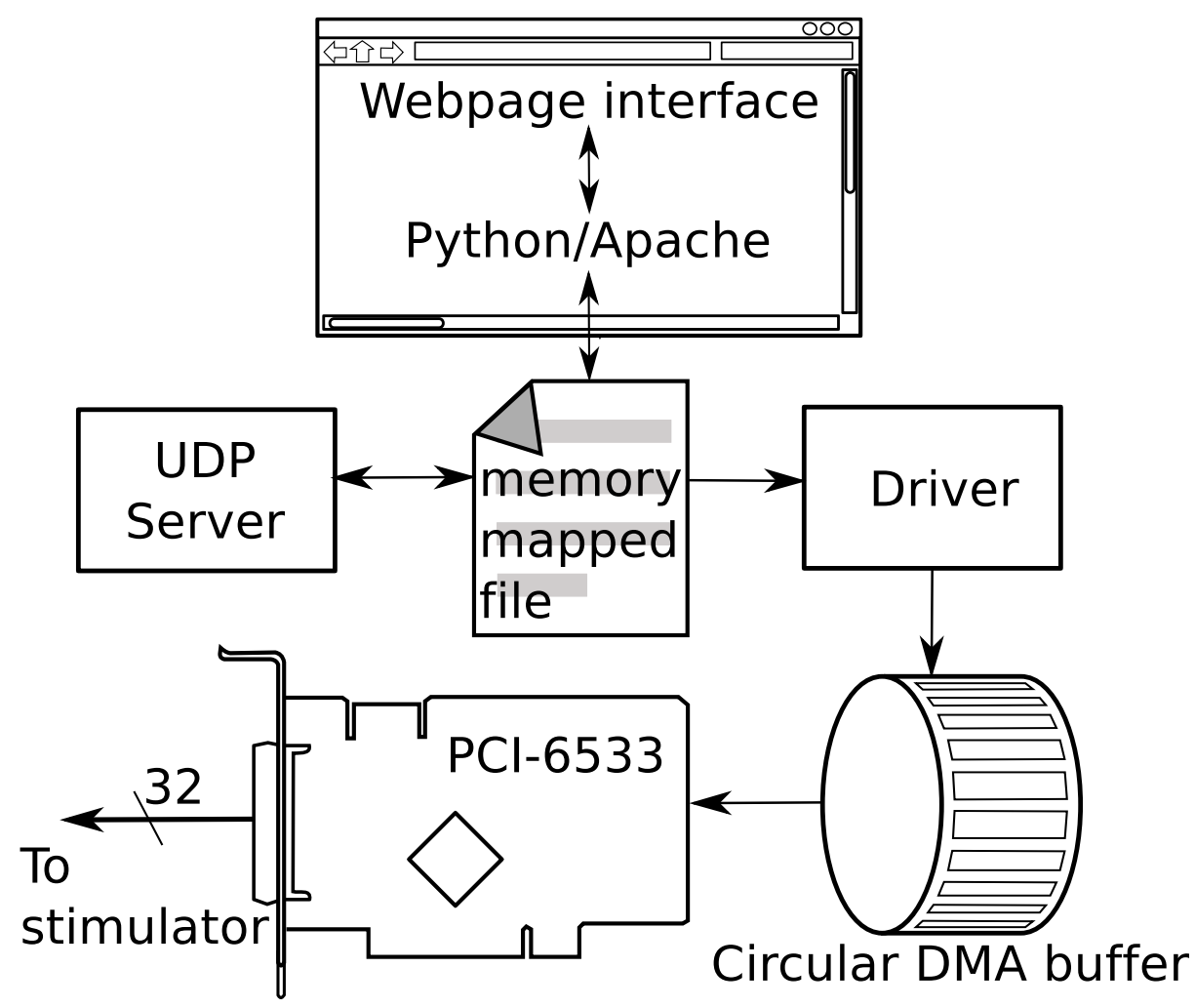

FIGURE 2.4: Overview of stimulator software.

in the software ensured that stimulation waveforms were always charge balanced, even if stimulation parameters were changed in the middle of a waveform. The stimulator can deliver arbitrarily complicated patterns of biphasic stimulation waveforms, but typically the most complicated was a second-order embedding (periodic packets of pulse-trains). The stimulator software can also deliver stochastic patterns of stimulation. These facilities were necessary for the experiments described in Chapter 5.

There were two interfaces provided to the microstimulator: a web interface and a UDP server. The web interface allowed parameters to be manually adjusted by a user via a convenient graphical interface. The web interface was used for debugging and to perform test stimulation runs, but was generally not used for most experiments. The UDP server provided an interface that our BMI software suite could programmatically interact with. 
This allowed for online automated control of stimulation parameters. A diagram of the stimulation software is shown in Figure 2.4 on the preceding page

\subsubsection{Psychometric measurements}

Psychometric measurements were conducted in several sessions for the experiments described in both Chapter 3 and Chapter 4 . These measurements were conducted to determine the minimum ICMS amplitude that the monkeys could discriminate. In these sessions, the ICMS amplitude was modulated on every trial. In each psychometric session a range of amplitudes was selected so that about half were in a range believed to be above the monkeys' threshold for discrimination and half were in a range of unknown discriminability. In this way, over several sessions, a large number of samples could be taken at each ICMS amplitude without frustrating the monkeys and negatively impacting behavior.

\subsection{Analysis methodology}

\subsubsection{Wiener filter}

For the experiments described in Chapter 3, cursor position was reconstructed using multiple Wiener filter (WF) linear decoding algorithms applied to the population of recorded neurons (Wessberg et al., 2000; Haykin, 2002; Carmena et al., 2003; Lebedev et al., 2005). The filter represented the $\mathrm{X}$ and $\mathrm{Y}$ coordinates of the joystick as a weighted sum of neuronal firing rates measured before the time of decoding:

$$
X(t)=b+\sum_{i=1}^{N} \sum_{j=1}^{M} w_{i j} n_{i}[t-(j-1) \tau]+\epsilon(t)
$$

where $X(t)$ is the value of the decoded parameter (for example, position of the joystick along the x-axis) at time $t, n_{i}$ is the firing rate of neuron $i, N$ is the total number of neurons, $M$ is the number of taps, $(j-1) \tau$ is the time delay for tap $j, w_{i j}$ is the weight for neuron $i$ at time tap $j, b$ is the $y$-intercept, and $\epsilon(t)$ is the residual error as a function of time $t$. The 
word tap here simply refers to a time delay between the input (neuronal firing rate) and output (joystick movement) of the filter. The value of the tap length parameter $\tau$ was set to $100 \mathrm{~ms}$, and the number of taps $M$ was set to 10. To put another way: the neuronal rates were sampled in a $1000 \mathrm{~ms}$ window preceding the time of decoding. The number of neurons $N$ varied with each recording session.

To calculate model weights, $w$, Equation 2.1 was converted into matrix form:

$$
\mathbf{X}=\mathbf{N W}+\epsilon,
$$

where $\mathbf{X}$ is the matrix of joystick movements, $\mathbf{N}$ is the matrix of neuronal rates, $\mathbf{W}$ contains the weights of the model and $\epsilon$ is the residual error. Each row of $\mathbf{N}$ corresponds to a specific time and each column is a vector of data for a particular neuron at a particular time lag. Since our models took into account ten lags, matrix $\mathbf{N}$ had ten columns for every neuron. The y-intercept was calculated by prependeding a column of ones to matrix N. Matrix $W$ was then found using ridge regression:

$$
\mathbf{W}=\left(\mathbf{N}^{T} \mathbf{N}+\lambda_{W} \mathbf{I}\right)^{-1} \mathbf{N}^{T} \mathbf{X}
$$

where $\lambda_{F}$ is the ridge regression parameter and $\mathbf{I}$ is the identity matrix.

\subsubsection{Unscented Kalman filter}

In the experiments described in Chapter 4 and Chapter 5, cortical activity was transformed into actuator movements using an Nth-order Unscented Kalman filter (UKF; Li et al., 2009) that has been shown to be superior to linear approaches for decoding arm movements. The filter used was up to a 1oth-order UKF in some sessions, but in most the 3 rd-order UKF was sufficient. The filter parameters were fit based on the hand movements of the monkeys while they performed the task using a joystick, or in some of the experiments described in Chapter 4 , based on passive observation of avatar movement while the monkeys' arms were restrained. 


\subsubsection{Cross validation}

Each filter was first trained (i.e., the values of weights $\mathbf{W}$ were calculated) and then used as the decoder for new data. Accordingly, each experimental record was split into two halves: the training set and the validation set. The model was trained on the first set of the experimental data and predictions were obtained using the second set. Decoding was also conducted for the reverse arrangement: training the models on the second set and using it to predict the first set.

\subsubsection{Performance metrics}

To measure the performance of BMI decoders, two metrics were employed: correlation coefficient (CC) and signal to noise ratio (SNR).

\section{Correlation coefficient}

Correlation coefficient, also known as Pearson's $R$ is the ratio between the covariance between two statistical variables and their standard deviations. Thus, when comparing a signal and a prediction of that signal the CC between them is defined as:

$$
\operatorname{CC}(X, \hat{X})=\frac{\operatorname{cov}(X, \hat{X})}{\sigma_{X} \sigma_{\hat{X}}}
$$

where $X$ is the known variable, $\hat{X}$ is the prediction of that variable, cov is the covariance, and $\sigma_{X}$ and $\sigma_{\hat{X}}$ are the standard deviations of $X$ and $\hat{X}$, respectively. The CC can vary in the range:

$$
-1 \leq \mathrm{CC} \leq 1
$$

with unity corresponding to the signals corresponding exactly, zero corresponding to a lack of correspondence, and negative values can correspond to an inverted relationship between the variables (for example between $\sin (x)$ and $\cos (x)$ ). Correlation coefficient (and 
sometimes squared correlation coefficient) are the most frequently used performance metric in the BMI field. However, CC has several disadvantages that make it tricky to interpret and to compare values between research groups. Therefore I also used an additional performance metric, the signal to noise ratio.

\section{Signal to noise ratio}

The signal to noise ratio (SNR) is a ratio between the power of the signal and the power of the noise, that is, the ratio of the squared amplitude of the signal to the squared amplitude of the noise:

$$
\operatorname{SNR}(X, \hat{X})=10 \cdot \log _{10}\left(\frac{\sigma_{X}^{2}}{\operatorname{MSE}(X, \hat{X})}\right)
$$

For purposes of measuring BMI performance, the signal is the known variable, $X$, and the noise is the difference between the known variable and the predicted variable, $X-\hat{X}$. The variance of a random variable is defined as:

$$
\sigma_{\theta}^{2} \equiv \mathbb{E}\left[\left(\theta-\mu_{\theta}\right)^{2}\right]
$$

where $\mathbf{E}$ is the expectation operator and $\mu_{\theta}$ is the mean of the variable.

MSE is the mean squared error between the known and predicted variables:

$$
\operatorname{MSE}(\theta, \hat{\theta}) \equiv \mathbb{E}\left[(\theta-\hat{\theta})^{2}\right]
$$

and is computed in an analogous way.

Therefore, $\sigma_{x}^{2}$, the variance of signal, can be interpreted as the power of the signal and $\operatorname{MSE}(X, \hat{X})$ as the power of the noise. The ratio of these two is the signal to noise ratio. It is expressed in decibels $(\mathrm{dB})$. 
Unlike CC which is bounded between positive and negative unity, SNR is unbounded and can take any value between positive and negative infinity. A SNR of zero means the signal and noise exist in equal proportions.

\subsection{Real-time operations}

\subsubsection{Data preprocessing}

Spikes were counted in $100 \mathrm{~ms}$ non-overlapping bins to estimate the instantaneous firing rate. Joystick position was recorded at $500 \mathrm{~Hz}$ and down-sampled to $10 \mathrm{~Hz}$ to match the binning rate. Velocity was calculated from position by two-point digital differentiation. An sensor in the joystick handle measured if the monkey was holding the joystick; intervals where the joystick was not held were disregarded for model training. Real-time processing was implemented on a custom software system $\left(\mathrm{BMI}_{3}\right)$ running on a workstation with an Intel Core i7 $9202.67 \mathrm{GHz}$ processor.

\subsubsection{Brain control}

For each of the tasks described in Chapter 3 and Chapter 4, online, closed-loop experiments were performed. The monkeys first performed the behavioral tasks manually using the handheld joystick (Hand Control; HC) and later controlled the cursor movements directly with their cortical activity. The behavioral and neural data, recorded during 10 to 30 min training epochs during which the monkeys moved the joystick manually, were used to train multiple decoding models (described above) that extracted cursor position from the modulations of cortical cells, as described above. Once the model weights were calculated, the mode of operation was switched to brain control during which the joystick was disconnected from the cursor, and cursor position was directly controlled by the signals extracted from the brain. For some sessions the joystick was immediately removed upon transition to brain control (Brain Control Without Hand movements; BCWOH); for other sessions, the joystick was disconnected from the task control but otherwise left alone and the monkey was permitted 

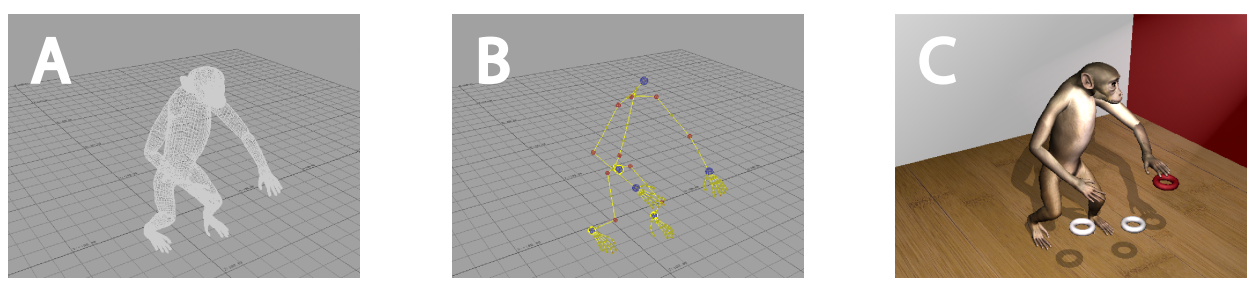

FIGURE 2.5: The monkey avatar. Here it is shown from a third-person perspective for illustration purposes. The the realism of the avatar was due to three subsystems: A. a realistic 3-D mesh, B. a skeletal model, C. realistic texturing and lighting.

to continue to move its hand (Brain Control With Hand movements; BCWH). For some $\mathrm{BCWOH}$ sessions the monkeys arms were gently restrained; for others they were permitted to move their arms if they chose to. Some experimental sessions, brain control consisted of both $\mathrm{BCWH}$ and $\mathrm{BCWOH}$ trial types.

\subsubsection{Monkey avatar}

In some experiments (see Chapter 4 and Chapter 5), the monkey controlled the movements of a virtual-reality (VR) monkey avatar with rich and realistic kinematic movements and spatial interactions. The avatar was designed and rigged with 3ds Max (Autodesk, Inc., San Rafael, CA) and animated in real-time using Motion Builder (Autodesk, Inc., San Rafael, CA). A custom plug-in extension for bidirectional communication with Motion Builder was implemented to control the avatar and receive tactile information from the virtual environment. The avatar control loop rate was $50 \mathrm{~Hz}$, with visual refreshing at $30 \mathrm{~Hz}$.

The avatar model was designed to depict a rhesus conspecific, and had 128 controlled degrees of freedom: 25 for each hand, four for each arm, three for each leg, 25 for each foot, six for the spine and the head, three for each eye (including eyebrows) and one for each ear. The avatar can additionally exhibit various facial expressions, random body movements and eye blinking in order to make its appearance more lifelike. 
In these experiments, the avatar was shown to the monkey from a first person view with only the upper limbs visible to the monkey. In the experiments described here, only the left upper-limb of the avatar was shown to the monkeys. Avatar posture was controlled using a mixture of direct control of end effectors and inverse kinematics. The physical interdependencies of the hand joints and a library of predefined hand postures were also implemented (Rijpkema \& Girard, 1991). 


\section{3 \\ Sensory cue}

\subsection{Abstract}

$\left\{\begin{array}{l}\text { RAIN-MACHINE INTERFACES (BMIs) establish channels of direct communication be- } \\ \text { tween the brain and artificial actuators. As such, they hold considerable promise for }\end{array}\right.$ restoring mobility and communication in patients suffering from severe body paralysis. To achieve this end, future BMIs must also provide a means for delivering sensory signals from the actuators back to the brain. Prosthetic sensation is needed so that neuroprostheses can be better perceived and controlled. Here we show that a direct intracortical input can be added to a BMI to instruct rhesus monkeys in choosing the direction of reaching movements generated by the BMI. Somatosensory instructions were provided to two monkeys operating the BMI using either: (A) vibrotactile stimulation of the monkey's hands or (B) multi-channel intracortical microstimulation (ICMS) delivered to the primary somatosensory cortex $\left(\mathrm{S}_{1}\right)$ in one monkey and posterior parietal cortex (PPC) in the other. Stimulus delivery was contingent on the position of the computer cursor: the monkey placed it in the center of the screen to receive machine-brain recursive input. After two weeks of training, the same level of proficiency in utilizing somatosensory information was achieved with ICMS of S1 as 
with the stimulus delivered to the hand skin. ICMS of PPC was not effective. These results indicate that direct, bidirectional communication between the brain and neuroprosthetic devices can be achieved through the combination of chronic multi-electrode recording and microstimulation of S1. We propose that in the future, bidirectional BMIs incorporating ICMS may become an effective paradigm for sensorizing neuroprosthetic devices.

\subsection{Introduction}

During the last decade, considerable progress has been made in research on neuroprosthesesdevices that attempt to treat neurological impairments and limb loss by interfacing intact neural structures with artificial devices that enact motor or sensory functions (Nicolelis, 2003; Lebedev \& Nicolelis, 2006; Schwartz et al., 2006; Fetz, 2007). Sensory neuroprostheses, such as cochlear (Merzenich et al., 1974; Fallon et al., 2008) and visual (Dobelle et al., 1974; Dagnelie, 2008) implants, make use of electrical stimulation of sensory neural structures to recreate afferent input and perceptual experiences. Motor neuroprosthetics, often called BMIs (Nicolelis, 2001), transform neuronal activity recorded in motor areas into commands to move artificial actuators such as cursors (Serruya et al., 2002; Taylor et al., 2002), and robotic devices (Wessberg et al., 2000; Carmena et al., 2003; Velliste et al., 2008), and for the functional stimulation of muscles (Moritz et al., 2008).

Curiously, sensory and motor neuroprosthetics have developed largely in parallel, without attempts to build integrated systems with both motor and sensory capabilities. Normal interactions with the environment, however, require conjoint processing of sensory and motor signals (Witney et al., 2004; Crapse \& Sommer, 2008). Thus, the neurophysiological mechanisms underlying voluntary motor control in mammals are critically dependent on the afferent innervation of the skin, muscles, and joints (Ribot-Ciscar et al., 2003; James et al., 2007). Indeed, the paucity of sensory signals available in current BMIs could be a factor limiting their future clinical usefulness. 


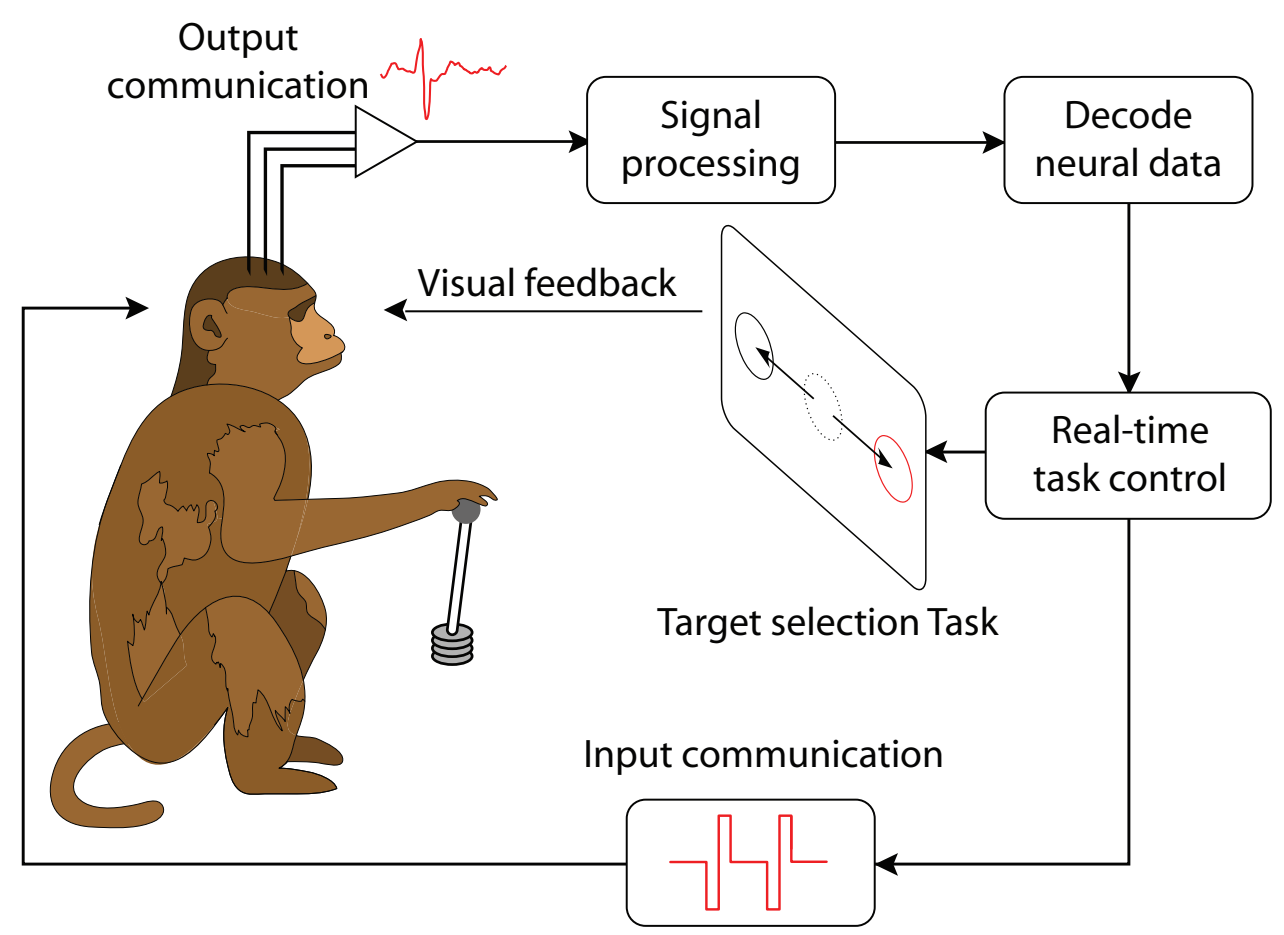

FIGURE 3.1: Schematic of the ICMS cue paradigm. A monkey is seated in front of a video screen to which the images of a cursor and visual targets are projected. The animal acquires targets with the cursor either manually using a joystick or directly by the activity of populations of motor-cortical cells. If more than one target is presented, reach direction is instructed by joystick vibration or artificial somatosensation delivered via ICMS of primary somatosensory cortex $\left(\mathrm{S}_{1}\right)$.

Here we propose that future clinical neuroprostheses could be based on a bidirectional system in which motor control signals are extracted from the brain, using multi-electrode arrays implanted in motor areas, while tactile, proprioceptive, and other useful signals are sent back to the brain through spatiotemporal patterns of intracortical microstimulation (ICMS) delivered to sensory areas (Lebedev \& Nicolelis, 2006). To advance this goal, we added a direct intracortical input to a BMI, based on multi- electrode cortical microstimulation used by a rhesus monkey for enacting arm reaching movements (Figure 3.1). This intracortical input instructed the direction of BMI-generated cursor movements. 

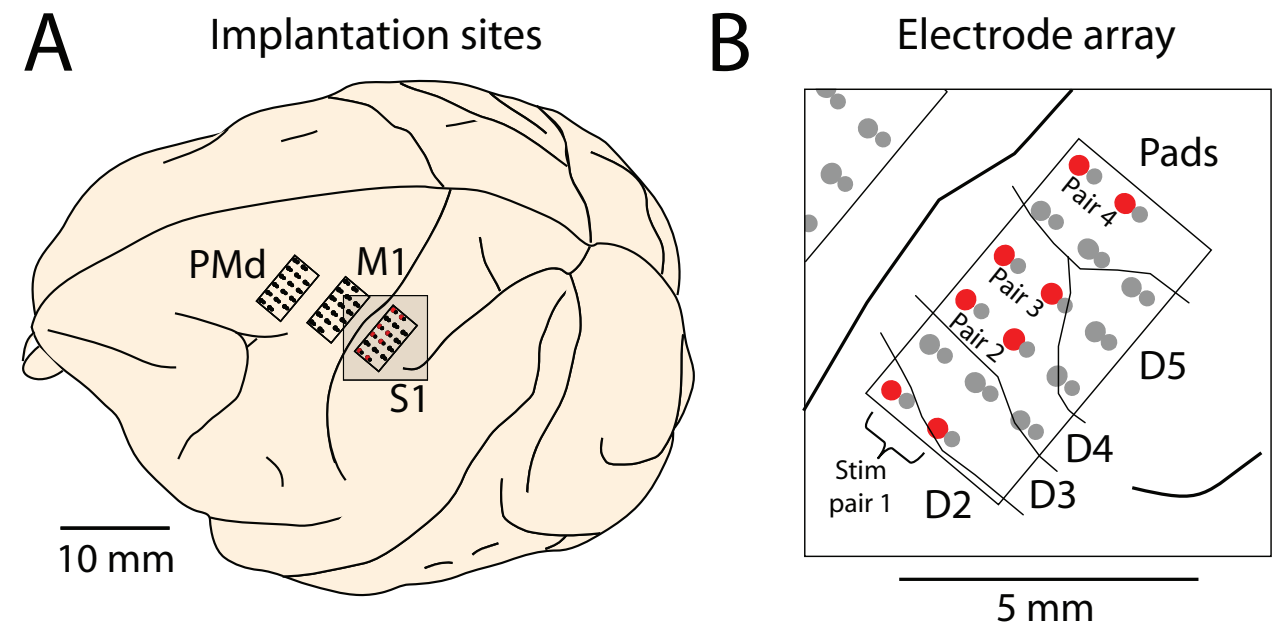

FIGURE 3.2: Implant sites for the cue experiment. A. Monkey G was implanted with microwire arrays in two motor-cortical areas (dorsal premotor cortex, PMd, and primary motor cortex, $\mathrm{M1}$ ) and a primary somatosensory area (S1). C. Zoomed-in region focuses on the S1 implant in monkey G. Red circles indicate electrode pairs used for ICMS. Lines between electrodes indicate functionally determined receptive field boundaries. The electrode grid covered the representation of digits $\mathrm{D}_{2}-\mathrm{D}_{5}$ and hand pads.

\subsection{Material and methods}

\subsubsection{Cortical implants}

Each monkey received multiple microwire array implants in cortical areas, contralateral to their working hands. Aseptic surgery was performed to implant electrode arrays. Monkey G was implanted with six microelectrode arrays (32 microwires in each; 3.2 ) in primary motor cortex (M1), primary somatosensory cortex $\left(\mathrm{S}_{1}\right)$, and dorsal premotor cortex $(\mathrm{PMd})$ of both hemispheres. For the present study, we report only data from the arrays in the left hemisphere of Monkey G. Monkey C was implanted with four 32-microwire arrays in the right hemisphere: M1, PMd, posterior parietal cortex (PPC), and supplementary motor area (SMA). Further details of the electrode arrays is contained in Section 2.2.

The PMd and M1 arrays were used to extract motor commands and the S1 array was employed as the target for ICMS (panel C of Figure 3.2). In the second animal, motor signals were extracted from the SMA, PMd, and M1 arrays, and ICMS was delivered to the PPC 

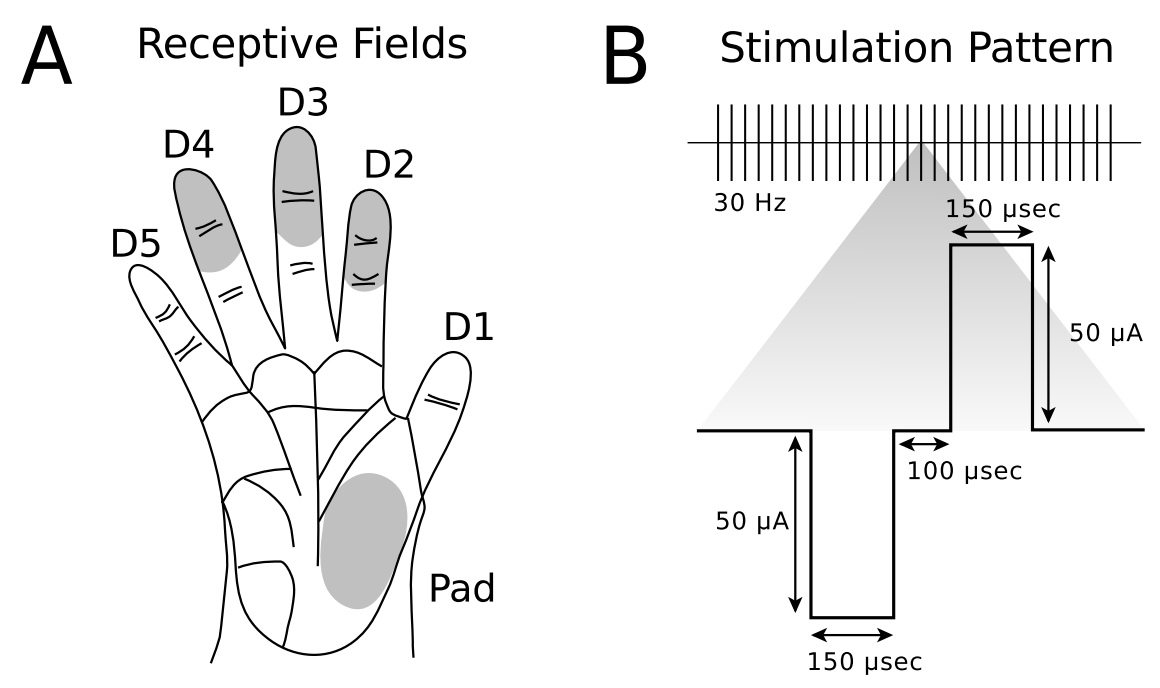

FIGURE 3.3: Receptive fields and stimulus waveforms. A. Illustration of the monkey palm with the shaded regions indicating functionally mapped receptive fields that were on the electrode pairs used for ICMS in monkey G. B. Typical parameters of an ICMS pulse train used in this study.

array. The electrodes chosen for ICMS in Monkey G yielded recorded S1 neurons with clear receptive fields located on the ventral aspect of the second, third, and fourth digits and palm pads (panel B of Figure 3.2 on the preceding page and Figure 3.3). No clear receptive fields were identified for the PPC implant in Monkey C. In this animal, we used the same arrangement of the stimulation electrodes within the electrode grid as described for Monkey G.

\subsubsection{Behavioral tasks}

Three behavioral tasks were employed. The first two tasks, center-out (Figure 3.4 on the following page) and continuous target pursuit (Figure 3.5 on the next page), served to estimate basic parameters of the BMI such as prediction accuracy and neuronal tuning to motor parameters. The third task, target choice (Figure 3.6 on page 36 ), incorporated somatosensory input. Each task required placing the computer cursor into a target using 


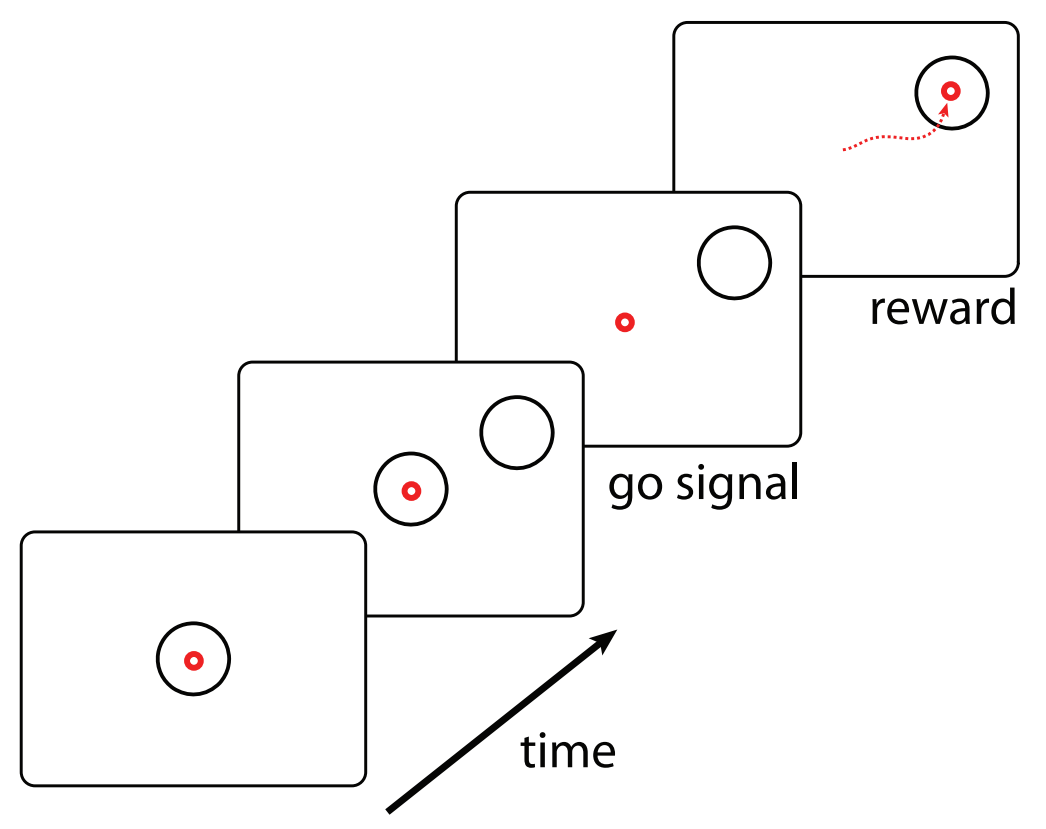

FIGURE 3.4: The center-out task. Monkeys first placed the cursor at a central location and then moved it to eccentrically located targets.

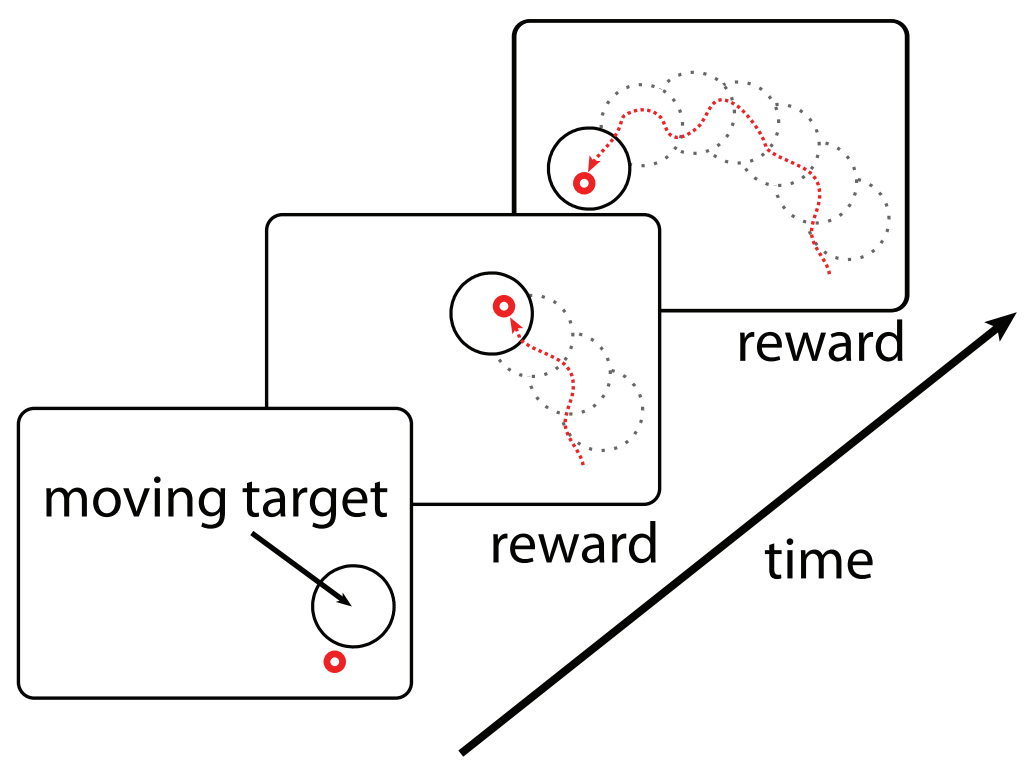

FIGURE 3.5: Continuous target pursuit task. Monkeys were rewarded for keeping the cursor within a continuously moving target. 


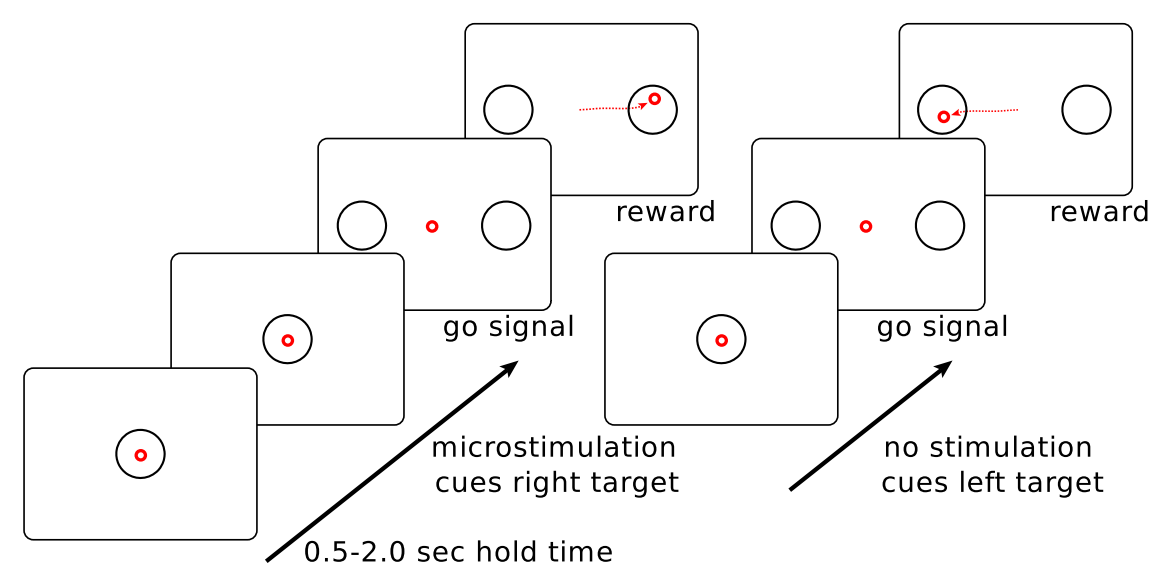

Figure 3.6: The target choice task. Monkeys selected one of two targets. Directional instruction was provided by joystick vibration or intracortical microstimulation (ICMS).

the joystick. Correct performance was rewarded by juice rewards. The center-out task used stationary targets that occurred at randomly chosen locations on a fixed-radius from the center of the screen. The monkey was required to hold the cursor at the central target and, once a peripheral target appeared, move the cursor to that target.

The pursuit task used a moving target that followed a Lissajous curve (Hales, 1945; Finkle, 1965) as described by:

$$
\begin{aligned}
& x=A \sin (a v t+\delta) \\
& y=B \sin (b v t),
\end{aligned}
$$

where $x$ and $y$ are the $\mathrm{x}$ - and $\mathrm{y}$-axis coordinates and $t$ is time in milliseconds. We used the following parameter values: 


$$
\begin{aligned}
& a=3 \\
& b=4 \\
& v \in\{0.00015,0.0002\} \mathrm{kHz} \\
& \delta=0.5 \pi \\
& A=B=22.4 \mathrm{~cm} .
\end{aligned}
$$

The target choice task started with the monkey centering the cursor at a target located in the middle of the screen. Then, an instruction period of 0.5 to $2 \mathrm{~s}$ followed. During this interval, a somatosensory stimulus was presented that instructed reach direction. In some experimental sessions, the stimulus instructed reaching to the right; in the others it instructed reaching to the left. If no stimulus was delivered, the monkey had to reach to the opposite direction. The stimulus itself was either a vibration of the joystick handle or ICMS. In some sessions, the stimulus was delivered for the entire instruction interval; in others, it was delivered for a fraction of the total instruction interval. After the instruction period ended, the central target disappeared and two choice targets appeared on the screen. The monkey was rewarded for responding by placing the cursor over the target (right or left) corresponding to the direction indicated by the stimulus delivered during the instruction interval.

In some sessions, we repeated the same stimulus in the trial immediately following one in which the monkey made an error. This was done for training purposes to prevent the monkeys from choosing the same target repeatedly without ever making reaches to the other target. However, when this rule was employed the monkeys could potentially make correct reaches in these trials without attending to the stimulus. Therefore, in sessions where this technique was employed, we restricted our analysis to trials that followed a rewarded trial and excluded those that followed non-rewarded trials. 


\subsubsection{Intracortical microstimulation}

Electrical stimulation was accomplished with a custom-built, 4-channel, current-controlled, optically isolated stimulator (see Subsection 2.4.1). Simulation trains used in this study consisted of symmetric, biphasic, charge-balanced waveforms delivered synchronously and in a bipolar fashion through adjacent pairs of electrodes. Current amplitudes of $25-60 \mu \mathrm{A}$ and pulse widths of $150-200 \mu$ s were used at frequencies between 30 and $60 \mathrm{~Hz}$, depending on the session (panel E of Figure 3.3 on page 34). The initial current amplitudes used for ICMS of $\mathrm{S}_{1}$ were determined by first finding the threshold current for evoked movements in M1 and then selecting a value just below that threshold. There was a $100 \mu$ s delay between the cathodic and anodic phases of the stimulation waveforms due to limitations of the stimulation hardware.

\subsubsection{Linear discriminant analysis}

To classify reach direction during the instruction period of the target choice task, we used linear discriminant analysis (LDA; Fisher, 1936). The interval from $1 \mathrm{~s}$ prior to the onset of the instruction period to $1 \mathrm{~s}$ after the offset was broken into $50 \mathrm{~ms}$ bins. The neuronal firing rate for each of the neurons in the ensemble was placed into a vector for each time-bin. This procedure was repeated for each trial. The trials were then partitioned into those in which the reach was made to the right and those where the reach was made to the left. From each of these sets, half of the trials were selected for training the classifier and half were selected for cross-validation. Using equal numbers of trials from each training set, linear classifiers were trained to discriminate reaches to the left and to the right for each time-bin. These classifiers, however, acted causally and cumulatively based on information accumulated from previous time-bins. This means that the classifier at time $t=T$ used the output of the

preceding classifiers $(t<T)$ as well as the firing rates of the neurons at time $t=T$ to classify reach direction. Performance of the classifier was measured with the cross-validation set. 


\subsection{Results}

In this study, a somatosensory component was added to our previous design of a BMI enacting arm reaching movements (Carmena et al., 2003). This component instructed the direction of reaching movements. Motor output of the BMI was controlled by the activity of neuronal ensembles recorded in the primary motor $\left(\mathrm{M}_{1}\right)$ and dorsal premotor $(\mathrm{PMd})$ cortex (panel B of Figure 3.2 on page 33). The behavioral tasks consisted of acquiring visual targets with a computer cursor (Figure 3.1 on page 32 and Figure 3.6 on page 36). Monkeys first performed the behavioral tasks manually using a hand-held joystick and then switched to controlling the cursor directly by their cortical activity. Predictions of cursor movement were generated using linear models (Wessberg et al., 2000; Carmena et al., 2003) Two types of somatosensory input were employed: (a) vibrotactile stimulation of the monkeys' palms via a vibrator embedded in the joystick handle and (b) ICMS of primary somatosensory ( $\left.\mathrm{S}_{1}\right)$ cortex in one monkey (panel E of Figure 3.3 on page 34) and the arm reach area of posterior parietal cortex (PPC) in the other monkey. Both monkeys learned to use vibrotactile stimulation cues to instruct the direction of their cursor movements. The first monkey learned manual and BMI tasks instructed by ICMS of S1; the other was unable to learn to use ICMS of PPC.

\subsubsection{Ensemble activity during reaching tasks}

We recorded simultaneously from ensembles of 50 to 200 cortical neurons, depending on the experimental session and subject (Figure 3.7 on the following page). Analysis of directional tuning indicated that the preferred directions of the recorded neurons covered the entire range of angles from 0 to $360^{\circ}$ (Figure 3.8 on page 41 ). Concurrent with recordings during behavioral tasks, we performed periodic mapping of receptive fields of the neurons recorded in S1. These neurons revealed receptive fields distributed primarily on the hand and digits (panel B of Figure 3.2 on page 33 and panel A of Figure 3.3 on page 34). 


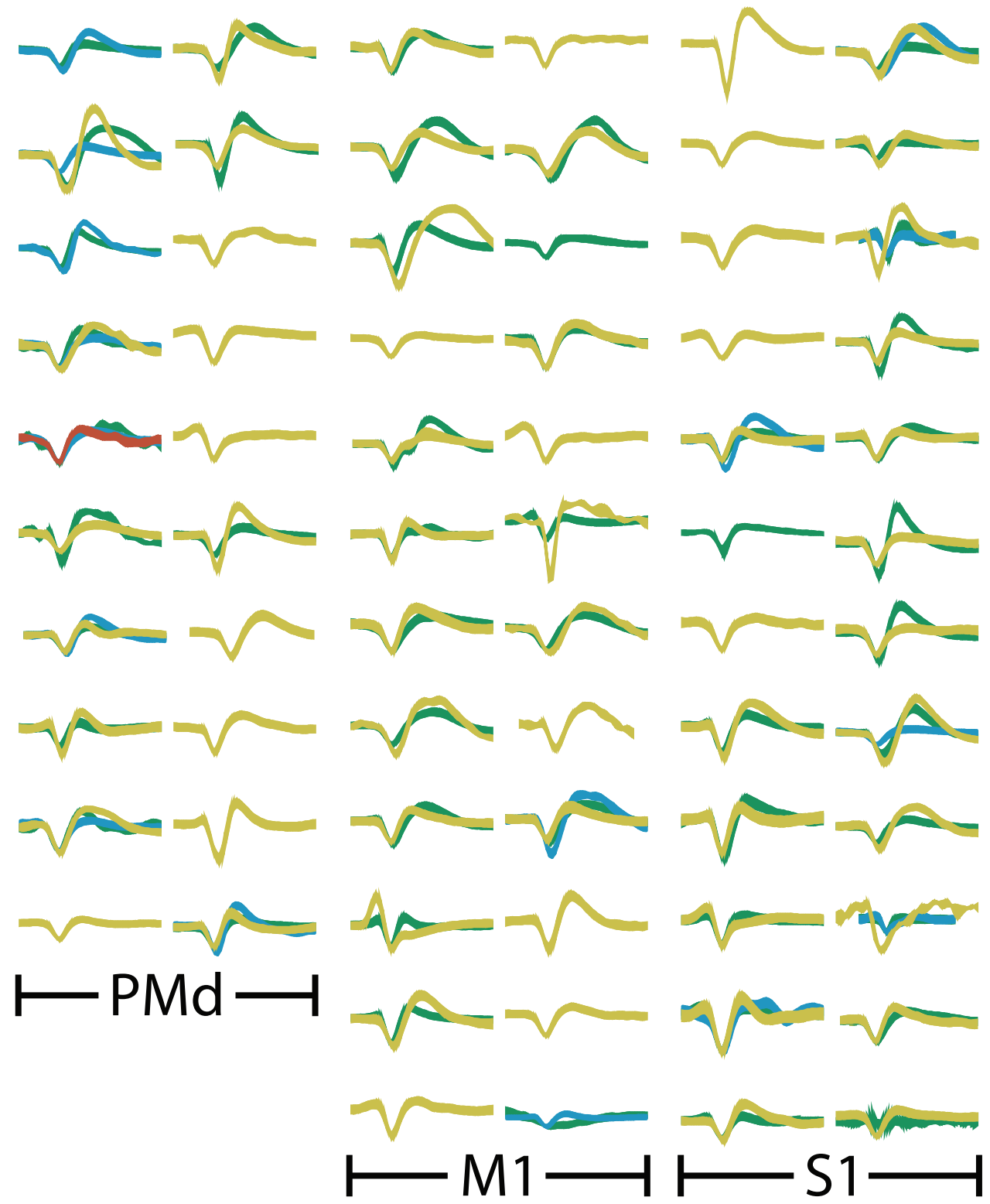

FIGURE 3.7: Examples of neuronal waveforms simultaneously recorded in three cortical regions (PMd, M1 and $\mathrm{S}_{1}$ ). Each colored shape represents the sorting template used to capture the firing of a single unit. Up to four neurons were sorted on a single channel and are represented by the colors yellow, green, blue, and red. 

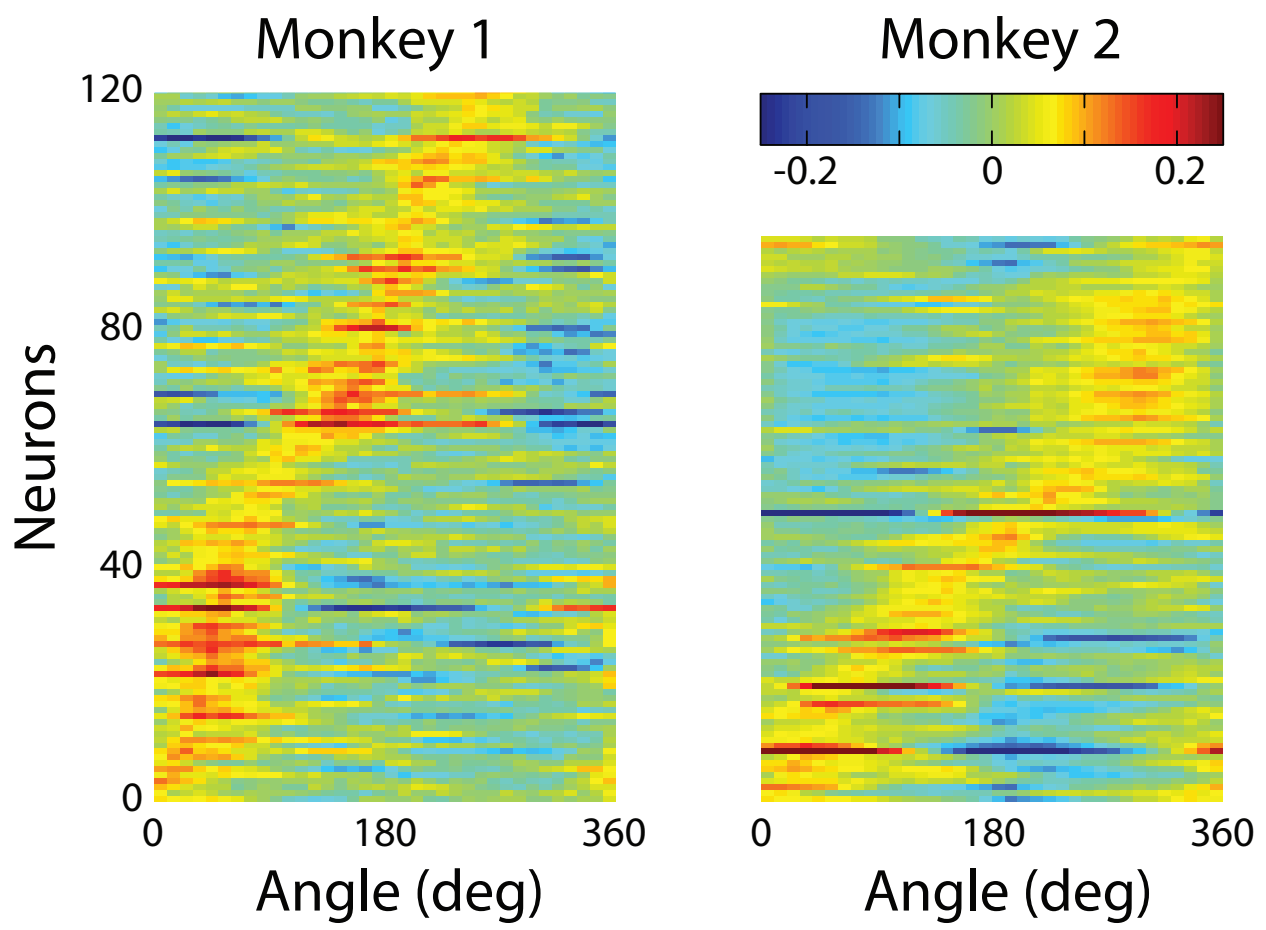

FIGURE 3.8: Example population directional tuning curves. Directional tuning curves for the ensembles of cortical neurons recorded in monkeys G (left) and C (right). Each horizontal line represents a neuron. Color represents firing rate normalized by standard deviation as a function of movement direction; neurons are sorted by preferred direction.

\subsubsection{Decoding movement}

The accuracy of predictions of hand position from cortical ensemble activity observed was in the same range as has been achieved in previous studies carried out in our laboratory (Wessberg et al., 2000) and by other groups (Taylor et al., 2002). The prediction accuracy was higher in the early recording sessions, when the majority of implanted electrodes yielded the most high quality units and later decreased as some of the channels lost unit activity. Table 3.1 shows the average performance of the predictions under different conditions. Across all tasks and sessions, when performing in HC mode, the average correlation coefficient (CC), between the actual and predicted values of the joystick X-position, was $0.47 \pm 0.19$ (mean \pm standard deviation) for monkey G and $0.53 \pm 0.14$ for monkey C. The CC for Y-position was $0.42 \pm 0.18$ and $0.51 \pm 0.17$ for monkeys $\mathrm{G}$ and $\mathrm{C}$, respectively. 
Table 3.1: Average prediction accuracy for the sensory cue task. Correlation coefficient between the actual hand movements and movements as predicted from cortical activity across different behavioral conditions for two monkeys. Sessions with brain control with hand movements $(\mathrm{BCWH})$ are shown split into the initial hand control portion, when the online filter was trained, and the subsequent brain control portion. The mean \pm standard deviation is presented.

\begin{tabular}{lll}
\hline Session Type & Monkey G & Monkey C \\
\hline \hline HC & N(sessions $)=139$ & $N=78$ \\
\hline$x$ & $0.47 \pm 0.19$ & $0.53 \pm 0.14$ \\
$y$ & $0.42 \pm 0.18$ & $0.51 \pm 0.17$ \\
\hline \hline BCWH & $N=20$ & $N=21$ \\
\hline Hand Control Portion & & \\
$x$ & $0.57 \pm 0.20$ & $0.58 \pm 0.14$ \\
$y$ & $0.60 \pm 0.20$ & $0.45 \pm 0.19$ \\
Brain Control Portion & & \\
$x$ & $0.41 \pm 0.19$ & $0.48 \pm 0.16$ \\
$y$ & $0.50 \pm 0.27$ & $0.38 \pm 0.21$ \\
\hline
\end{tabular}

The correspondence between the actual and predicted hand position decreased in sessions where the monkeys were permitted to continue to move their hands during direct brain control (BCWH; Table 3.1). The CC for X-position decreased 28.1\% $(P<0.01$ Wilcoxon signed-rank test) and $\mathbf{1 7 . 2 \%}$ in Monkey C $(P<0.001)$. The CC for Y-position decreased 16.7\% $(P<0.01)$ and $15.6 \%(P<0.005)$ in monkeys $\mathrm{G}$ and $\mathrm{C}$, respectively. This decrease indicates that the neuronal ensemble adapted to controlling the cursor movements and became less representative of the animal's hand movements-a finding that we and others have previously interpreted as evidence of the external actuator becoming incorporated into the brain's internal representation (Lebedev et al., 2005; Tkach et al., 2007).

\subsubsection{Somatosensory stimulus}

Incorporation of somatosensory input into the BMI was investigated in the target choice task (Figure 3.6 on page 36). The monkeys chose between two visually identical targets based on a somatosensory input (vibration or ICMS), the presence or absence of which instructed 


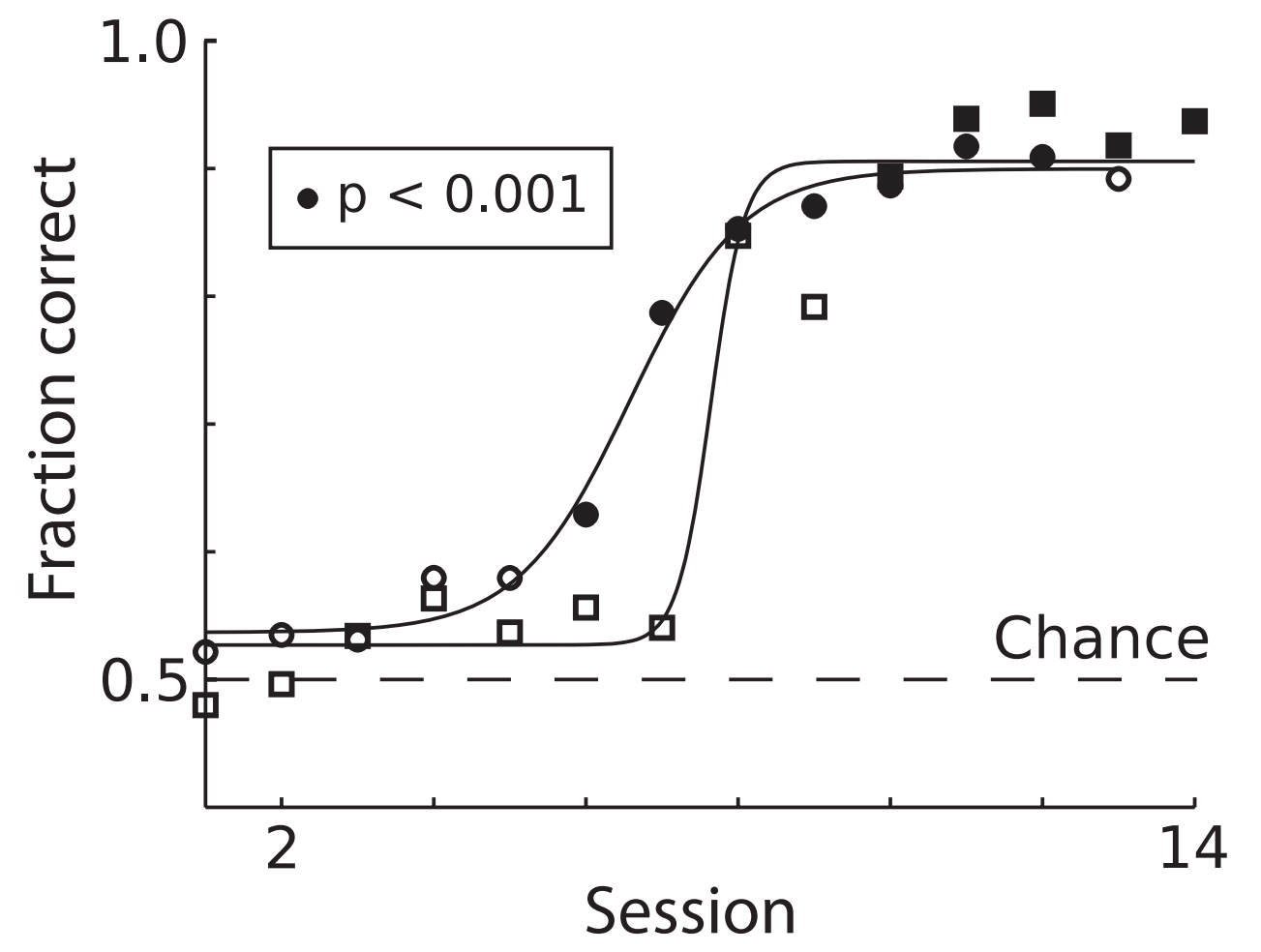

FigURE 3.9: Learning to use vibrotactile cueing. Circles indicate Monkey G; squares indicate Monkey C. Filled symbols indicate statistically significant difference from chance performance $(P<0.01)$, open symbols correspond to chance performance. Solid curves are the lines of best sigmoidal fit.

reach direction (right or left identical circles; Figure 3.6 on page 36). In initial experiments with Monkey G, the presence of a stimulus instructed reaches to the right, and the absence of stimulus instructed reaches to the left. This contingency was later reversed (see: Figure 3.11 on page 45 ). Monkey $\mathrm{C}$ always reached to the left if the stimulus was presented and reached to the right if it was absent.

Monkey $\mathrm{G}$ was initially trained with vibrotactile stimulation and was able to perform at $90 \%$ accuracy after 12 sessions (Figure 3.9). After 15 sessions of training in this task, the vibration cue was replaced with ICMS delivered to S1. However, the previous training with vibrotactile stimulation did not immediately transfer to the ICMS task. Rather, the monkey started with chance performance and began to improve only on the 15th session. After this 


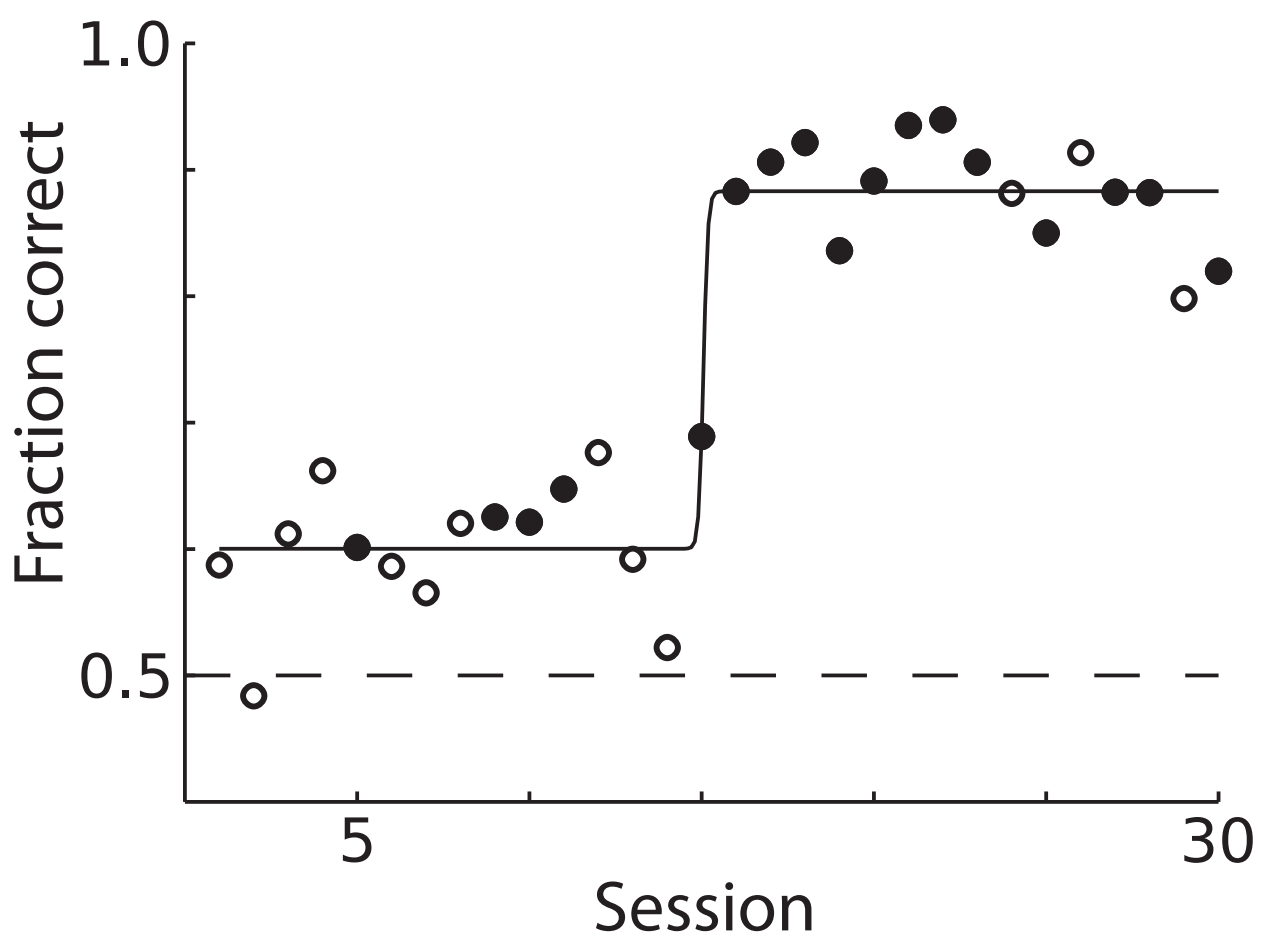

FIGURE 3.10: Learning to use ICMS cueing. Data is shown from Monkey G. Solid curve is the line of best sigmoidal fit.

point, learning was very rapid and the monkey achieved 90\% accuracy by the 20 th session. (Figure 3.10).

However, even before rapid behavioral improvement occurred in the ICMS task, the monkey's performance was marginally, but statistically, above chance (6o.02\% versus $52.5 \%$ in control trials without microstimulation; $P<0.001$, Wilcoxon ranked-sum test). This effect could reflect a bias in the monkey's reach decision that resulted from the activation of motor preparatory circuits in the stimulated hemisphere (Hanks et al., 2006). If so, this bias was not crucial for task performance because the monkey learned a reverse contingency in which the instructed target was ipsilateral to the stimulated hemisphere (Figure 3.11 on the next page).

Psychometric measurements in which ICMS current amplitude was varied on different trials showed that Monkey G could respond to currents as low as $25 \mu \mathrm{A}$ (with a pulse width 


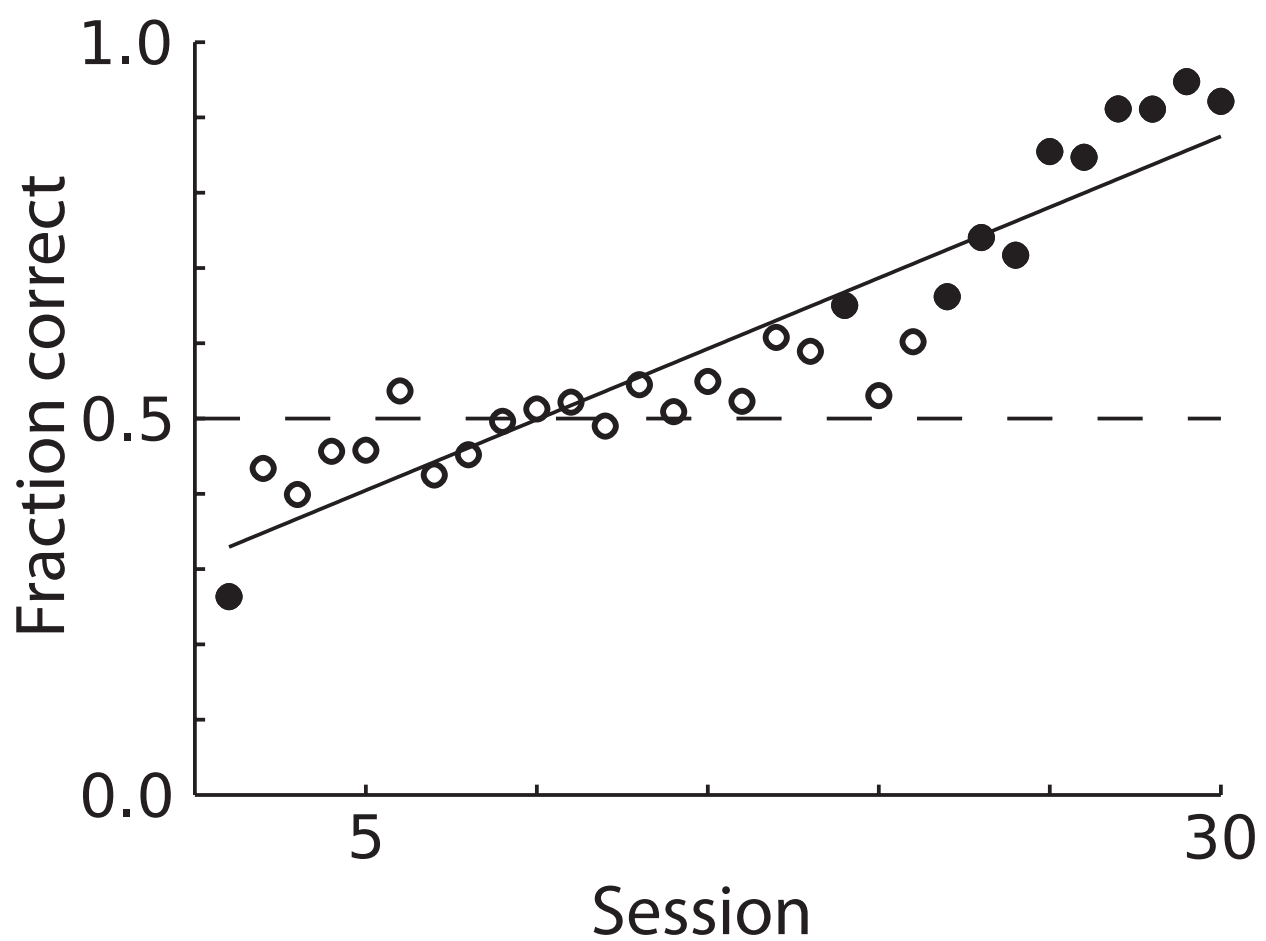

FIGURE 3.11: Learning after a cue-response reversal. Improvement in target discrimination are shown for Monkey G after the association between the ICMS and the response target was reversed. Solid line indicates the line of best linear fit

of $150 \mu \mathrm{s}$ ) and reached 90\% accuracy when ICMS amplitude was at $50 \mu \mathrm{A}$ (Figure 3.12 on the following page). The average proportion of correctly performed trials with an ICMS amplitude greater than $20 \mu \mathrm{A}$ was $88 \%$, which was significantly different from chance $(P<$ 0.001 , Wilcoxon ranked-sum test). For currents less than $20 \mu \mathrm{A}$ the monkey performed at 50\% chance level $(P=0.8)$. Curiously, Monkey $\mathrm{G}$ exhibited better performance when ICMS amplitude exceeded $20 \mu \mathrm{A}$ in the trials immediately following error trials in which the instruction simply repeated. In these trials, the monkey could receive a reward by simply changing the previous erroneous direction, so the instruction, in principle, could be ignored. Nonetheless, for ICMS currents less than $20 \mu \mathrm{A}$ Monkey G performed correctly in only $72 \%$ of trials, which was significantly different from chance $(P<0.001)$, but significantly worse $(P<0.001)$ than the $84 \%$ correct performance with ICMS currents greater than 


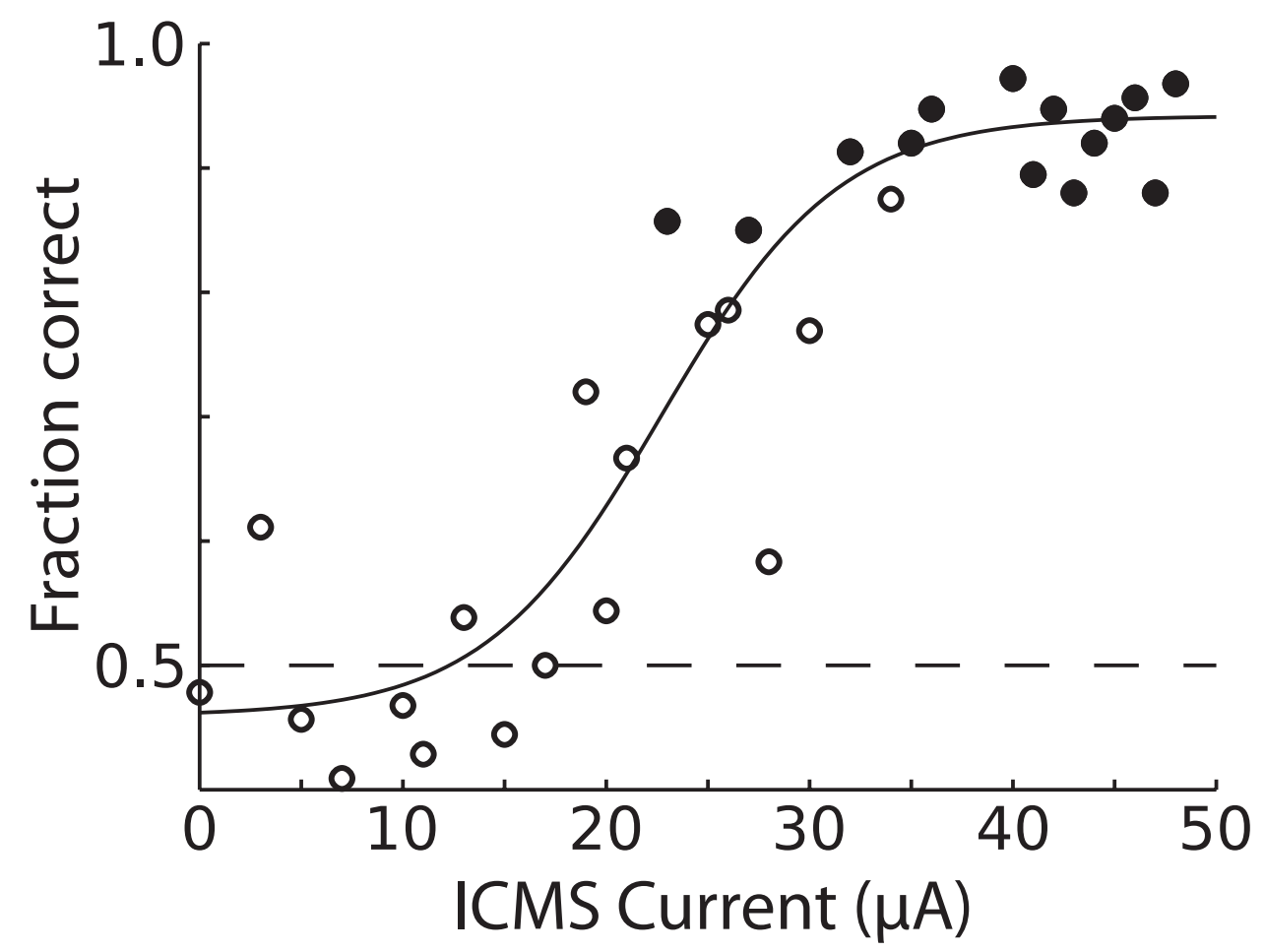

FIGURE 3.12: Psychometric curve for current amplitude. Data was collected with monkey G. On each trial the ICMS current amplitude for the cue pulse train was modulated. Solid curves indicate the line of best sigmoidal fit.

$20 \mu \mathrm{A}$. Thus, ICMS cue clearly benefited the performance even in those trials. This effect was also clear from an analysis of reaction times. The reaction time on regular trials (i.e., rewarded trials preceded by rewarded trials) was $457 \pm 43 \mathrm{~ms}$ (mean \pm standard error) for the currents less than $20 \mu \mathrm{A}$ and $454 \pm 18 \mathrm{~ms}$ for the higher currents. The small $3 \mathrm{~ms}$ average difference was statistically significant $(P<0.001$, Wilcoxon ranked-sum test). In the trials following error trials, the monkey's decisions occurred significantly slower than on regular trials $(P<0.001)$, as indicated by increased reaction times: $785 \pm 60 \mathrm{~ms}$ for currents less than $20 \mu \mathrm{A}$ and $562 \pm 51 \mathrm{~ms}$ for those higher. Thus, higher ICMS currents resulted in a considerable $(223 \mathrm{~ms} ; P<0.001)$ improvement in the reaction time.

Monkey $\mathrm{C}$ learned the vibrotactile cued task on a similar time-course as Monkey G (7 sessions to achieve performance greater than chance and 11 sessions to reach greater than 
90\% accuracy; Figure 3.9 on page 43 ). This animal did not have an implant in $\mathrm{S}_{1}$ and was unable to learn the task instructed by ICMS of the PPC even though training with ICMS continued for 30 sessions.

\subsubsection{BMI with somatosensory input}

In the BMI with somatosensory input, one monkey controlled cursor movements directly by using motor cortical activity while receiving somatosensory instructive signals in the form of $\mathrm{S}_{1}$ ICMS. The second monkey also controlled the cursor using motor cortical activity but, since PPC ICMS was ineffective, received somatosensory signals via vibrotactile stimulation of the hand. The major technical challenge for carrying out these experiments was obtaining simultaneous recordings from large neuronal populations in motor cortical areas concurrent with ICMS to sensory cortical areas. The potential for ICMS pulses occluding spikes was limited to 6 to $12 \%$ of the stimulation interval because electrical artifacts evoked by the pulses were brief (with the primary effect lasting less than $2 \mathrm{~ms}$ ) and easily distinguishable from action potentials. While we recorded neuronal activity during ICMS, we excluded the instruction intervals from the data-set used to train the BMI algorithms. This was done to avoid any influence of the stimulation on the parameters of the extraction algorithm (e.g., occluded spikes interpreted as a decrease in firing rate or neuronal responses to ICMS confused with motor commands). No neuronal activity was excluded during online BMI operations.

Neuronal modulations were observed both during the instruction and during the movement periods of the target choice task. We observed neurons that exhibited increases and decreases in their firing rates during the instruction period, as well as neurons that were directionally selective exclusively during the movement period (Figure 3.13 on the following page). Movement direction could be predicted from neuronal population activity before the monkey moved the joystick (Figure 3.14 on page 49 ). In the early trials, when the monkey performed close to chance level (blue trace), the rise in the predictions occurred before the 


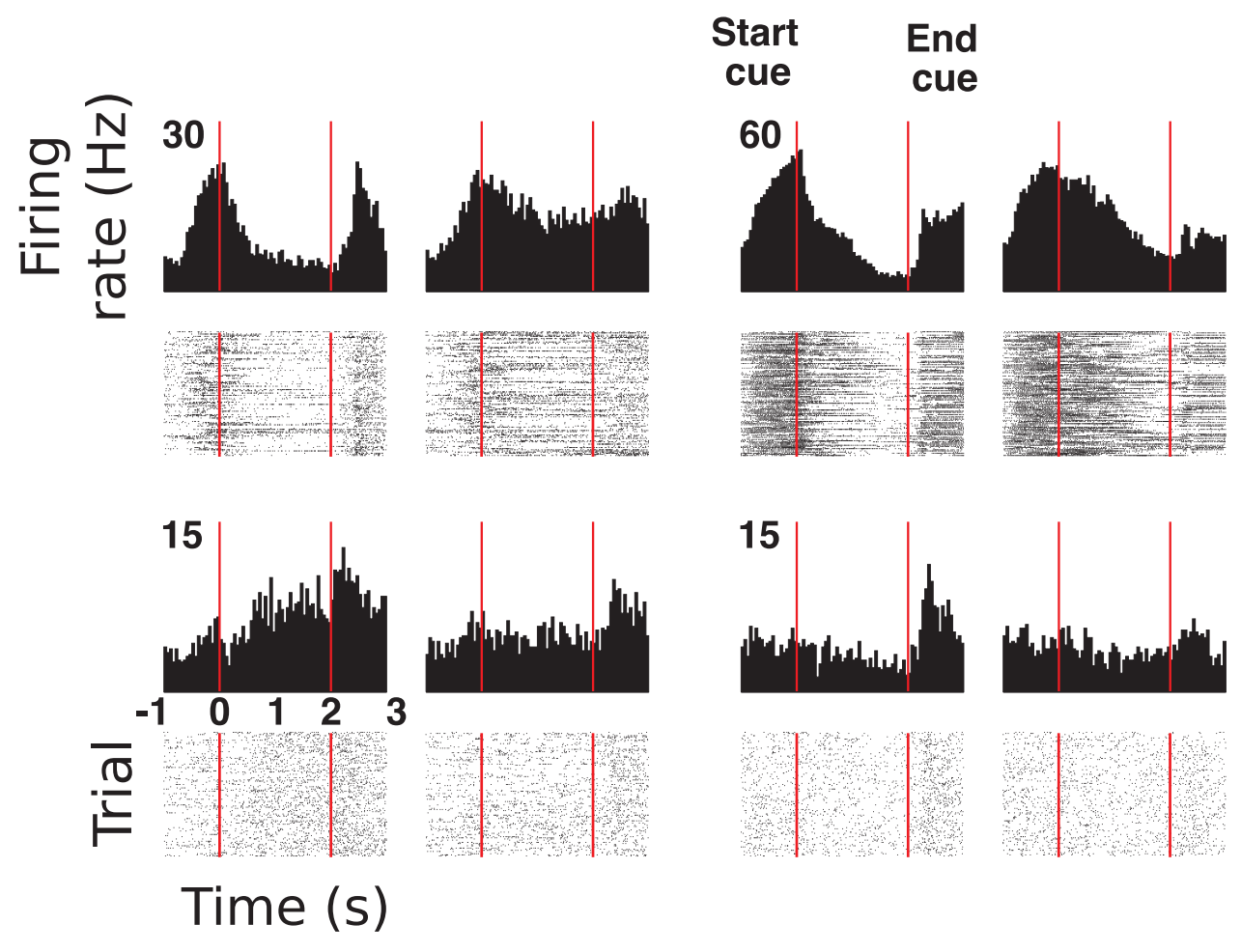

FIGURE 3.13: PETHs of M1 activity during ICMS cueing. Four M1 single units with different modulation patterns recorded from Monkey $\mathrm{G}$ are shown. Trials are aligned on cue onset. The vertical red lines indicate start and end of the cue interval. Left columns indicate ICMS trials during which the monkey moved to the left; right columns indicate movements to the right.

delay. This suggests that the monkey started to form its decision before the stimulus was delivered. In other words, the animal was simply guessing. However, after learning (red trace, Figure 3.14 on the following page), the rise in neuronal prediction occurred subsequent to the instruction period onset and had a steeper slope, indicating a more rapid accumulation of evidence for the reach direction. In control sessions in which no ICMS was presented (black trace, Figure 3.14 on the next page), information about the impending movement was not present in the population activity until $0.5 \mathrm{~s}$ before movement onset. This indicates that in the late sessions, the monkey did not make premature guesses. Instead, the evidence suggests that this monkey formed its target choices based on the pattern of ICMS. Reach direction could also be predicted in brain control (see below; Figure 3.15 on page 50). 


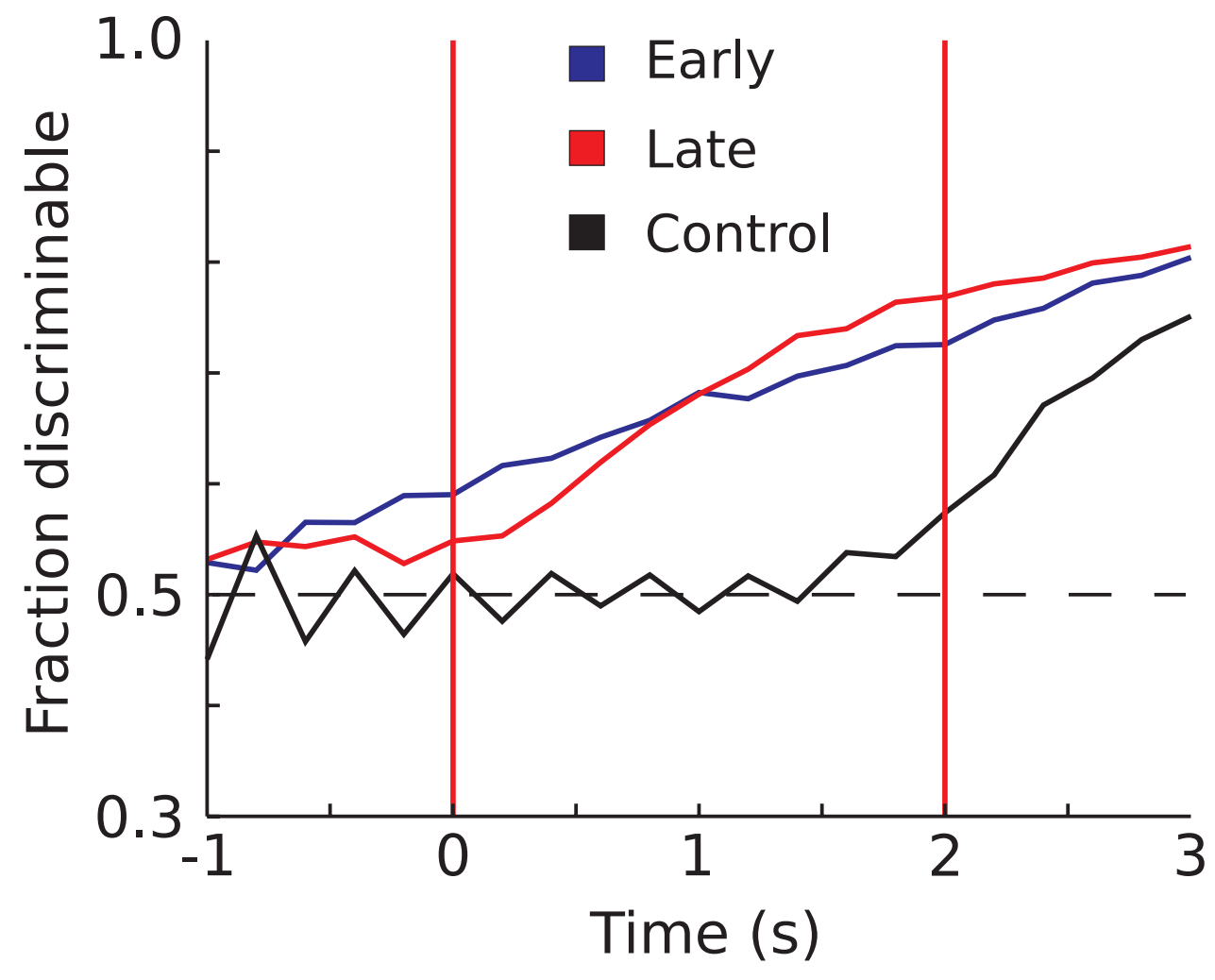

FIGURE 3.14: Average linear discriminant analysis (LDA) predictions of reach direction as a function of time since cue onset show learning-related changes. Blue and red traces indicate averages across early $(n=15)$ and late $(n=15)$ sessions, respectively; control sessions $(n=2)$ are shown in black. Vertical red lines indicate start and end of the cue interval. 


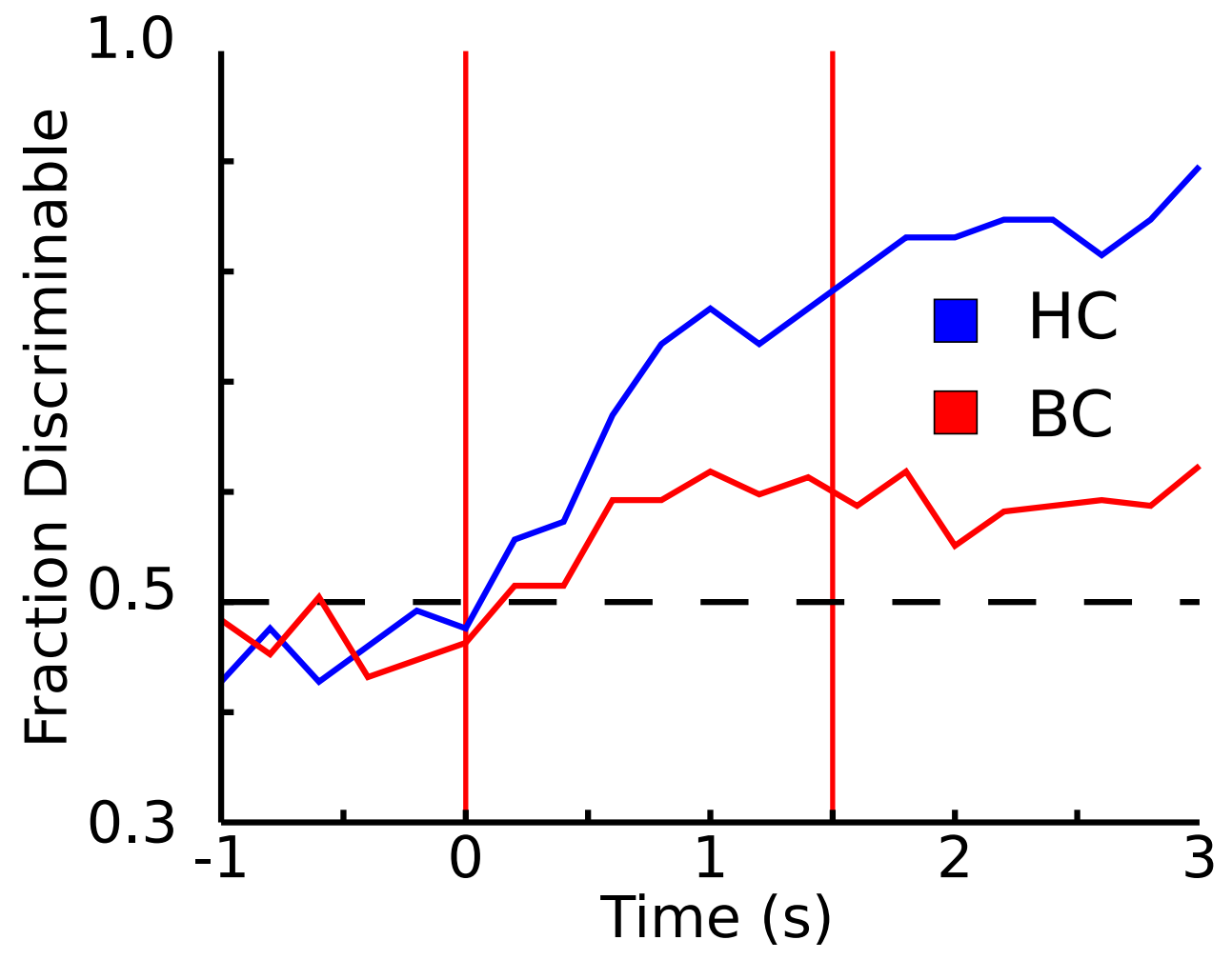

FIGURE 3.15: LDA predictions of reach direction for hand control and brain control. Data is shown from a single session with monkey G. Blue traces indicate hand control (HC); red trace indicates brain control (BC). Vertical red lines indicate start and end of the cue interval. 


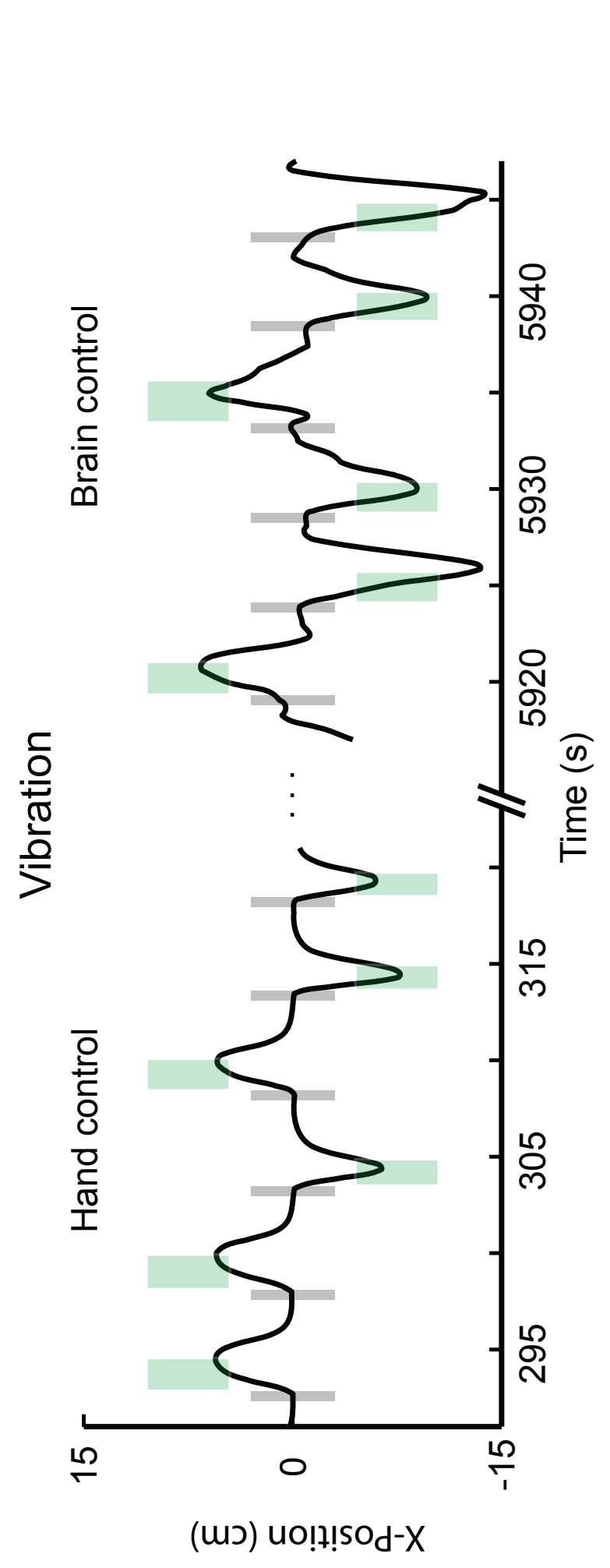

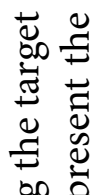

o

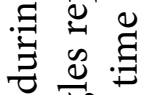

잉

원

के प्ठ

제

क्षे

光

फे के

㝴陆

ㅇํㄹ

들

O 3

凶

당

F 0

ن

उ. पू

灵 䨌

บ $\sigma 0$

퓽 छ

零艺

吾

寻 䓀

30

节

छี

Ð

导苛

$\exists \cup$

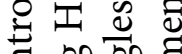

I

을

. 펀

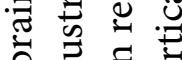

ป气 Е

चै के है

चु

焉

$\ddot{\theta}$ 宩

m

될 可

S.

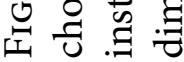




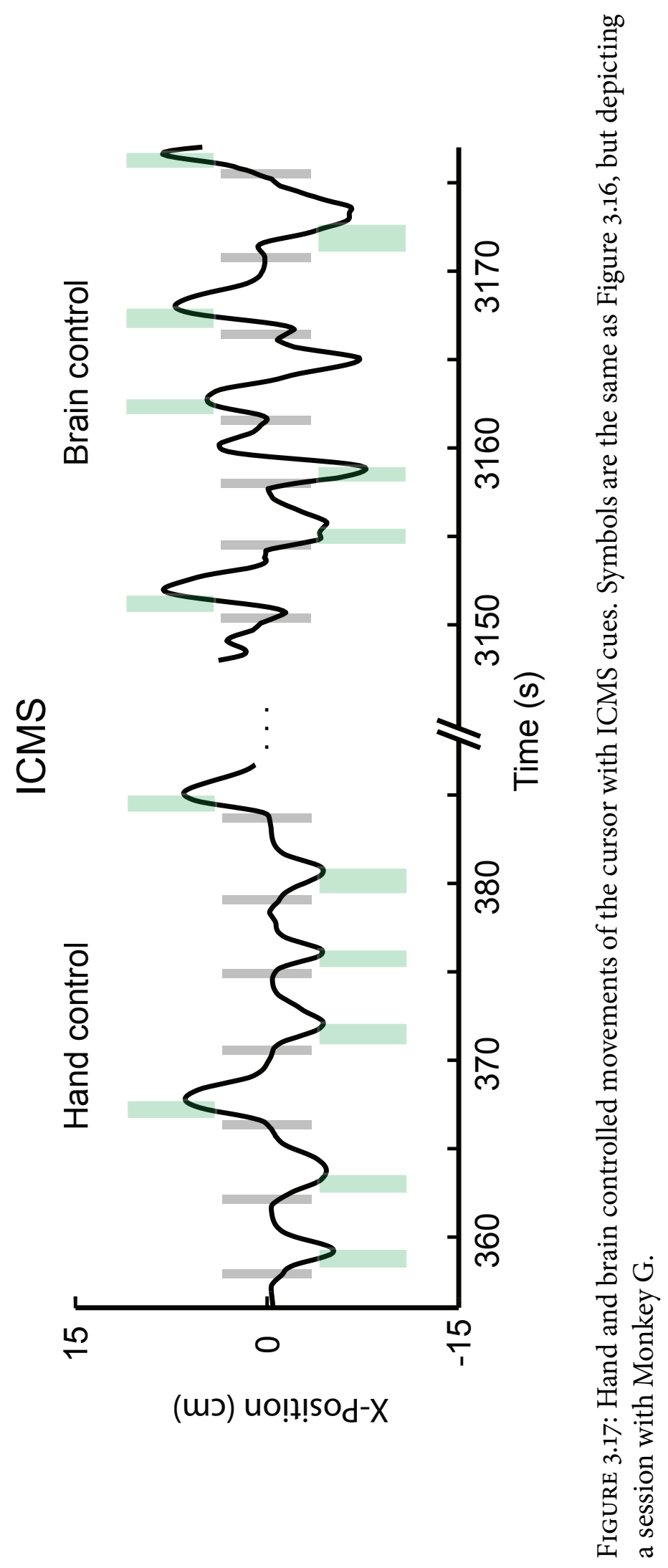




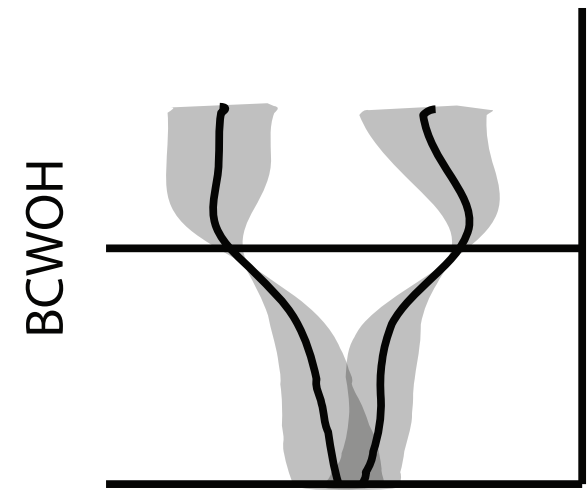

ত্

$\circlearrowleft \approx$

矛总

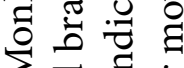

$\bar{Z} \Xi$

声甜新

풍

के

的

๘

方䓪

我

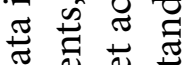

एँ

म

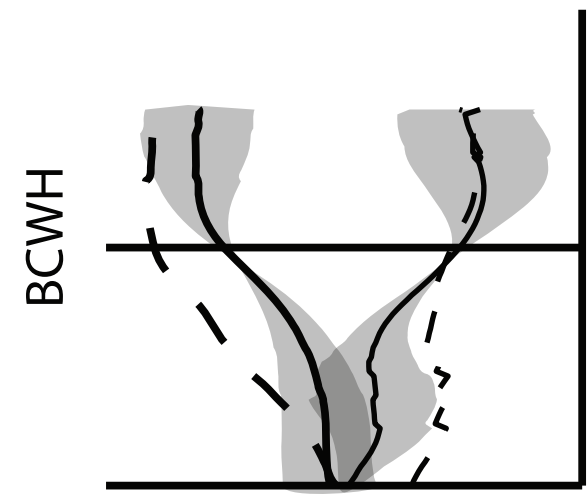

苛

స్త

ธี $\Xi$

오 웜

总声壱

뭉 离

음

ㅇํㅇำ

ส

$\exists \stackrel{\Xi}{\Xi}$

후를

त

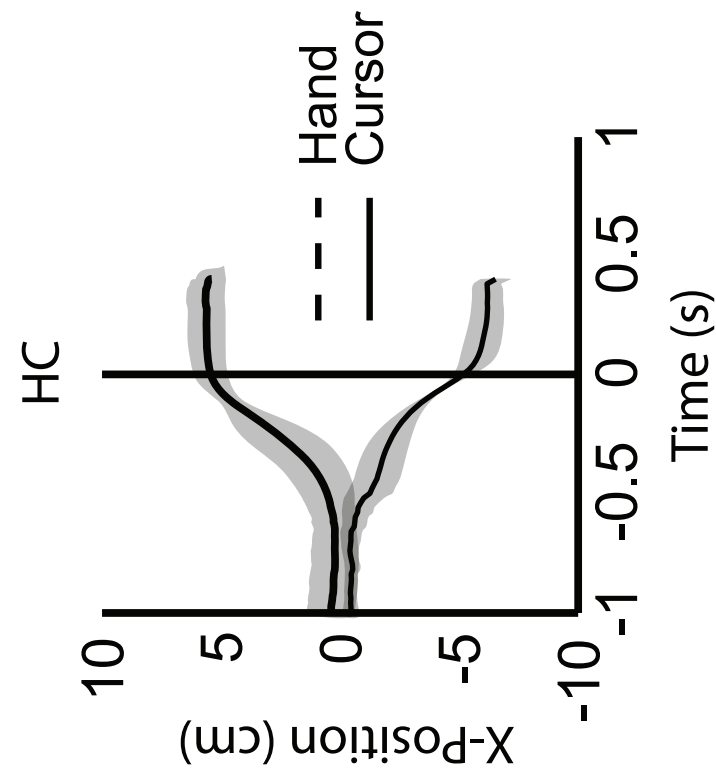

సี

000 约

官苛

$7 \pm$

을

䓀

艺艺芆

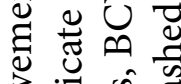

है :

घ $\Xi$ चे

曲 है त

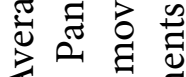

य

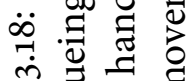

덜

뜽

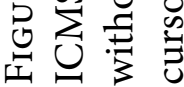


Real-time, direct brain control was instructed by ICMS in sessions with Monkey G (Figure 3.17 on page 52) and by vibrotactile stimulation in sessions with Monkey C (Figure 3.16 on page 51). Monkey $\mathrm{G}$ performed without using the joystick in some sessions (Figure 3.18 on the previous page); Monkey C continued to use the joystick (which was needed to produce vibrotactile stimulation). Both monkeys exceeded 70\% correct performance in brain control. Monkey $\mathrm{G}$ performed above this level in seven sessions of brain control with ICMS. Monkey C started by performing at a 55\% correct performance level in brain control with vibrotactile input, and reached a greater than $80 \%$ performance on the seventh session of training.

\subsection{Discussion}

Altogether, these experiments demonstrate that somatosensory input can be incorporated in BMIs using either vibrotactile stimulation or ICMS of S1. This type of recursive somatosensory input can be considered a form of feedback because the stimulus was contingent on cursor position. The notion that electrical stimulation of cortical areas may reproduce physiological sensations dates back to the pioneering studies of Penfield (Penfield \& Boldrey, 1937). More recently, ICMS of S1 proved to be efficient to reproduce temporal (Romo et al., 1998) and spatiotemporal (Fitzsimmons et al., 2007) parameters of somatosensory inputs. These findings are consistent with our previous demonstration that spatiotemporal patterns of ICMS delivered through chronically implanted multi-electrode arrays are viable over long intervals, leading to steady improvement of the behaviors instructed by ICMS (Fitzsimmons et al., 2007).

\subsubsection{Rate of learning}

As in our previous study (Fitzsimmons et al., 2007), we did not observe an immediate ability of the monkeys to interpret ICMS applied to $\mathrm{S}_{1}$, despite the monkeys being previously overtrained with vibrotactile stimuli. This could indicate a substantial difference between the sensations evoked by skin vibration and ICMS. This result is different from the description 
of Romo and colleagues (Romo et al., 1998) who reported almost immediate learning with ICMS of S1. One explanation for this discrepancy is the specific S1 subdivision implanted. Romo and colleagues (Romo et al., 1998) implanted area $3 \mathrm{~b}$, which is upstream to area 1 implanted in our study. Additionally, their experiment included a number of initial test sessions during which learning to discriminate ICMS could have occurred.

When Monkey G finally did start learning the ICMS cue, its learning took a very rapid time course (Figure 3.10 on page 44 ). One possible interpretation is that the sensations evoked by ICMS of area 1 were quite unlike skin vibration. Another explanation is that no sensations were initially evoked and that repeated exposure to ICMS was required for any sensation to develop. Further experiments will be required to better understand factors involved in the development of sensation due to S1 ICMS. Although ICMS of area 1 may not initially evoke sensations very similar to skin vibration, as follows from our present study and previous work (Fitzsimmons et al., 2007), this does not at all preclude its use in BMI applications.

\subsubsection{Mechanism of action of ICMS}

The action of $\mathrm{S}_{1}$ ICMS was not as trivial as stimulating M1 through the direct projections from S1 to M1. M1 recordings did not reveal any ICMS-evoked responses per se in this cortical area. The presence of such evoked responses would be consistent with the animal's behavioral response being mediated by a simple detection-like signal, mediated by ICMS, and activating M1 directly. Instead, we observed the occurrence of a gradual build up of preparatory neuronal activity that did not merely mirror the constant frequency of ICMS (Figure 3.13 on page $48,3.14$ on page 49 ). Moreover, there was a $33 \%$ reaction time difference observed between low ICMS current amplitudes (less than $20 \mu \mathrm{A}$ ) and high ICMS current amplitudes (greater than $20 \mu \mathrm{A}$ ) for correction trials in which the monkey knew the correct response. This difference provides further evidence that the monkey was attending to the ICMS cue. Finally, the monkey successfully learned an ICMS-reach association reversal 
from a target contralateral to the stimulated hemisphere to a target ipsilateral to the stimulus (Figure 3.11 on page 45 ). This would not happen if the monkey simply reached toward the hemifield contralateral to M1 which was excited by the projections from S1.

\subsubsection{Ineffectiveness of PPC stimulation}

The second monkey was unable to learn to discriminate ICMS of PPC, even after 30 daily sessions. This result mirrored our previous observation in an owl monkey that was unable to learn to discriminate PPC stimulation but later learned to discriminate ICMS delivered to S1 (Fitzsimmons et al., 2007). Therefore, it is conceivable that PPC cannot be used for this type of sensory instruction, or that use of PPC may require much longer training or require different parameters. This result suggests that for ICMS to instruct target selection, it may not be sufficient to simply inject current to any part of the cortical network that controls reaching movements, such as PPC. Stimulation of primary sensory areas of the cortex (and possibly thalamus (Heming et al., 2010)) appears to be most effective for sensory substitution, as follows from our present study, as well as from previous studies conducted by us (Fitzsimmons et al., 2007) and others (Richer et al., 1993).

\subsubsection{Sensory Substitution}

Several recent studies described the addition of vibrotactile sensory channels to EEG-based BMIs (Chatterjee et al., 2007; Cincotti et al., 2007). While sensory substitution using haptic devices (Vidal-Verdú \& Hafez, 2007) is clearly an important development that will benefit patients, sensorizing BMIs with ICMS is potentially capable of surpassing sensory substitu-

tion. Indeed, by using ICMS to directly target the cortical areas deprived of sensory input after neurological injury, disease, or limb loss, we endeavor to directly use intact brain functionality rather than delegating sensory processing to other structures.

The present demonstration of a BMI instructed by ICMS has brought together two experimental paradigms that were previously studied in isolation: real-time BMI control 
based on neuronal ensemble recordings from cortical motor areas (Wessberg et al., 2000; Serruya et al., 2002; Taylor et al., 2002; Carmena et al., 2003; Moritz et al., 2008; Velliste et al., 2008) and ICMS of S1 as a means to evoke somatic sensations (Romo et al., 1998; Talwar et al., 2002; Butovas \& Schwarz, 2007; Fitzsimmons et al., 2007; London et al., 2008). Our results show that, with training, monkeys can learn to select their reaching movements using somatosensory cues, and one monkey reported here learned to utilize S1 ICMS to mediate BMI-controlled reaching movements.

We have established the feasibility of moving from a brain-machine interface to a brainmachine-brain interface in which artificial actuators and brain tissue are directly connected in a bidirectional way. These results lead us to suggest that in the future, ICMS delivered through chronically implanted multi-electrode arrays may be incorporated into many BMI paradigms and used for delivering artificial sensory feedback directly to the subject's brain. 


\section{4 \\ Sensory feedback}

\subsection{Abstract}

$\left\{\begin{array}{l}\text { RAIN-MACHINE INTERFACES (BMIs) use neuronal activity to control external actuators. } \\ \text { While BMIs aim to enact sensorimotor functions, so far they have lacked somatic }\end{array}\right.$ sensation. We developed a brain-machine-brain interface (BMBI) that enabled artificial tactile sensation through intracortical microstimulation (ICMS) of primary somatosensory cortex (S1). Monkeys controlled a virtual-reality upper limb avatar with a BMBI that derived motor commands from the extracellular neuronal activity of the primary motor cortex (M1). ICMS feedback occurred when the avatar touched virtual objects. Monkeys operated this BMBI to search through sets of visually identical objects and select those with particular textures without relying on somatic sensation from their real bodies. This suggests that motor neuroprostheses can implement ICMS feedback to generate somatic perceptions from mechanical, robotic, or even virtual prostheses. 


\subsection{Introduction}

Brain-machine interfaces (BMIs) have undergone impressive development during the last decade, driven by the expectation that neuronal activity recorded from the brains of persons with paralysis may be used to control the movements of prosthetic limbs or exoskeletons (Lebedev \& Nicolelis, 2006; Nicolelis \& Lebedev, 2009). Starting with the simple control of a one degree-of-freedom artificial actuator (Chapin et al., 1999), BMIs have evolved to perform reaching (Wessberg et al., 2000; Taylor et al., 2002) and grasping (Carmena et al., 2003) actuated by robotic arms (Velliste et al., 2008) and muscle stimulators (Moritz et al., 2008). Nonetheless, BMIs have yet to achieve the degree of accuracy provided by intact limbs. This is due, in part, to feedback about BMI actuators being almost exclusively limited to vision, and lacking the rich somatosensory signals needed for the execution of dexterous movements (James et al., 2007; Ribot-Ciscar et al., 2003).

The importance of such somatic feedback can be inferred from the impairments of grasping and manipulating objects that result from deficits in the sensation of slip (Johansson \& Westling, 1984) or load force (Flanagan \& Wing, 1993). Tactile feedback is essential for dexterous tool use (Katz \& Krueger, 1989), enabling, for instance, a surgeon to wield a scalpel, or a dentist a drill, with great precision. The users of upper limb prostheses place great importance on the ability to perform functions with minimal visual attention (Atkins et al., 1996), and it is reasonable to expect that they would benefit from tactile feedback.

We have envisioned the development of a sensorized neuroprosthesis that simultaneously extracts commands from motor areas of the brain to control an actuator, while delivering concurrent feedback through an artificial sensory channel directly to the somatosensory areas of the brain (Lebedev \& Nicolelis, 2006; Nicolelis \& Lebedev, 2009; O'Doherty et al., 2009). The idea of artificial sensation for prostheses has been previously explored in the context of sensory substitution (Kaczmarek et al., 1991), either by means of vibrotactile (Cincotti et al., 2007) or electrotactile (Scott et al., 1980) stimulation or targeted nerve reinnerva- 


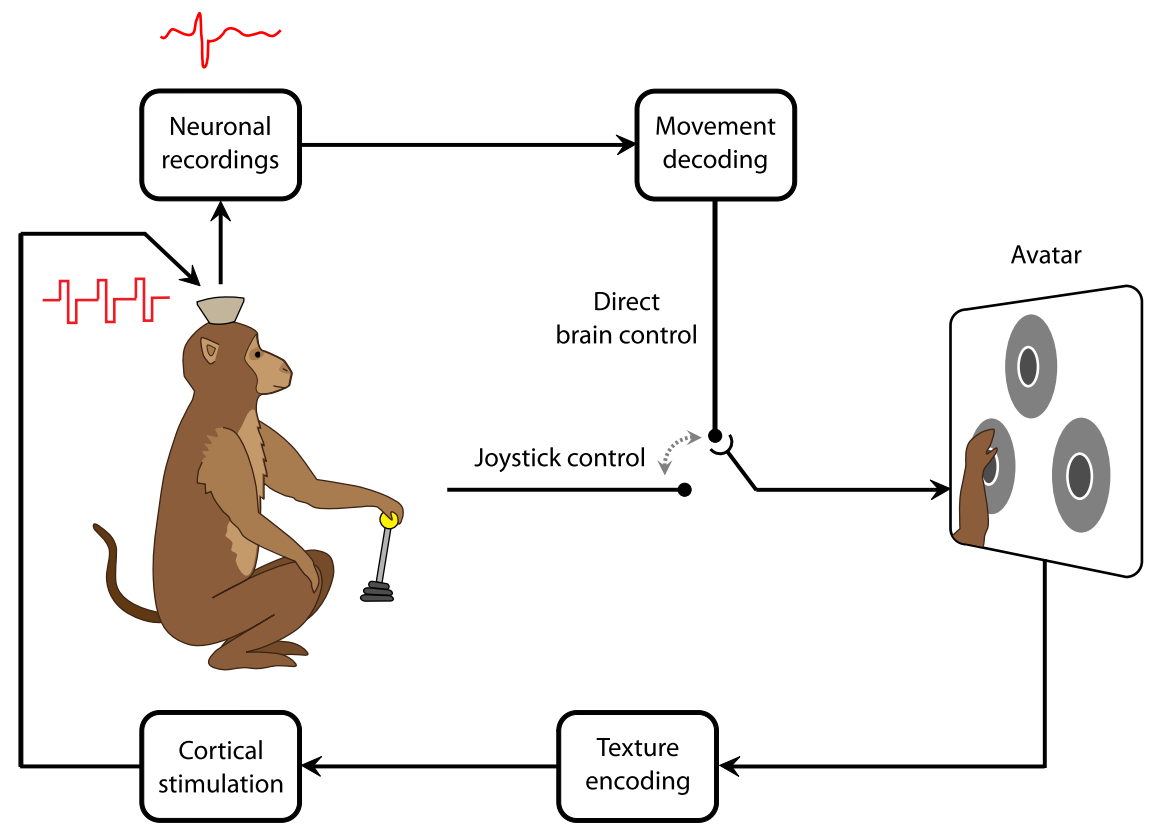

FIGURE 4.1: The brain-machine-brain paradigm. A monkey is seated in front of a display on which an avatar and virtual objects are projected. Movement intentions are decoded from populations of M1 neurons and used to drive a BMI. Artificial tactile feedback about virtual objects is delivered to populations of S1 neurons via patterns of ICMS.

tion (Marasco et al., 2009). However, peripheral sensory substitution faces several pitfalls: limited channel capacity due to substitute mechanoreceptor density; limited mobility due to the size of the stimulation device; and very limited application for spinal cord injured and deafferentated patients, who are unable to sense most of their own bodies.

The brain-machine-brain interface (BMBI) design developed in the present study (Figure 4.1) employed a different strategy by delivering tactile feedback via direct activation of the primary somatosensory cortex $\left(\mathrm{S}_{1}\right)$ of monkeys, using multi-channel intracortical microstimulation (ICMS). This approach extended previous demonstrations that cortical stimulation evokes sensations in humans (Richer et al., 1993) and that temporal (Romo et al., 1998) and spatiotemporal (Fitzsimmons et al., 2007) ICMS parameters can be discriminated by non-human primates. The rhesus monkeys learned to use the activity of cortical motor 
neurons to control the movements of an upper limb avatar, while simultaneously processing a stream of artificial tactile feedback. Under this BMBI control, monkeys learned to reach to virtual objects, discriminate them by their texture, and select those with particular tactile properties-all without apprehending the objects with their own hands or engaging skin mechanoreceptors.

\subsection{Materials and methods}

\subsubsection{Electrode arrays}

Each monkey was implanted with four 96-channel revision A microwire arrays. See Section 2.2 for the details of array topology. Each hemisphere received two arrays: one in the upper and one in the lower limb representation areas such that a single array corresponded to the sensorimotor cortical regions associated with a single limb. Each array sampled neurons in both primary motor $\left(\mathrm{M}_{1}\right)$ and primary sensory $\left(\mathrm{S}_{1}\right)$ cortex. In this study, we used recordings from the right hemisphere arm arrays in each monkey, since both manipulated the joystick with their left hands.

\subsubsection{Tasks}

The monkeys were trained to manipulate a computer cursor or a virtual reality arm (avatar) and to make reaches towards objects displayed on a computer monitor. The objects were visually identical, but exhibited different tactile properties as conveyed by an artificial somatosensory input. The objective of the behavioral task was to find and select the object with the appropriate artificial tactile properties. If the correct object was selected, the monkeys were rewarded with a drop of fruit juice.

In manual control, monkeys moved the cursor or the avatar arm with a joystick. Each trial commenced when the monkey held the joystick with their working hand. Then, a target appeared in the center of the screen. The monkey had to hold the actuator (cursor or avatar

arm) within that center target for a hold time, $t_{\text {hold }}$, uniformly drawn from a half-closed 
interval:

$$
t_{\text {hold }} \in(0,2000] \mathrm{ms}
$$

After this, the central target disappeared and was replaced by a set of virtual objects radially arranged about the center of the screen. Each of these consisted of a central response zone and a peripheral feedback zone. These regions were visually distinguished by their shading. Tactile feedback was delivered whenever the actuator was placed within an object feedback zone or corresponding response zone. The monkey was allowed to explore the virtual objects in any sequence. A trial was concluded when the monkey placed the actuator within one of the object response zones for a hold interval (80o to 1300 milliseconds, fixed interval, depending on the session), a trial timeout was reached (typically $10 \mathrm{~s}$ ), or the monkey released the joystick handle (in manual control trials). The next trial commenced after an inter-trial interval (500 ms).

The sequence of events was the same during brain control trials, when cortical ensemble activity rather than joystick movements controlled the cursor on the screen. In the majority of brain control sessions monkeys continued to move the joystick although it was disconnected from the actuator. In some sessions, the joystick was removed from the behavioral setup. For these, each new trial commenced immediately following the previous inter-trial interval.

The monkeys were exposed to behavioral tasks of increasing difficulty. For tasks I through III, monkeys chose from a set of two objects. For Task I, the monkeys had to choose between RAT and NAT for fixed object locations. For Task II, RAT and NAT were presented on the screen at different angular locations on each trial. For task III, object number and spatial arrangement were the same as in Task II, but RAT and UAT were used. For Task IV, three objects were used (RAT, UAT and NAT), whose arrangement on the screen varied from trial to trial. Finally, for Task V, the monkey avatar arm replaced the computer cursor. 


\subsubsection{Catch trials}

In some sessions, a small percentage of trials (typically $1 \%$ ) were designated as catch trials. For these trials, the microstimulator delivered pulse trains with zero amplitude, however all other experimental procedures remained unchanged. This control allowed us to confirm that there were no unintentional sources of information that the monkeys could exploit to perform the tasks.

\subsubsection{Algorithms}

Cortical activity was transformed into actuator movements using an Nth-order Unscented Kalman filter (UKF; Li et al., 2009) that we have previously shown to be superior to linear approaches for decoding arm movements. We used up to a 1oth-order UKF in some sessions, but in most we found that the 3 rd-order UKF was sufficient. The filter parameters were fit based on the hand movements of the monkeys while they performed the task using a joystick, or in the final experiments, based on passive observation of avatar movement while the monkeys' arms were restrained.

\subsubsection{ICMS}

Symmetric, biphasic, charge-balanced pulse trains of ICMS were delivered in a bipolar fashion through adjacent pairs of microwires. The pairs of microwires used for stimulation had clear sensory receptive fields in the upper limb (monkey $M$ ) or lower limb (monkey $\mathrm{N})$. For monkey $\mathrm{M}$, the anodic and cathodic phases of stimulation had a pulse width of $105 \mu$ s; for monkey $\mathrm{N}$ the pulse width was $200 \mu$ s. The anodic and cathodic phases of the stimulation waveforms were separated by $25 \mu \mathrm{s}$.

\subsubsection{Interleaved ICMS and recordings}

To achieve concurrent afferent and efferent operations we implemented an interleaved scheme of alternating recording and stimulation intervals. As noted above, our BMI had a 
$10 \mathrm{~Hz}$ update rate. That is, $100 \mathrm{~ms}$ of past neural data are used to make predictions about the desired state of the actuator. We broke up each 100-millisecond interval into two $50 \mathrm{~ms}$ sub-intervals. In the first sub-interval (Rec), neural activity was recorded as usual and the spike count measured was used to estimate the firing rate for the whole 100-milisecond interval. The second sub-interval (Stim) was reserved exclusively for delivering ICMS; all spiking activity occurring in this sub-interval was discarded.

Whenever the actuator was in contact with a virtual object at the start of a Stim interval, an ICMS pulse train was delivered. For RAT, nine pulses of ICMS were delivered; For UAT, 18 pulses of ICMS were delivered; for NAT, no pulses of ICMS were delivered. The neural activity in the Stim interval was ignored even in the case of NAT, so that there would be no bias induced by ICMS-occluded neural data. In this way, the BMI operated at $10 \mathrm{~Hz}$ while allowing sensory feedback to occur at the same rate.

\subsubsection{Perievent time histograms (PETHs)}

PETHs were calculated using conventional methods, and included centering records of neuronal discharges on particular task events (e.g. object onset) and representing neuronal rates as binned counts of discharges. The data was binned into $50 \mathrm{~ms}$ wide bins. Periods during which ICMS trains were delivered were excluded from these analyses because ICMS artifacts masked neuronal discharges. In these (blanked) bins, the firing rate was assumed to be unchanged from the previous $50 \mathrm{~ms}$ window. These bins were then combined into $100 \mathrm{~ms}$ wide bins for visualization, mirroring the technique used for online predictions.

\subsubsection{Directional tuning curves}

Directional tuning curves were calculated from neuronal rates within a $250 \mathrm{~ms}$ interval following the time instance when the actuator entered the borders of an object. A vector

pointing from the screen center to the center of the object provided the corresponding direction. If the object was associated with ICMS, firing rates were corrected by excluding 

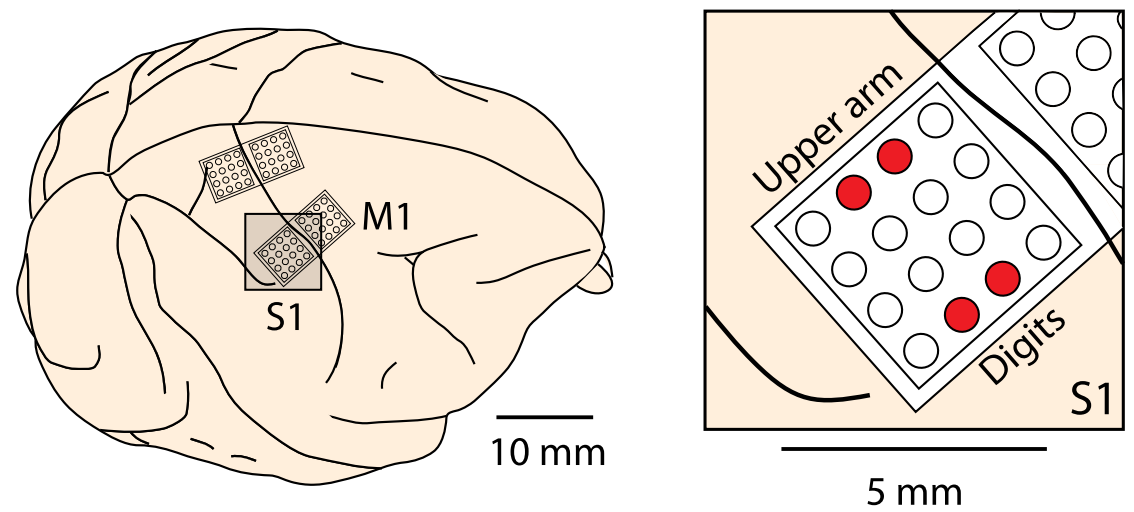

FIGURE 4.2: Implants for ICMS feedback experiment. A. The monkeys were implanted with microwire arrays targeting $\mathrm{M}_{1}$ and $\mathrm{S}_{1}$ of the upper and lower limbs. B. Receptive fields in $\mathrm{S}_{1}$ exhibited typical somatotopic organization. Channels used for stimulation with monkey $\mathrm{M}$ are accented in red.

the $50 \mathrm{~ms}$ periods where ICMS was delivered and it was assumed that the firing rate stayed constant during the excluded intervals.

\subsection{Results}

\subsubsection{Active touch}

Two monkeys ( $\mathrm{M}$ and $\mathrm{N}$ ) were chronically implanted with multielectrode arrays in several subregions of the primary motor (M1) and $\mathrm{S}_{1}$ cortices (panel A of Figure 4.2). For the purposes of this study, we simultaneously recorded the activity of up to 250 Mi neurons contralateral to the working arm. The monkeys learned to perform the reaching task using a hand-held joystick (hand control, HC) that moved a cursor (later, an avatar arm) to select virtual objects displayed on a computer screen (Figure 4.1 on page 60). The task required searching through a set of up to three objects, selecting one with a particular artificial texture (AT), as represented by patterns of intracortical microstimulation and then indicating the 


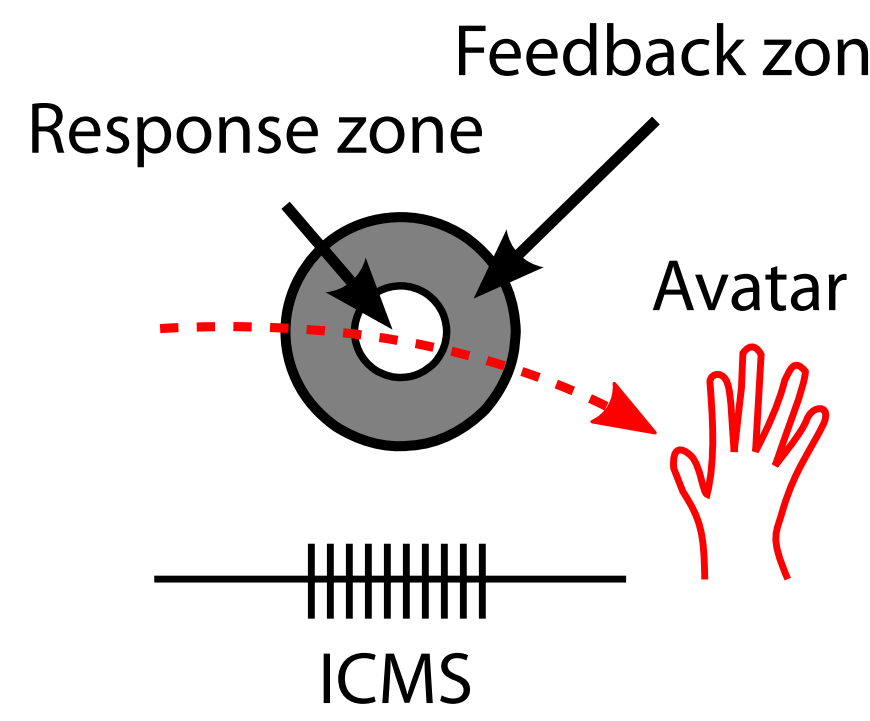

FIGURE 4.3: ICMS feedback stimulation paradigm. Objects on the screen consisted of a central response zone surrounded by a feedback zone. Movement of the avatar though the feedback or response zone triggered the delivery of ICMS pulse trains representing artificial textures.

selection by holding the actuator over that object for reward (Figure 4.3). The task varied in the number of objects on the screen, AT-object association, and the actuator type (cursor or avatar; see all 5 task versions in panel A of Figure 4.7 on page 69 ). Subsequent to training with HC, the joystick was disconnected and the monkeys moved the virtual actuator with their brain activity alone (brain control, BC) using previously developed algorithms (Carmena et al., 2003; Lebedev et al., 2005; Li et al., 2009). This defined the motor loop of our BMBI. Further details about brain control are found in Chapter 2.

The sensory loop of the BMBI mimicked active touch (Lederman \& Klatzky, 1987): when the actuator was placed over an object, ICMS of S1 provided feedback about the object's AT. With monkey M, the upper-limb hand representation area of $S_{1}$ was used as the target for ICMS (panel B of Figure 4.2 on the previous page), so the monkey experienced putative sensations in its hand. With monkey N, ICMS was instead applied to the lower-limb 

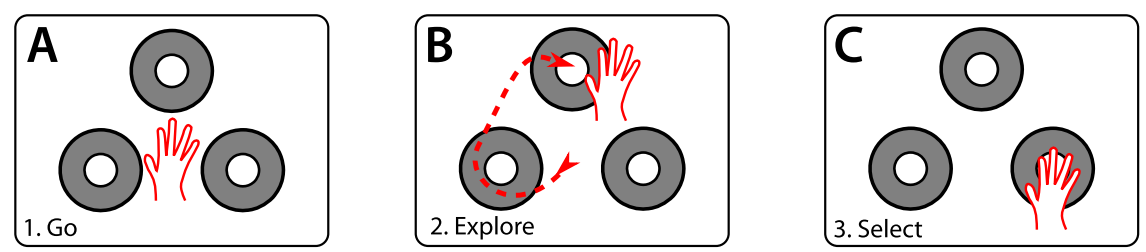

FIgURE 4.4: ICMS feedback task structure. A. Monkeys initiated a trial by holding the avatar in the center of the screen until several peripheral objects appeared (500 to $1300 \mathrm{~ms}$, random per trial). B. Next, the monkeys freely explored the objects. C. Finally, an object was selected by holding the avatar within the response zone for 500 to $1300 \mathrm{~ms}$ (depending on the monkey and session).

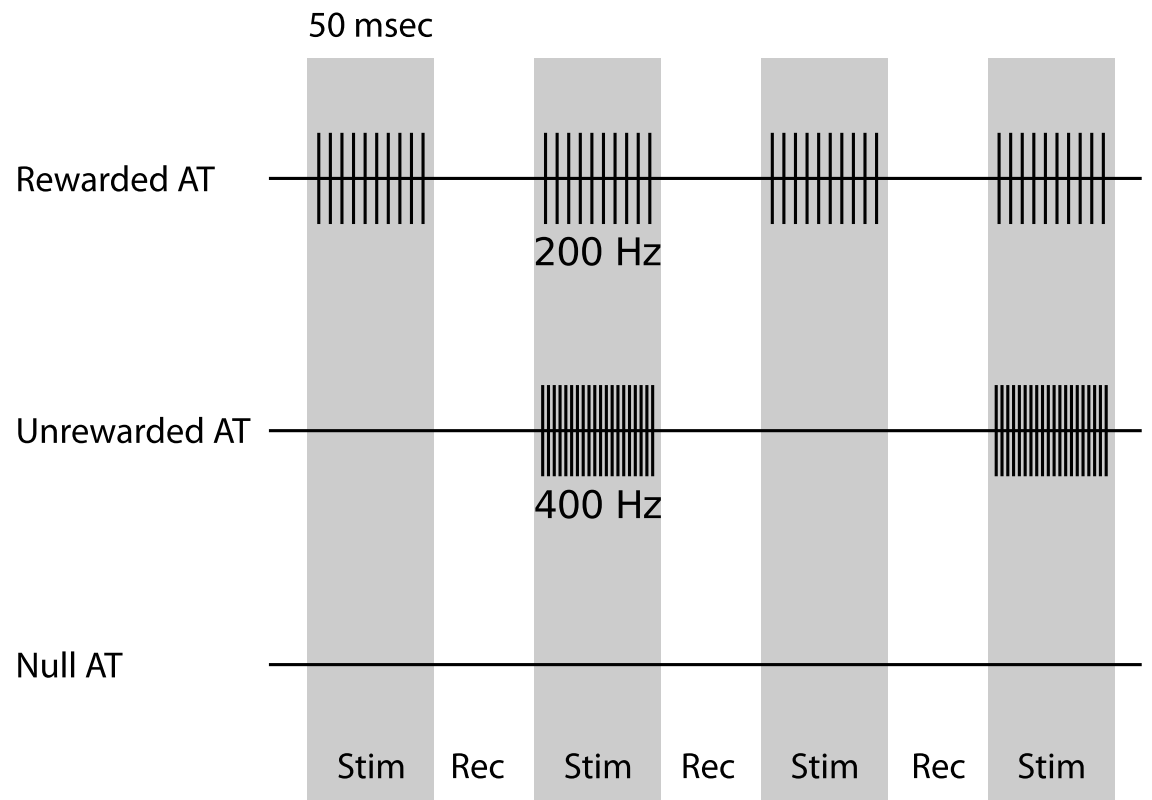

FIGURE 4.5: ICMS patterns delivered for the artificial textures. Schematic of the ICMS patterns delivered for the rewarded, unrewarded, and null ATs. Each vertical line indicates biphasic stimulation waveform. The RAT consisted of $200 \mathrm{~Hz}$ ICMS delivered in $10 \mathrm{~Hz}$ packets; the UAT consisted of $400 \mathrm{~Hz}$ ICMS delivered in $5 \mathrm{~Hz}$ packets. Stimulation (Stim) and recording $(\mathrm{Rec})$ intervals were $50 \mathrm{~ms}$ in duration and alternated at $20 \mathrm{~Hz}$ (50\% dutycycle). 


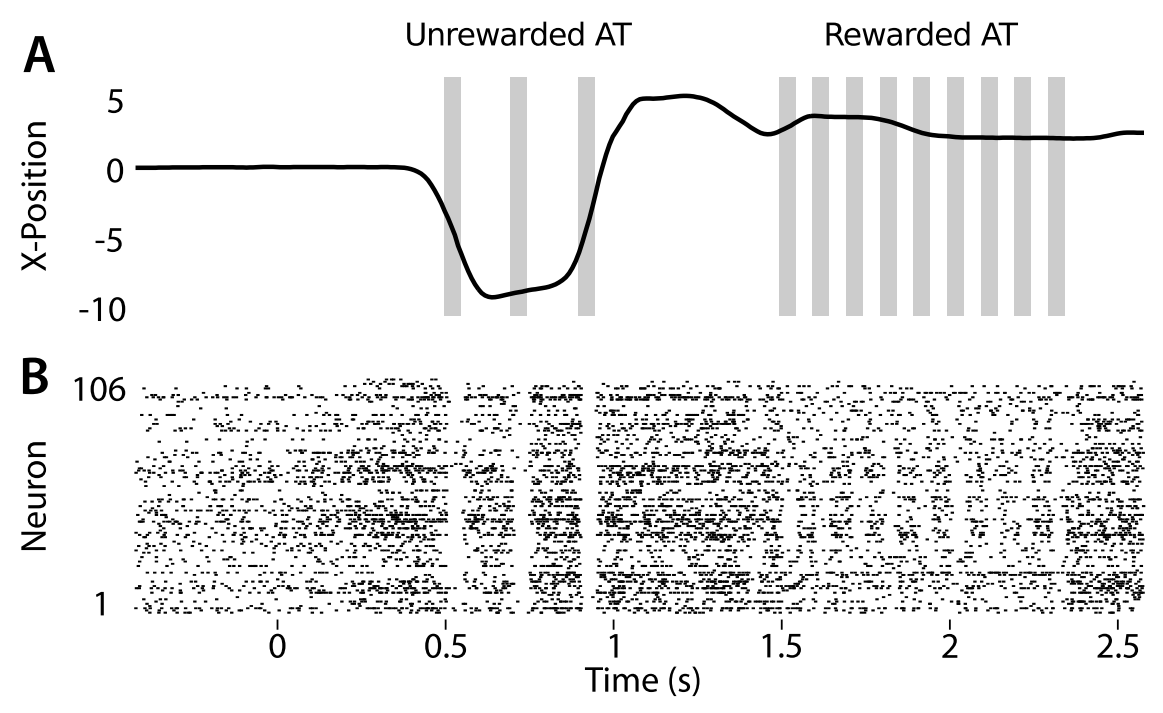

FIGURE 4.6: Example of active reach with concurrent ICMS feedback. A. The x-component of avatar movements are shown for a single trial as the avatar passes through the UAT object and selects the RAT object. Gray bars indicate Stim intervals as in Figure 4.5. A. Rastergram of M1 neurons simultaneously recorded during the movement in panel A.

representation area of S1. As ICMS artifacts masked neuronal activity for 5 to $10 \mathrm{~ms}$ after each pulse (Figure 4.6), we alternated recording and stimulation intervals at a $20 \mathrm{~Hz}$ clock rate (Figure 4.5 on the previous page) to avoid interference with recordings.

Virtual textures were represented by ICMS patterns consisting of high-frequency trains of ICMS pulses presented in packets at a lower secondary frequency (see Figure 4.5 on the preceding page). Up to three visually identical objects were rendered, each with a unique AT. The AT associated with reward (rewarded artificial texture, RAT) consisted of $200 \mathrm{~Hz}$ ICMS delivered in $10 \mathrm{~Hz}$ packets. The unrewarded comparison ATs were represented either by $400 \mathrm{~Hz}$ ICMS delivered in $5 \mathrm{~Hz}$ packets (unrewarded artificial texture, UAT) or by an absence of ICMS (null artificial texture, NAT).

BMBI performance improved with training. In the simplest task, Task I, monkeys discriminated between two objects, one with RAT and one with NAT (see panel A of 

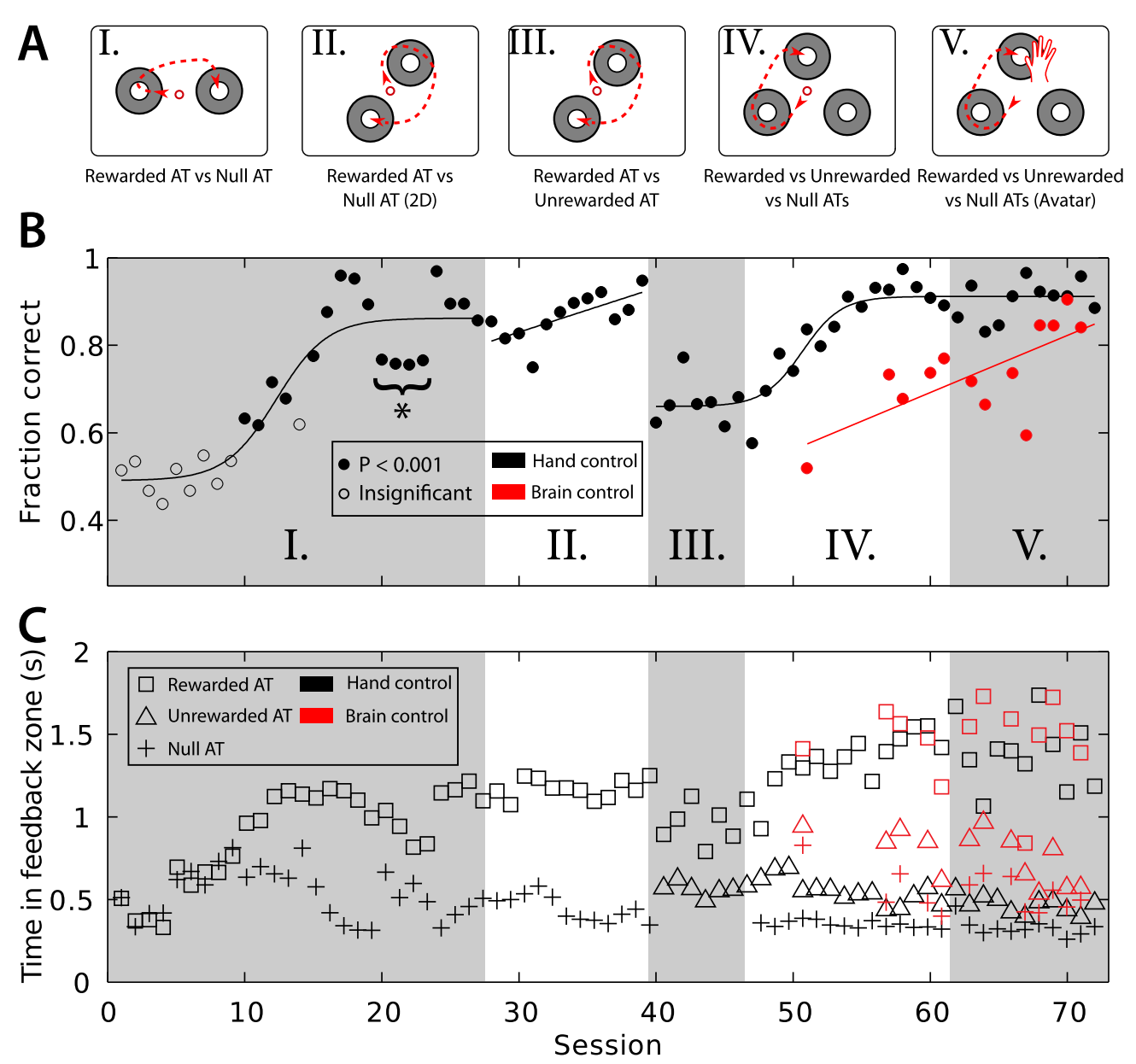

FIGURE 4.7: Learning to use ICMS feedback. A. Diagram of behavioral task variations. Each sub-panel corresponds to a shaded region in panels B and C. B. Summary of the behavioral performance of monkey $\mathrm{M}$ for 72 sessions. Each circle shows the mean performance. Filled circles depict sessions with performance significantly different from chance. Black circles indicate HC; red circles indicate BC. Curves are the linear and sigmoidal lines of best fit. Shaded regions labeled with Roman numerals correspond to the task variations depicted in panel A. Sessions indicated by the asterisk were used to compute the psychometric curve in Figure 4.8. For these, only currents above perceptual threshold were used to compute the mean performance shown here. $\mathbf{C}$. The mean time spent in each of the three possible feedback zones is shown for the same sessions as in panel B. Squares, triangles, and crosses correspond to RAT, UAT, and NAT, respectively. Black symbols indicate HC sessions; red symbols indicate BC sessions. 


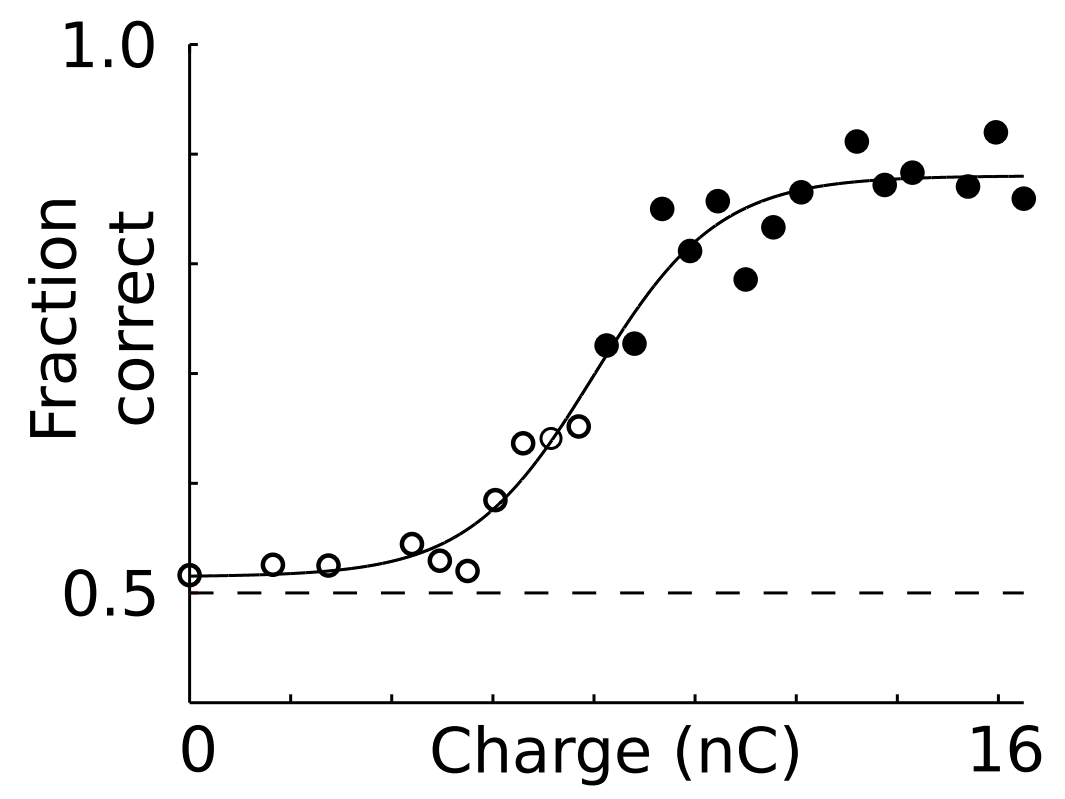

FIGURE 4.8: Psychometric curve of ICMS charge per waveform phase needed for monkey M to successfully perform task I (see asterisk in panel B of Figure 4.7 on the preceding page). Symbols as in Figure 4.7; $\mathrm{n}=4292$ trials.

Figure 4.7 on the previous page). To ensure that the monkeys learned the task objectives even before ICMS introduction, both were first trained in a variant of Task I in which RAT was represented by a vibration of the joystick handle (see Figure 4.9 on the following page). Despite this prior training, it took the monkeys several experimental sessions to learn the ICMS task (see panel B of Figure 4.7 on the previous page). Monkey M achieved performance significantly different from chance after nine sessions; monkey $\mathrm{N}$ after $4(P<0.01$, one-sided binomial test), demonstrating that ICMS delivered to the leg representation area of S1 was effective for AT feedback. Psychometric analysis of stimulation amplitudes indicated that at least $8 \mathrm{nC}$ of charge per ICMS stimulus phase were needed for the ATs to be discriminated significantly above chance ( $P<0.01$, one-sided binomial test) (Figure 4.8). For catch trials, where no ICMS feedback was delivered for either object, performance was at chance level $(P>0.5)$. 


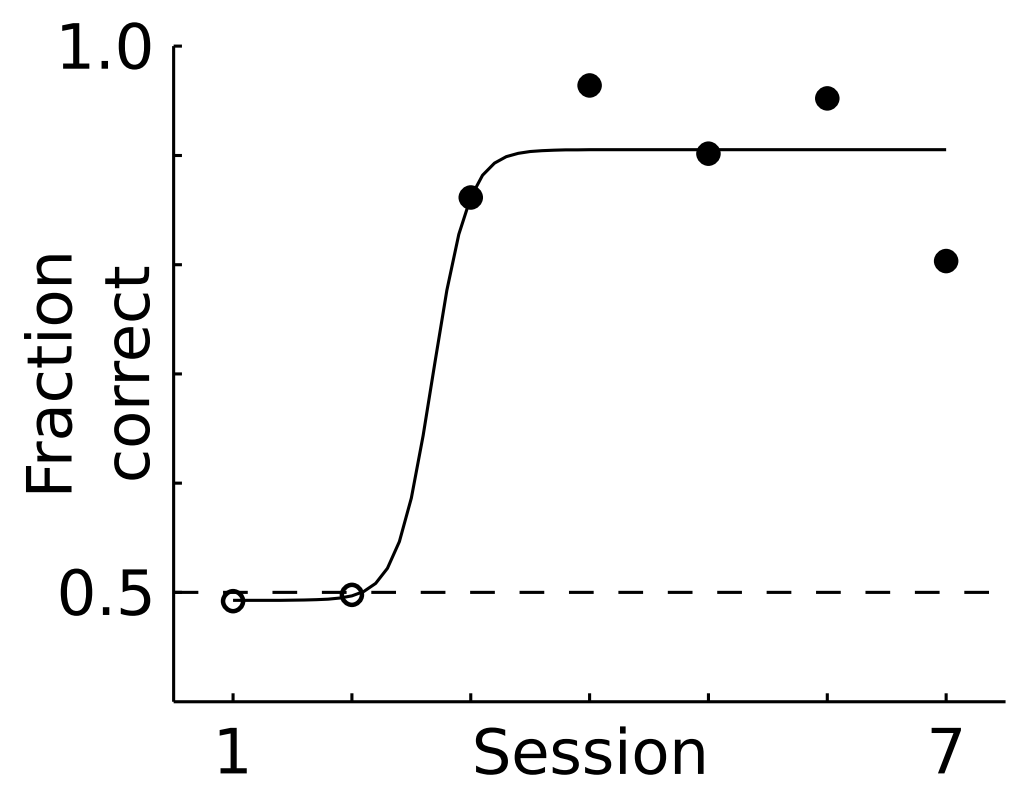

FigURE 4.9: Monkeys rapidly learned vibration feedback. Each circle shows the mean performance of a session for monkey M. Filled circles depict sessions with performance significantly different from chance. All sessions were HC. Curve is sigmoidal line of best fit. Dashed line indicates chance level.

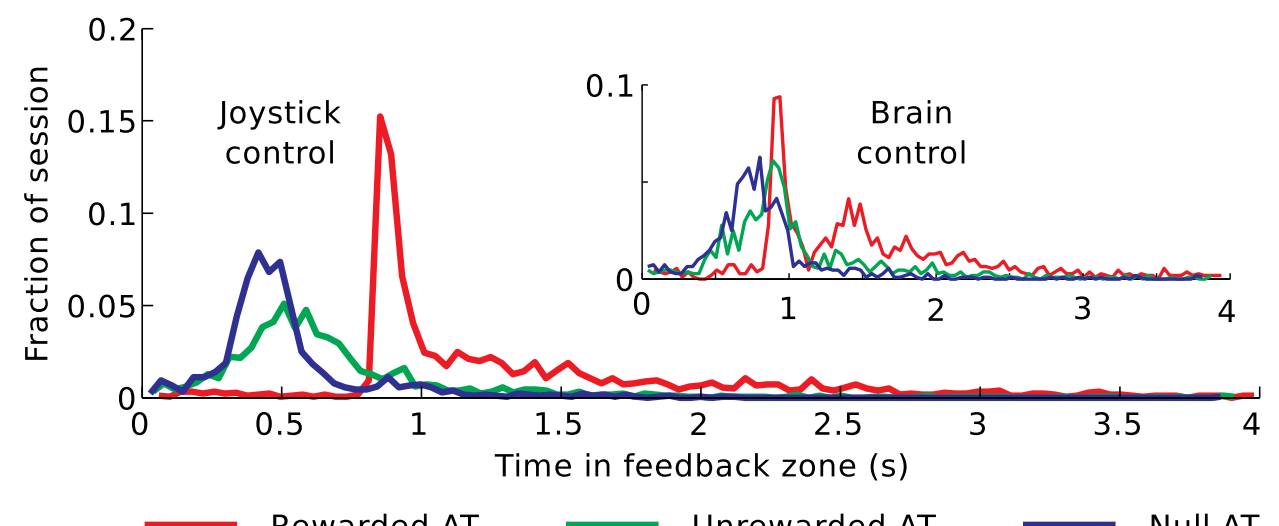

FIGURE 4.10: Feedback exploration histograms. Distributions of times spent exploring each of the three objects for $\mathrm{HC}$ and $\mathrm{BC}$ (insert). 
After learning the object-AT association in Task I, the monkeys continued to show improvements as task complexity increased (Tasks II-V), both in terms of number of correct trials and time spent exploring the feedback zones, (Figure 4.7 on page 69). All of these tasks required active exploration and could not be completed successfully using vision alone. Active exploration of the different ATs was evident from an analysis of object exploration intervals (panel C of Figure 4.7 on page 69). By task design, the monkeys could explore an ICMS feedback zone around each object for several seconds, but were not allowed to stay over the central response zone of the object longer than a hold interval (800 ms in most experiments, see Figure 4.4 on page 67 ). In early sessions, the monkeys spent equal times examining the tactile properties of each object $(P>0.5$, Wilcoxon signed-rank test). Later, the average exploration duration was the shortest for NAT (304 \pm 337 ms; mean \pm std), longer for UAT ( $452 \pm 621 \mathrm{~ms})$ and the longest for RAT (1396 $\pm 942 \mathrm{~ms}$; Figure 4.10 on the preceding page). The differences between these conditions were statistically significant $(P<0.01$, Wilcoxon rank sum test). There was also a tendency over sessions for the exploration duration of NAT and UAT to decrease (see panel C of Figure 4.7 on page 69 ). These results indicate that ICMS feedback operates on a timescale of hundreds of milliseconds, which is comparable to the timescales of detection and discrimination of peripheral tactile stimuli (Lebedev et al., 1994; Liu et al., 2005).

Further evidence of active exploration was evident from the conditional probabilities of selecting an object versus exploring a different one (Figure 4.11 on the following page). The first object touched was selected with high probability (0.7) if it was associated with RAT, but low probabilities (o.o and 0.05, respectively) for NAT and UAT. But after touching the second object, the monkeys had enough information to identify the correct one (either by apprehending it directly or through a process of elimination). The average probability of choosing RAT after NAT or UAT increased from 0.48 to 0.71 , and the probability of transitions from UAT to NAT or vice versa decreased from 0.47 to 0.23 and 0.52 to 0.28 , respectively. This effect was observed for $\mathrm{HC}$ and $\mathrm{BC}$ (black and red fonts, respectively, in 


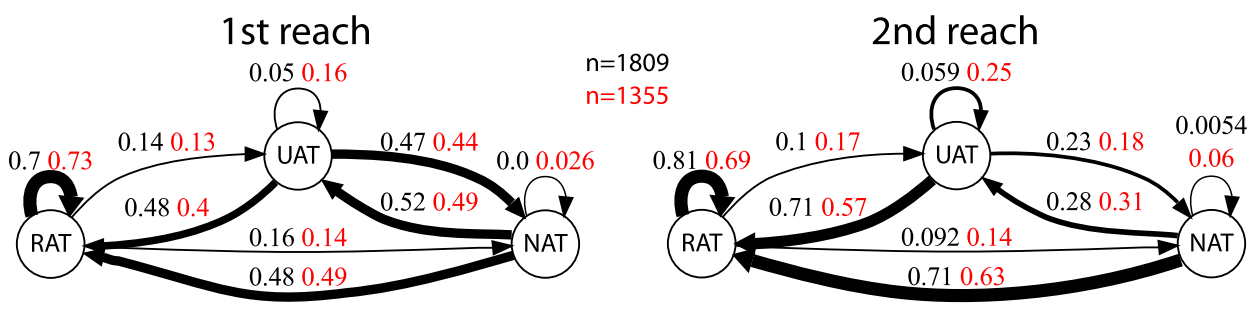

FIGURE 4.11: Exploration probabilities as function of reach number. Diagrams indicate the conditional probabilities of movements toward a RAT, UAT or NAT object given the first (left sub-panel) or second (right sub-panel) reach of a trial. Black labels indicate HC $(n=1809)$ trials; red labels indicate $B C(n=1355)$ trials. Line thickness is proportional to reach probability.

Figure 4.11). These findings indicate that the monkeys retained memory of ICMS patterns similar to the retention of tactile memories that occur during natural touch (Zhou \& Fuster, 1996).

\subsubsection{Brain-machine-brain interface}

Real-time brain control was consistently studied starting with task IV. Although the joystick was disconnected from the control of the avatar, the monkeys were initially permitted to hold the joystick. We call this brain control with hand (BCWH; Lebedev et al., 2005; O'Doherty et al., 2009). BCWH was useful for analysis because it isolated the effects related to simultaneous BMI control and ICMS feedback from those involved in adaptation to brain control without hand (BCWOH; Carmena et al., 2003; Lebedev et al., 2005). In BCWOH sessions, the joystick was removed from the apparatus and the monkeys explored the objects without overt movements of their bodies.

Performance accuracy in BCWH approached that of HC. In monkey M, with more than 200 simultaneously recorded neurons, $73.75 \pm 10.83 \%$ of BC trials were correctly performed (versus $91.48 \pm 4.33 \%$ in $\mathrm{HC}$ ). BCWH accuracy was lower in Monkey N with approximately 50-recorded neurons $(50.37 \pm 9.76 \%$ versus $91.45 \pm 5.73 \%$ in $\mathrm{HC}$; chance level of $33 \%)$. 


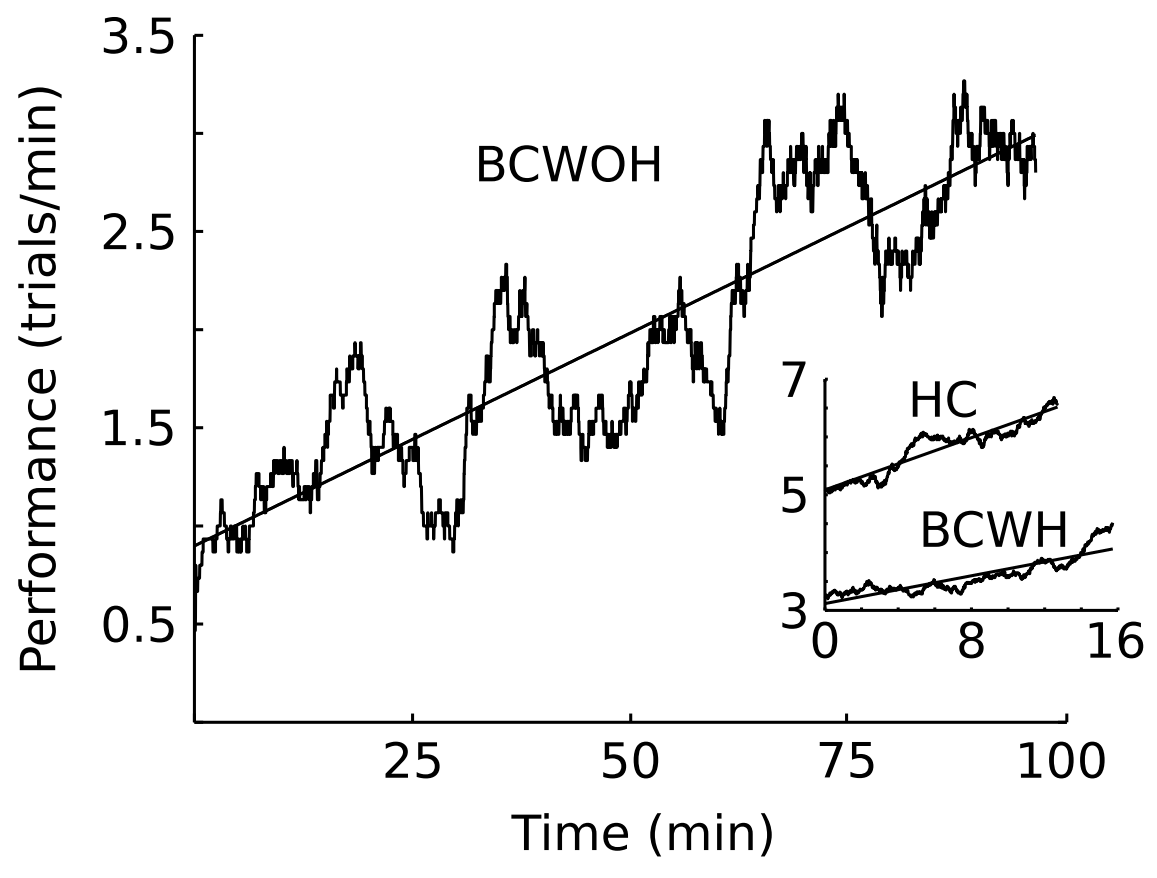

Figure 4.12: Control improves within sessions in hand and brain control. Performance (correct trials/min) is shown as a function of time in a session. Each curve represents averages for BCWOH (main panel, three sessions), HC (insert, 12 sessions) and BCWH (insert, 12 sessions). Lines are significant linear fits $(P<0.001$, F-test for linear regression).

$\mathrm{BCWH}$ reproduced other features of $\mathrm{HC}$, including improvement with training (panel B of Figure 4.7 on page 69), shorter intervals of stay over unrewarded objects (panel C of Figure 4.7 on page 69 and Figure 4.10 on page 71), and the probabilities of transitions between different ATs (red text in panel E of Figure 4.13 on page 77). Intervals spent exploring NAT $(0.834 \pm 0.457 \mathrm{~ms})$ and UAT $(0.862 \pm 0.738 \mathrm{~ms})$ were longer as compared to HC because the monkeys did not move the avatar as quickly. However, the intervals spent exploring NAT and UAT were still significantly shorter as compared to RAT $(P<0.01$ for both, Wilcoxon rank sum test).

Task performance steadily improved within each BCWOH session (Figure 4.12), indicating that the neural ensemble adapted to this control mode. These results demonstrate 
that BMBI control with an ICMS-generated artificial tactile feedback channel was not only comparable to BMI with visual feedback alone (Carmena et al., 2003; Lebedev et al., 2005), but exceeded it in many ways. First, the tasks used here were markedly more difficult as compared to most previous BMI studies where single targets were presented. Additionally, the monkeys held the avatar hand over the objects for approximately $1 \mathrm{~s}$ to probe and select an object, a challenging brain controlled task in and of itself. Such versatility of performance will be required for future clinical neuroprosthetic devices.

\subsubsection{Neurophysiological findings}

The majority of recorded cortical neurons in both monkeys were modulated by arm movements, with modulation patterns retained across different conditions. If the monkey encountered a RAT object, it stopped on the object in the majority of trials, as evident from average position traces (panels B and D of Figure 4.13 on page 77 , left). The accompanying neuronal activity consisted of phasic bursts during the reach. Notably, movements were slower and neuronal bursts more spread out during BC (panels D and E of Figure 4.13 on page 77) as compared to HC (panels B and C of Figure 4.13 on page 77). When encountering an incorrect object (UAT or NAT), an increase in neuronal activity occurred as the monkey moved away (see Figure 4.13 on page 77, right panels).

A variety of directional neuronal tuning patterns were observed during $\mathrm{HC}$ and $\mathrm{BCWH}$. Single neurons exhibited broad tuning curves in which a preferred direction (PD) could be identified in most cases. Practically all possible PDs were represented in the recorded neuronal sample (see Figure 4.14 on page 78 ). Moreover, the PDs remained similar even as the avatar touched different types of objects, including those that evoked ICMS (panels $\mathrm{B}$ and $\mathrm{D}$ of Figure 4.13 on page 77$)$. We believe that the observed stability of neuronal tuning for different ICMS conditions contributed to the accurate performance of the BMBI. Tuning curves shifted after the transition to BC (compare panel A and B of Figure 4.14 on page 78 ), but the PDs were correlated (panel C of Figure 4.14 on page 78 ). A grouping of 
FIGURE 4.13 (following page): M1 modulations during movement to different objects were not disrupted by concurrent ICMS. Each vertical column corresponds to an AT class. A. Schematic of stereotypical reaches made towards the RAT, UAT or NAT objects (left, middle, and right sub-panels, respectively). B. Average movements made in HC towards the objects as in panel A. Reaches are shown along the axis of movement in the direction of the first object picked; only trials with a subsequent reach to counter-clockwise objects are included in the middle and right sub-panels. C. Neuronal modulations for each of the categories in panels A and B; red vertical line is object onset. D. Average movements made in BCWH. Brain controlled avatar movements are shown in red; joystick movements are shown in dashed black. E. Neuronal modulations during BCWH. 
A Rewarded AT Unrewarded AT Null AT
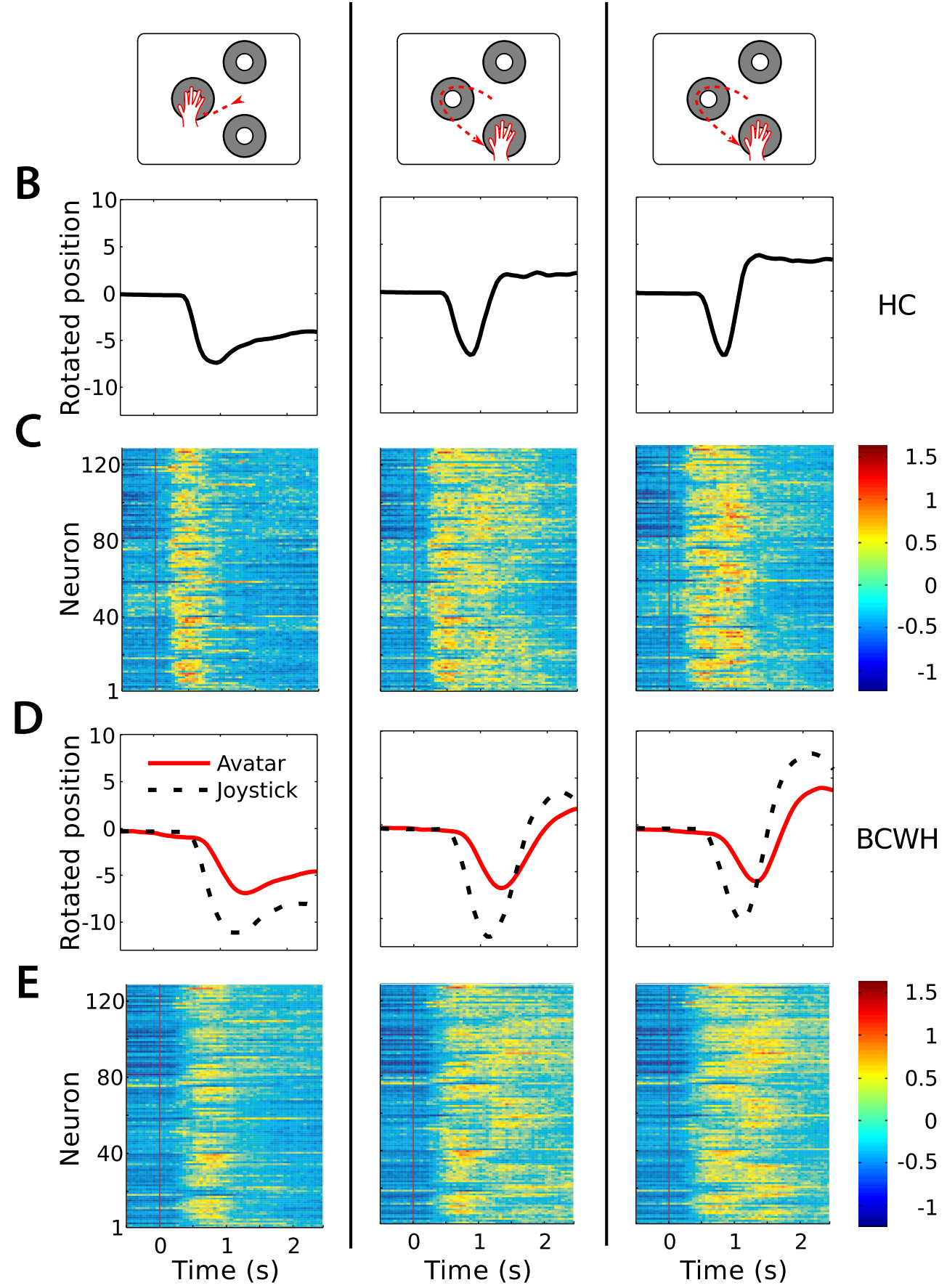


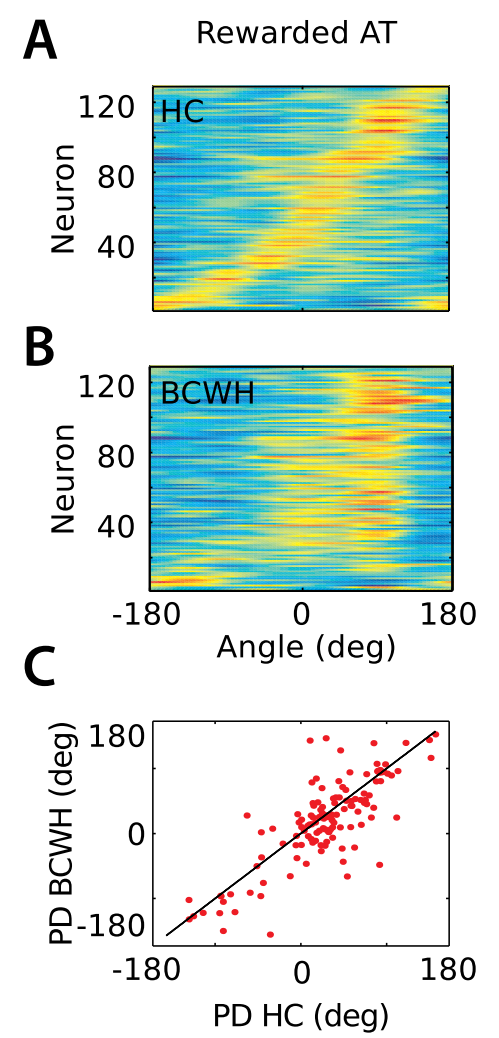

Unrewarded AT
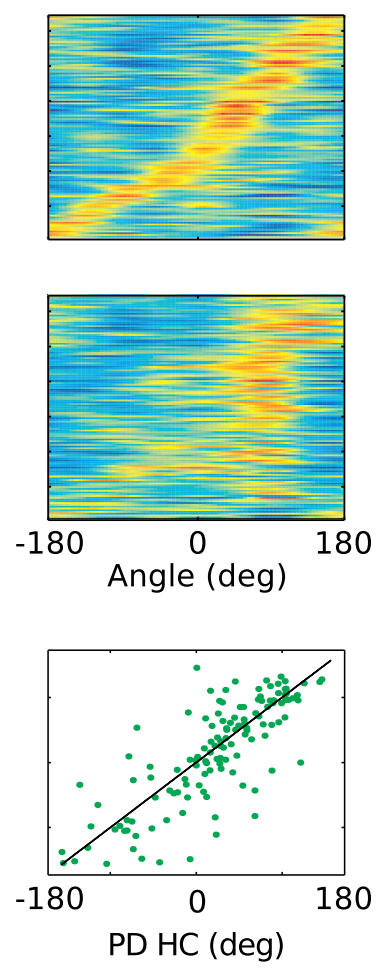

Null AT
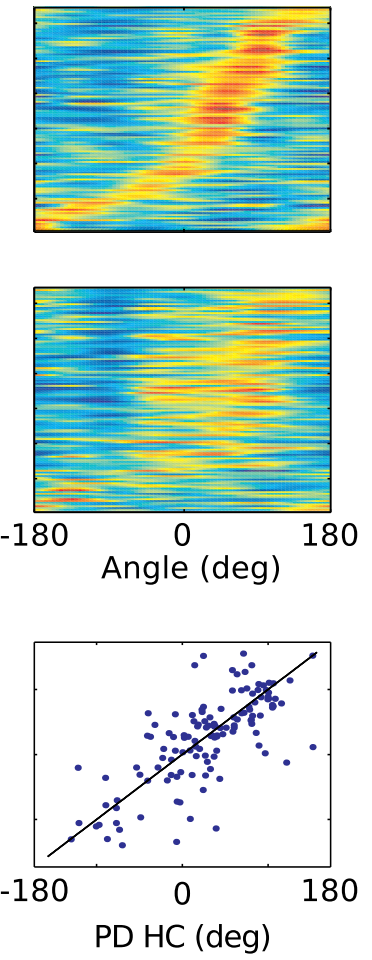

FIGURE 4.14: Tuning adapts during brain control with ICMS feedback. A. Directional tuning for a simultaneously recorded ensemble of Mi neurons for reaches to the RAT, UAT and NAT (left, middle and right sub-panels, respectively) during HC. Neurons are sorted by their PD. B. Directional tuning as in panel A but in BCWH; sorting is the same as in A. C. Comparison of preferred directions in $\mathrm{BC}$ versus $\mathrm{HC}$ for each of the three $\mathrm{AT}$ conditions.

PDs occurred in BCWH-a phenomenon that we reported previously and linked to an increase in correlated bursts of activity in the recorded neurons (Carmena et al., 2003). In $\mathrm{BCWOH}, \mathrm{PD}$ grouping around $90^{\circ}$ was observed in both monkeys (Figure 4.15 on the next page), and sub-populations of neurons exhibited enhanced directional tuning. Thus, volitional control of the BMBI without overt movements of the limbs induced clear and rapid neurophysiological adaptations. 


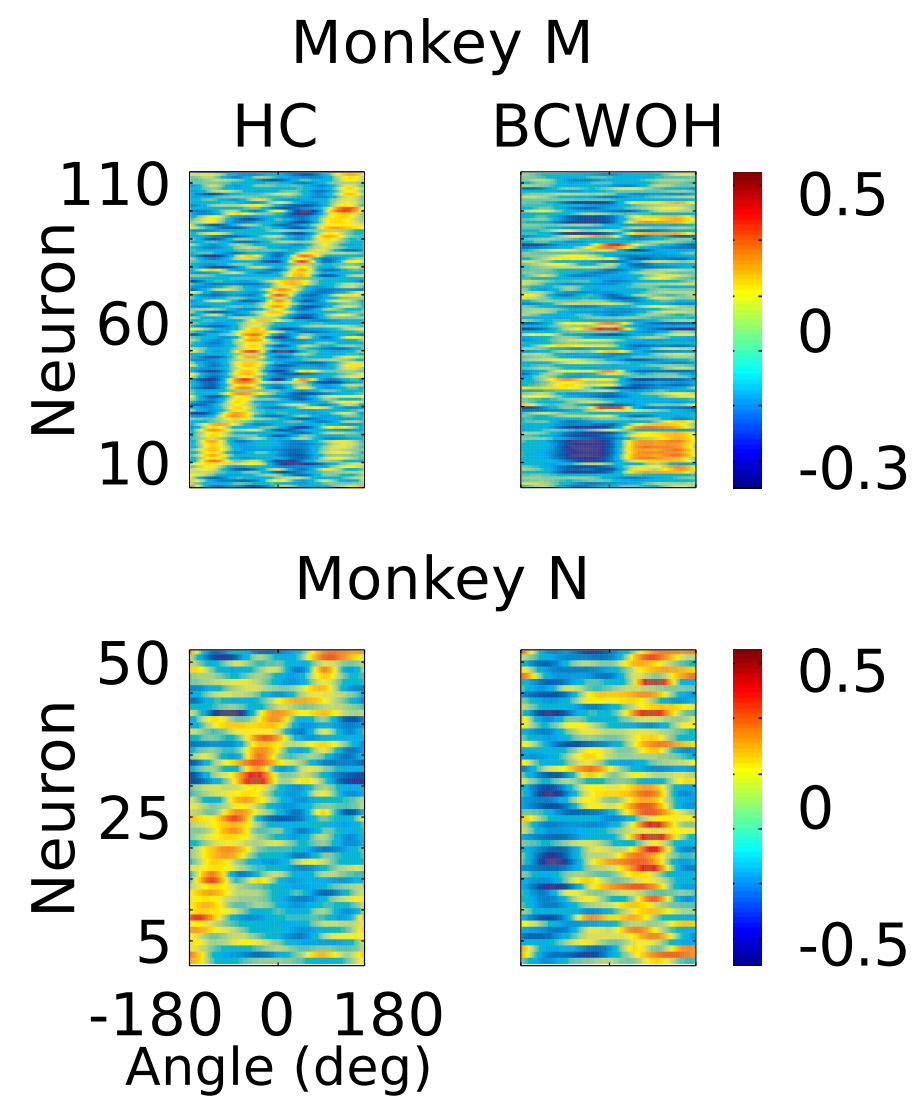

FIGURE 4.15: Grouping of preferred directions occurs in BCWOH. Directional tuning for $\mathrm{HC}$ (left sub-panels) and BCWH (right panels). Data are shown for monkeys $\mathrm{M}$ and $\mathrm{N}$ (upper and lower panels, respectively).

\subsubsection{Avatar incorporation}

We have speculated that long-term operation of a BMI might lead to incorporation of the artificial actuator into the internal cortical representation of the subject's body (Lebedev \& Nicolelis, 2006). We hypothesized that if the brain incorporated the avatar, its movements would evoke cortical activity, even if the avatar were moved for the monkey, rather than by the monkey. This hypothesis was tested in a final experiment in which the monkeys passively observed the avatar search through 3-object sets (task V) driven by a computer reach planner. Reward was delivered whenever the avatar selected a RAT object, assuring that the monkey 

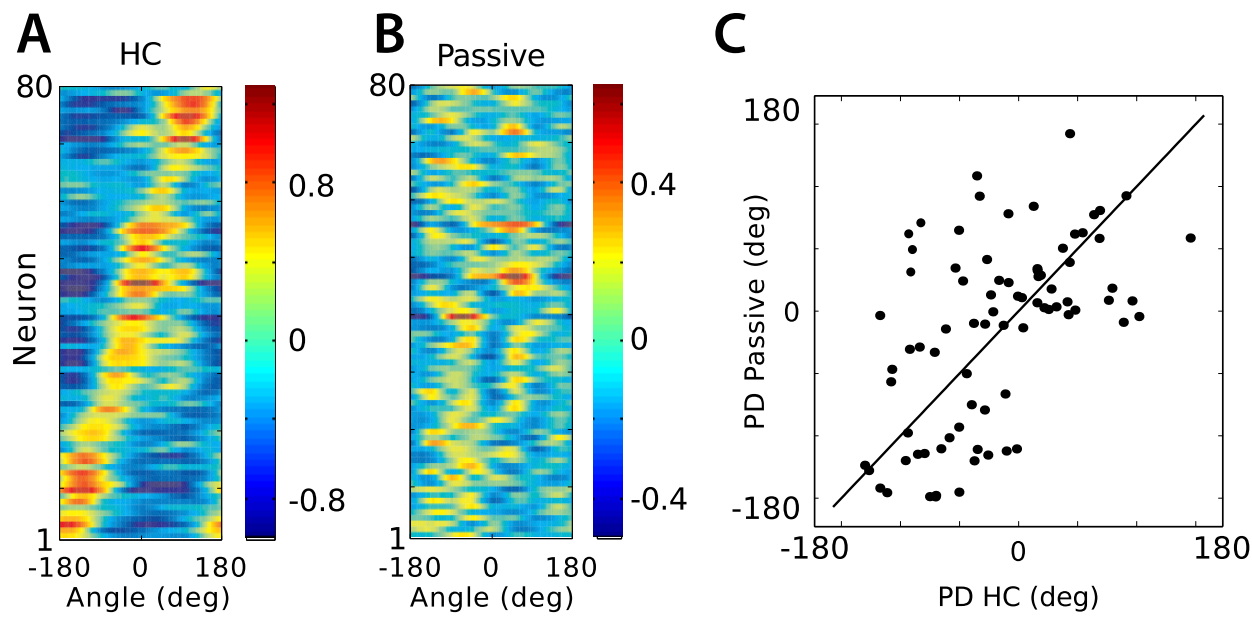

FIgURE 4.16: Passive observation of the avatar elicits Mi firing rate modulations. A. Directional tuning curves to joystick movements. B. Directional tuning curves to passively observed avatar movements. C. Comparison of PDs (angle of peak tuning) for HC versus PDs for passive observation.

attended to the avatar. Directional tuning curves (panels A and B of Figure 4.16) indicated that Mi neurons were tuned to the passive movements of the avatar, albeit with lower tuning depths and bimodal modulations in some neurons. The PDs shifted but remained on average correlated with HC PDs (panel C of Figure 4.16). Moreover, passively observed avatar movements could be predicted from neuronal ensemble activity (panel B of Figure 4.17 on the following page), with similar accuracy to predictions made during $\mathrm{HC}$ (panel A of Figure 4.17 on the next page).

The representation of avatar movements by Mi neurons, even when the avatar was passively observed, supports our suggestion that neuroprosthetic limbs can be incorporated in the brain's internal representation. This suggestion is also supported by a report (Dushanova \& Donoghue, 2010) that identified M1 engagement during a monkey's observation of a human performing a reaching task. 

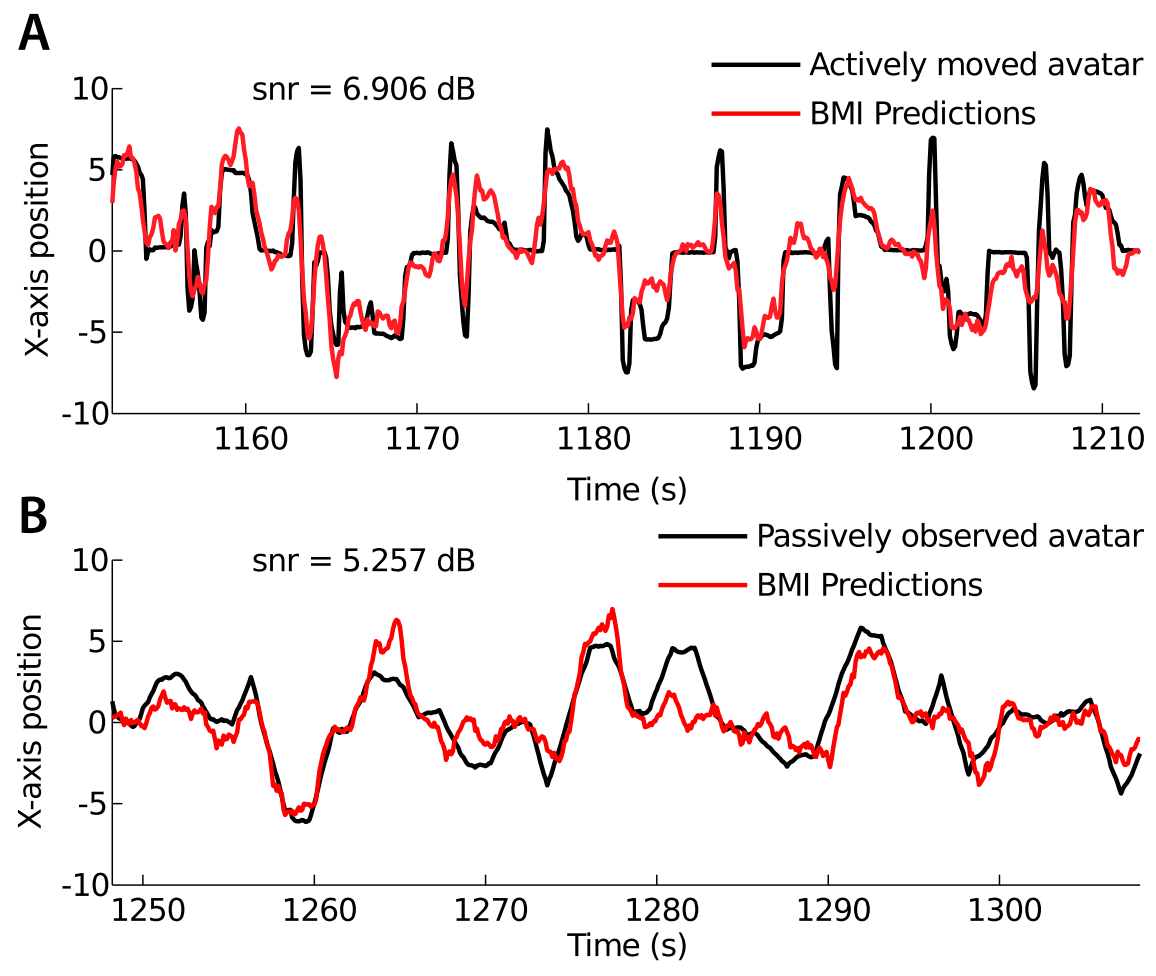

Figure 4.17: Passively observed avatar movements can be predicted from M1 activity. A. Plot of the position of the avatar while the monkey actively controls it (HC) to acquire objects (not shown). Black trace is joystick; red trace is BMI predictions. Signal-to-noise measure of prediction quality is shown in decibels. B. Plot of the position of the avatar while it is being moved with the monkey passively observing; colors as in A.

\subsection{Discussion}

The BMBI developed in this study is the first demonstration of direct bidirectional communication between a non-human primate brain and an external actuator. As both the afferent and efferent communication channels of this BMBI bypassed the monkey's body, we propose that such bidirectional connections between the brain and artificial (mechanical, electronic, robotic, or even virtual) actuators can effectively liberate a brain from the constraints of the body, in both the execution of motor behaviors and the interpretation of somatosensory feedback generated by those acts. 


\subsubsection{Interleaved ICMS and recordings}

One of the bigger technical challenges of this study was the coupling of ICMS feedback with the BMI decoder. Interleaved stimulation and recording intervals were sufficient to provide high-quality input signals for online motor control. In the future, other methods for the stimulation of neural tissue may complement ICMS, for example optogenetic stimulation can excite (Boyden et al., 2005) or inhibit (Zhang et al., 2007) specific neural structures while producing little or no artifact in recording channels. Still, chronic electrical stimulation of the nervous system has a long track record of safety and efficacy in animals and humans, a property not fully documented for optogenetics.

\subsubsection{Implementation in human subjects}

While the monkeys in this study were used as models of amputee or spinal cord injured patients, they had intact limbs and cortical structures with normal connectivity and somatotopy. A candidate recipient of a clinical neuroprosthetic limb would possess a sensorimotor cortex exhibiting profound plastic changes (Pons et al., 1991). However, the sensory cortex recovers a somewhat typical organization after allogeneic hand transplantation (Frey et al., 2008), presumably due to renewed sensory input. It is possible that ICMS of S1, congruent with active touch using a neuroprosthesis, could result in similar recovery of the cortical map for the users of a BMBI.

\subsubsection{Prosthesis incorporation}

Any practical neuroprostheses should act and feel as the subject's natural limbs (Lebedev \& Nicolelis, 2006). We suggest that concurrent sensory and motor loops could be used to gradually incorporate a neuroprosthetic limb into a human subject's internal representation. Taking this idea even further, we suggest that future applications of BMBIs will not be limited to limb prostheses, but may include a range of devices designed for reciprocal communication between neural structures and a variety of external devices or other neural structures. In 
these applications, ICMS may substitute for normal sensations or even represent entirely new sensory modalities. 


\section{Randomly patterned ICMS}

\subsection{Abstract}

NTracortical microstimulation (ICMS) has promise as a means for delivering so-
matosensory feedback in neuroprosthetic systems. Various tactile sensations could be encoded by temporal, spatial, or spatiotemporal patterns of ICMS. However, the sensitivity of subjects to sensations encoded by precise temporal patterns of ICMS is unknown, as is how different two ICMS patterns must be before a subject can reliably discriminate them. We trained rhesus monkeys in an active-touch task in which they discriminated periodic pulse-trains of ICMS (200 Hz bursts at a $10 \mathrm{~Hz}$ secondary frequency) from pulse-trains with distorted periodicity (200 Hz bursts at a variable instantaneous secondary frequency). The statistics of the aperiodic pulse trains were drawn from a gamma distribution with equal mean inter-burst intervals to the periodic pulse trains. The monkeys distinguished periodic pulse trains from aperiodic pulse trains with coefficients of variation of 0.25 or greater. Reconstruction of movement kinematics, extracted from the activity of sensorimotor neuronal populations recorded concurrently with the delivery of ICMS feedback, improved when the recording intervals affected by ICMS artifacts were removed from analysis. These results 
add to the growing evidence that temporally patterned ICMS can be used to encode texture for neuroprosthetic devices.

\subsection{Introduction}

Sensory neuroprostheses and sensory substitution systems for the restoration of hearing (Merzenich et al., 1974; Fallon et al., 2008) and vision (Bach-y-Rita et al., 1969, 1998; Bach-y-Rita \& Kercel, 2003; Dobelle et al., 1974; Dagnelie, 2008; Cohen, 2007) have been investigated for several decades. Interest in neuroprosthetic devices that combine both motor and sensory components has developed more recently (O'Doherty et al., 2009; Mussa-Ivaldi et al., 2010; Fagg et al., 2009; Marzullo et al., 2010; Suminski et al., 2010; Venkatraman \& Carmena, 2011). One example of a bidirectional neuroprosthesis is a robotic limb controlled by brain activity while sensory information from prosthetic sensors is delivered to somatosensory areas of the brain (Lebedev \& Nicolelis, 2006; Nicolelis \& Lebedev, 2009). Other possible implementations include sensorized neuroprostheses for restoration of bipedal walking (Fitzsimmons et al., 2009) and putative systems that combine both speech production (Guenther et al., 2009; Brumberg et al., 2010) and hearing (Merzenich et al., 1974; Fallon et al., 2008; Peterson et al., 2010).

During the last several years we have been studying intracortical microstimulation (ICMS) delivered through microelectrode arrays chronically implanted in the primary somatosensory cortex $\left(\mathrm{S}_{1}\right)$ as a means of adding a somatosensory feedback loop to a brainmachine interface (BMI; Fitzsimmons et al., 2007; O’Doherty et al., 2009). Taken together with previous work showing that primates (Romo et al., 1998, 2000; de Lafuente \& Romo, 2005) and rodents (Butovas \& Schwarz, 2007; Houweling \& Brecht, 2008; Talwar et al., 2002) can discriminate ICMS patterns, these results support the suggestion that ICMS of S1 could equip neuroprosthetic limbs with the sense of touch.

We envision future limb neuroprosthetic devices as BMI-operated robotic arms that are 
equipped with touch sensors (O’Doherty et al., 2009; Lebedev \& Nicolelis, 2006; Nicolelis \& Lebedev, 2009). The sensors detect instances when the arm interacts with external objects send signals to the brain in the form of ICMS. We have suggested that long-term operation of such system, that we call a brain-machine-brain interface (BMBI), could result in the incorporation of the prosthesis into the brain's representation of the body, so that the artificial limb starts to act and feel as the subject's own (Lebedev \& Nicolelis, 2006). Notwithstanding initial encouraging results (O’Doherty et al., 2009; see also Chapter 3 and Chapter 4), it is unclear whether ICMS would be sufficient to reproduce the rich sensory information of the world of touch (Katz \& Krueger, 1989).

In particular, it is not well understood what kinds of ICMS patterns are optimal for active touch. Previously, my colleagues and I have shown that both New World (Fitzsimmons et al., 2007) and Old World monkeys (see Chapter 4) can discriminate temporal ICMS patterns applied to $\mathrm{S}_{1}$ that consist of short $(50-300 \mathrm{~ms})$ high-frequency $(100-400 \mathrm{~Hz})$ pulse-trains presented at a lower secondary frequency $(2-10 \mathrm{~Hz})$. These patterns of ICMS could, in principle, represent a wide variety of tactile inputs, especially when combined with spatial encoding (Fitzsimmons et al., 2007). Modulations of sensory inputs in this frequency range correspond to the sensation of flutter (Mountcastle et al., 1990; Lebedev et al., 1994; Salinas et al., 2000). These timescales are also similar to neuronal modulations involved in texture encoding in the somatosensory system (Phillips \& Johnson, 1981; Sinclair \& Burton, 1991; Sinclair et al., 1996; Gamzu \& Ahissar, 2001), which makes these ICMS patterns particularly worthy candidates for exploration.

In this study, we systematically examined the ability of rhesus monkeys to discriminate a range of temporal ICMS patterns applied to S1. We sought to determine whether rhesus monkeys could discriminate aperiodic ICMS pulse-trains that were derived from a strictly periodic pulse-train by an introduction of randomness to the ICMS secondary frequency. The degree of randomness was varied from trial to trial, which allowed us to quantify the monkeys' sensitivity to ICMS frequency modulations. Concurrently with ICMS delivery, we recorded 
from large populations of cortical neurons using multielectrode implants. Kinematics of reach movements were extracted from this large-scale activity offline to estimate the accuracy of a BMBI with a somatosensory feedback loop that transmits aperiodic ICMS patterns.

\subsection{Methods}

\subsubsection{Implants}

The experiments were conducted in two rhesus monkeys ( $\mathrm{M}$ and $\mathrm{N}$, male and female, respectively). Each was chronically implanted with multi-electrode arrays in several cortical areas following our implantation methods (Nicolelis et al., 2003; Carmena et al., 2003, see also Chapter 2). The same electrode array design was used for both large-scale neural recordings and ICMS delivery (O’Doherty et al., 2009). Each monkey received four 96channel microelectrode arrays placed in the arm and leg representation areas of $\mathrm{S}_{1}$ (panel A of Figure 4.2 on page 65 ). Only data from the arrays implanted in the left upper-limb representation area are reported here. For further details of array topologies see Chapter 2.

\subsubsection{Behavioral task}

The monkeys were trained in a reaching task in which they manipulated a hand-led joystick to move a virtual reality arm (avatar) displayed on a computer screen (Figure 5.1 on the next page). Both monkeys performed the task with their left hands. Monkeys reached with the avatar arm towards screen objects and searched for an object with a particular artificial texture indicated by ICMS of S1. The objects were circular in shape and visually identical. Each monkey was previously trained in other several variants of this task. In this study, the monkeys were shown two objects, one of which was associated with a periodic ICMS pattern and the other with an aperiodic pattern (Figure 5.1 on the following page and Figure 5.2 on page 89). The monkeys were rewarded for selecting the object paired with periodic ICMS. The objects appeared at different locations on the screen, with the constraint that the distance from the screen center to each object was fixed, and the angular distance between the objects 

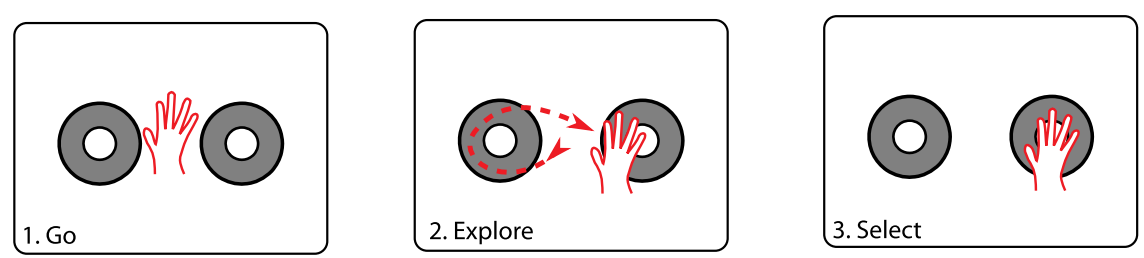

FIGURE 5.1: Aperiodic task structure. Monkeys initiated a trial by holding the avatar in the center of the screen until two peripheral objects appeared (500 to $1300 \mathrm{~ms}$, random per trial; left panel). Next, the monkeys freely explored the objects (middle panel). Finally, an object was selected by holding the avatar within the response zone for $2000 \mathrm{~ms}$ (right panel).

was $180^{\circ}$ (i.e. $\mathrm{C}_{2}$ rotationally symmetrical). If the correct object was selected, the monkeys were rewarded with a drop of fruit juice.

Each trial commenced when a monkey grasped the joystick with its left hand. At this point, a circular target appeared in the center of the screen. The monkey placed the avatar arm on that center target for 500 to $1300 \mathrm{~ms}$. Then, the central target disappeared and two peripheral objects appeared. Each consisted of a central response zone and a peripheral feedback zone (See Figure 5.2 on the following page). Once the avatar hand entered the feedback zone, ICMS pulse trains were delivered to electrode pairs in S1.

ICMS was delivered to different parts of $S_{1}$ for each monkey. For monkey $M$, the hand representation area of $S_{1}$ was used as the target for ICMS, so that the monkey experienced putative sensations in its hand (panel B of Figure 4.2 on page 65 ). For monkey N, ICMS was applied to the thigh representation area of $S_{1}$. The monkeys were permitted to explore the virtual objects in any sequence, but they were not allowed to overstay at the incorrect object longer than the hold period of $2 \mathrm{~s}$. A trial was concluded with a reward when the monkey placed the avatar hand within the response zone of the correct object for $2 \mathrm{~s}$; no reward was delivered if the object was incorrect. The next trial commenced after an inter-trial interval of 0.5 s. Trial structure is illustrated in Figure 5.1. 
A B

Periodic ICMS

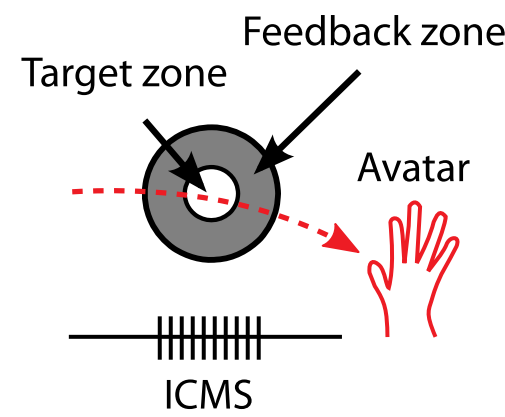

Aperiodic ICMS

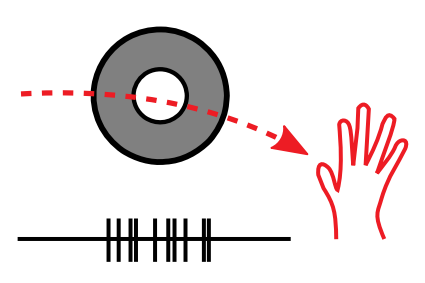

FIGURE 5.2: Aperiodic ICMS stimulation paradigm. Objects on the screen consisted of a central response zone surrounded by a feedback zone. Movement of the avatar though the feedback or response zone caused the delivery of ICMS pulse trains. A. Periodic ICMS. B. Aperiodic ICMS.

\subsubsection{ICMS patterns}

ICMS trains consisted of symmetric, biphasic, charge-balanced pulses of ICMS delivered in a bipolar fashion through adjacent pairs of microwires (Fitzsimmons et al., 2007; O'Doherty et al., 2009). For monkey $M$, the electrode pairs chosen for stimulation had receptive fields in the digits of the hand and the upper arm (panel B of Figure 4.1 on page 60). For Monkey $\mathrm{N}$, the tactile receptive fields in the upper limb implant were indistinct, therefore the electrode pair used for stimulation chosen from the electrodes in the lower limb implant, and accordingly had a sensory receptive field localized to the leg. For monkey M, the anodic and cathodic phases of stimulation each had amplitudes of $150 \mu \mathrm{A}$ and pulse widths of $105 \mu \mathrm{s}$; for monkey N, $150 \mu \mathrm{A}$ and $200 \mu$ s, respectively. The anodic and cathodic waveforms were separated by $25 \mu \mathrm{s}$.

The temporal pattern of ICMS consisted of $200 \mathrm{~Hz}$ pulse trains delivered for $50 \mathrm{~ms}$ and presented at a lower secondary frequency. The secondary frequency for the rewarded 
A

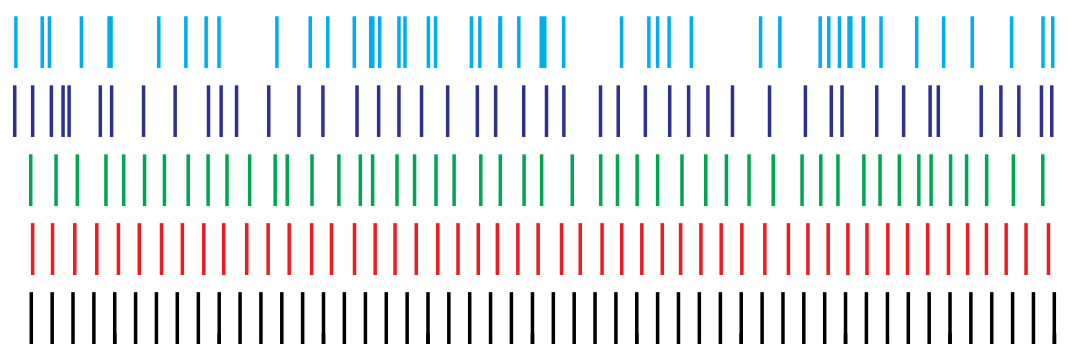

B

$500 \mathrm{~ms}$

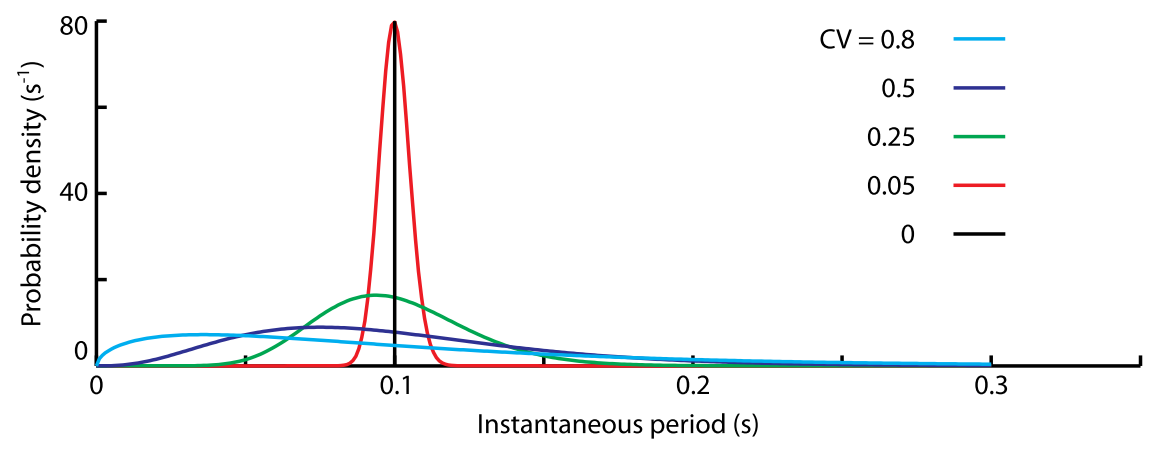

Figure 5.3: Example aperiodic ICMS pulse trains and distributions. A. Raster indicates the range of variability of inter-burst intervals $(C V=0$, black; $C V=0.05$, red; $C V=0.25$, green; $C V=0.5$, blue; $C V=0.8$, cyan). Note that each vertical line indicates a brief burst of ICMS, not a single pulse. B. Distributions of inter-burst intervals corresponding with the rasters shown in panel $\mathrm{A}$.

artificial texture was a constant $10 \mathrm{~Hz}$. For the unrewarded textures, the timing of the ICMS bursts was aperiodic. The interval between each aperiodic burst was a random variable drawn from a gamma distribution of instantaneous inter-burst intervals (similar to Dorval et al., 2010):

$$
f(x ; k, \theta)=x^{k-1} \frac{e^{-x / \theta}}{\theta^{k} \Gamma(k)} \quad \text { for } x \geq 0 \text { and } k, \theta>0,
$$

where $f$ is the probability density function, $x$ is the inter-burst interval, $k$ is the shape parameter, $\theta$ is the scale parameter, and $\Gamma$ is the gamma function. We computed the shape 
and scale parameters as a function of the mean inter-burst interval, $\mu$, and the coefficient of variation, $\mathrm{CV}$, the ratio of the standard deviation to the mean:

$$
\begin{aligned}
& k=\frac{1}{C V^{2}} \\
& \theta=C V^{2} \mu .
\end{aligned}
$$

This allowed the construction of aperiodic pulse trains with inter-burst intervals equal in expectation to the periodic pulse trains while giving control over the degree of aperiodicity: the higher the $\mathrm{CV}$, the more random the pulse-train. A pulse train with a $\mathrm{CV}$ of zero would be equivalent to the periodic, rewarded pattern. The average number of ICMS pulses per unit time was the same, in expectation, for the periodic and aperiodic patterns. Examples of pulse trains with different CVs are shown in panel A of Figure 5.3 on the preceding page and the distributions they were drawn from are shown in panel B of Figure 5.3 on the previous page.

\subsubsection{Kinematics extraction and artifact suppression}

An important question arising from the use of ICMS for sensory feedback is whether the stimulation causes artifacts in cortical neural ensemble recordings and how these artifacts can be dealt with to minimize their impact on BMI operations. To address this, we processed the neural recordings by removing (blanking) different amounts of neural activity immediately subsequent to each pulse of ICMS and then performed decoding with the processed data. By systematically varying the length of the blanking intervals we could determine the amount of artifact removal that produced the most accurate movement reconstructions.

Artifact removal was implemented as follows. The stimulation artifacts had stereotypical shapes when recorded by the spike acquisition system, and we could reliably detect artifacts by using spike-sorting templates that matched the artifact shapes, allowing us to determine the precise time of each stimulation pulse by its artifact. We then could ignore spiking on 


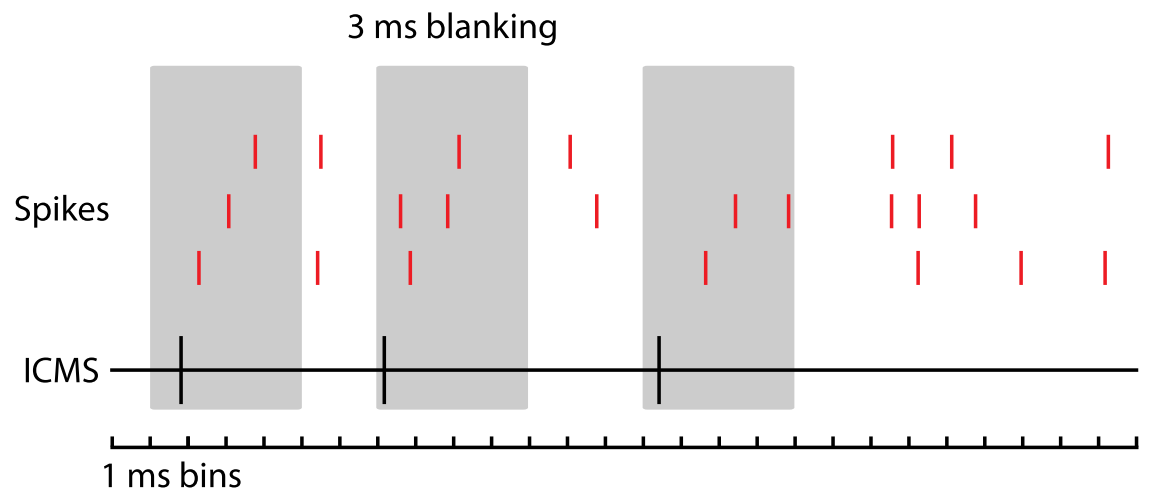

FIGURE 5.4: Schematic of the blanking procedure. Spikes and ICMS pulses were categorized into $1 \mathrm{~ms}$ bins. For each bin containing an ICMS pulse, it and a variable number of subsequent bins (three shown here) were blanked.

every channel for a variable number of milliseconds, $x$, after each stimulation pulse. For the analysis here, we blanked from 0 to $10 \mathrm{~ms}$, in integer steps:

$$
x \in \mathbb{Z}_{10}
$$

This was done by first counting spikes in $1 \mathrm{~ms}$ non-overlapping time windows (i.e. binning at $1 \mathrm{~ms}$ resolution). We then zeroed the spike counts in bins that were equal to or less than $\mathrm{x}$ ms after the stimulation pulse. For example, for $x=3$, we zeroed the bins at times $t$, $t+1, t+2$, and $t+3$, where $t$ is the $1 \mathrm{~ms}$ bin of the stimulation pulse (see Figure 5.5). For $x=0$, we zeroed the bin at the stimulation pulse only.

Then, we summed adjacent bins to produce spike counts in $100 \mathrm{~ms}$ non-overlapping bins for the decoders. For this last step, we adjusted the spike counts by multiplying by the quantity:

$$
\frac{100}{100-n_{\text {blanked }}}
$$

where $n_{\text {blanked }}$ is the number of zeroed $1 \mathrm{~ms}$ bins in the $100 \mathrm{~ms}$ bin. This operation pre- 
serves, in expectation, the number of spikes in each $100 \mathrm{~ms}$ bin, by performing extrapolation.

The $\mathrm{X}$ and $\mathrm{Y}$ position of the avatar was extracted from cortical activity using a 5 th order unscented Kalman filter (UKF; Li et al., 2009) and Wiener filter (WF; Carmena et al., 2003). See also Chapter 2 for further details.

For both algorithms, we evaluated the decoding accuracy after artifact blanking. We performed 2-fold cross-validation with each algorithm on 26 sessions, 13 from each monkey. For the UKF, we used a tuning model with linear weights for position, velocity, distance from center of workspace, and magnitude of velocity. The UKF had 3 future taps, 2 past taps, and one tap in the movement model (see Li et al., 2009, for further details). The tuning model weights were fit with adaptive ridge regression (Grandvalet, 1998), with the ridge parameter found by cross-validation on the training data. For the WF, we used 10 taps of spiking history and predicted the position only. The Wiener coefficients were fit using ridge regression with the ridge parameter found by cross-validation on the training data.

\subsection{Results}

\subsubsection{Learning}

Initially, both monkeys were required to discriminate between the periodic (rewarded) ICMS pattern and an aperiodic pattern with a CV of o.8. Each monkey learned this discrimination task in approximately 8 days (Figure 5.5 on the following page). Monkey $\mathrm{N}$ stabilized at a

performance level of approximately 90\% correctly executed trials, monkey $\mathrm{M}$ at an 85\% level. These learning curves are consistent with our previous results on rhesus-monkey learning in ICMS-instructed tasks (O’Doherty et al., 2009, see also Chapter 3).

\subsubsection{Psychometrics}

After both monkeys learned to discriminate periodic ICMS from aperiodic with a CV of o.8, we began behavioral sessions in which the CV of the aperiodic ICMS pattern varied on every trial. In these sessions, the distribution of CVs was picked so that for half of the trials 


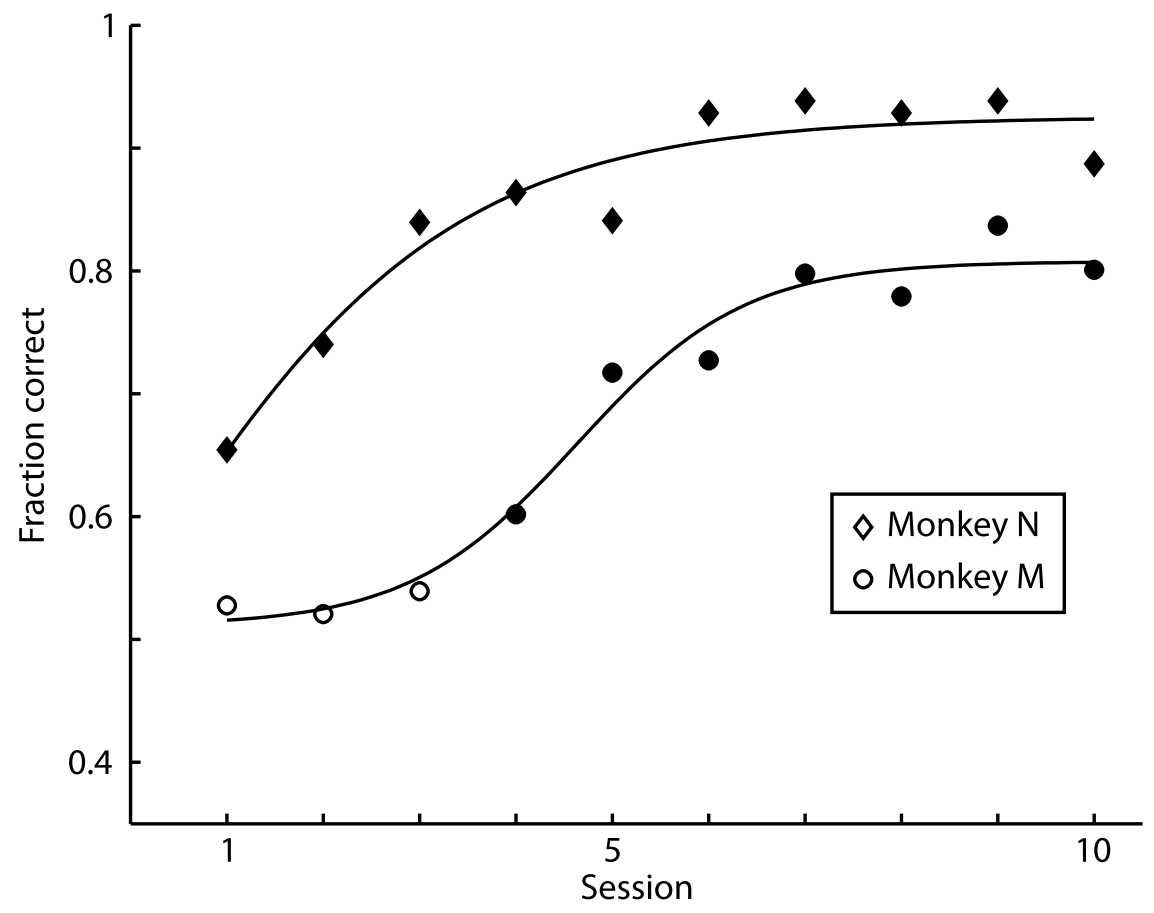

FIGURE 5.5: Learning to discriminate regular and irregular ICMS pulse trains. Summary of the behavioral performance of monkeys $\mathrm{M}$ (circles) and $\mathrm{N}$ (diamonds) for 10 sessions as they learned the task. Each symbol shows the mean performance for the session. Filled symbols depict sessions with performance significantly different from chance $(P<0.05$, one-sided binomial test). Curves are the sigmoidal lines of best fit.

the $\mathrm{CV}$ of the unrewarded object was greater or equal to o.6. These sessions continued for two weeks, yielding a database for psychometric analysis.

Psychometric curves (i.e., graphs showing the proportion of correctly performed trials as the function of CV, Figure 5.6 on the next page) indicated a clear dependency of discrimination accuracy on the degree of randomness of the comparison ICMS pattern. Performance stabilized for CVs higher than 0.8 and gradually decreased for the CV values lower than that value. The threshold CV for discrimination for both monkeys was 0.25. Below this value, the monkeys performed at chance levels.

The discrimination of ICMS patterns was performed through active exploration of the 

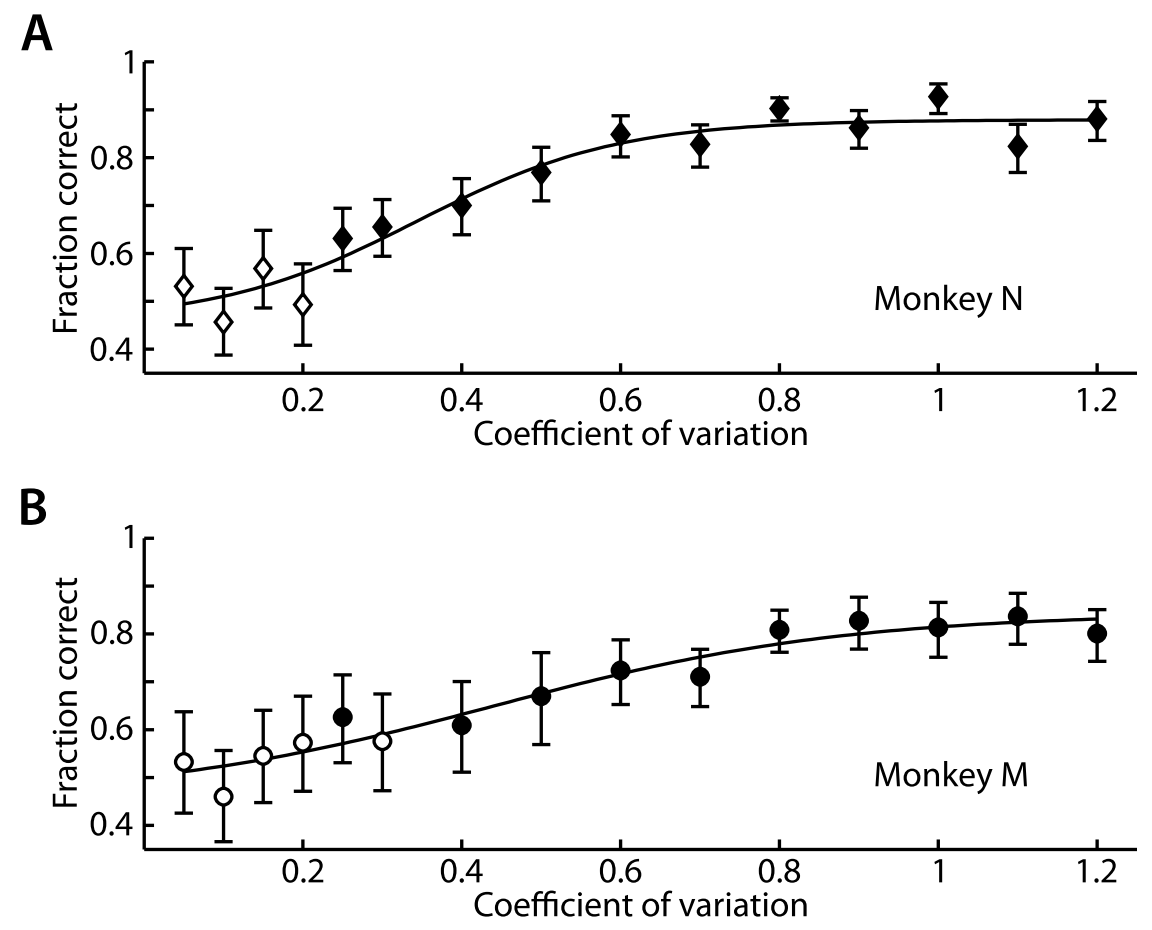

FIGURE 5.6: Psychometric curves for different coefficients of variation. A. Mean performance at differentiating periodic versus aperiodic ICMS pulse trains as a function of CV for monkey $\mathrm{N}$. Each symbol represents the mean performance across sessions; error bars indicate 95\% confidence intervals. Curves are the sigmoidal lines of best fit. Symbols are as in Figure 5.5. B. Same as panel A, but for monkey $\mathrm{M}$.

objects: a monkey would apprehend the feedback zone of the object with the avatar to acquire the artificial texture and then either select that object if it perceived the ICMS pattern as periodic and leave if the pattern was perceived as aperiodic. This active exploration was evident from an analysis of object exploration intervals (panels A and B of Figure 5.7 on the following page). For very low CVs, the statistics of visit durations were the same for periodic and aperiodic patterns (panel A of Figure 5.7 on the next page). The distribution of these intervals indicated short (less than $2 \mathrm{~s}$ ), exploratory visits and a prominent peak at $2 \mathrm{~s}$ that corresponded to the selecting the object. Both the exploratory part of the distribution and the peaks were the same for periodic and weakly aperiodic (e.g. CV of 0.05) ICMS (panel A 


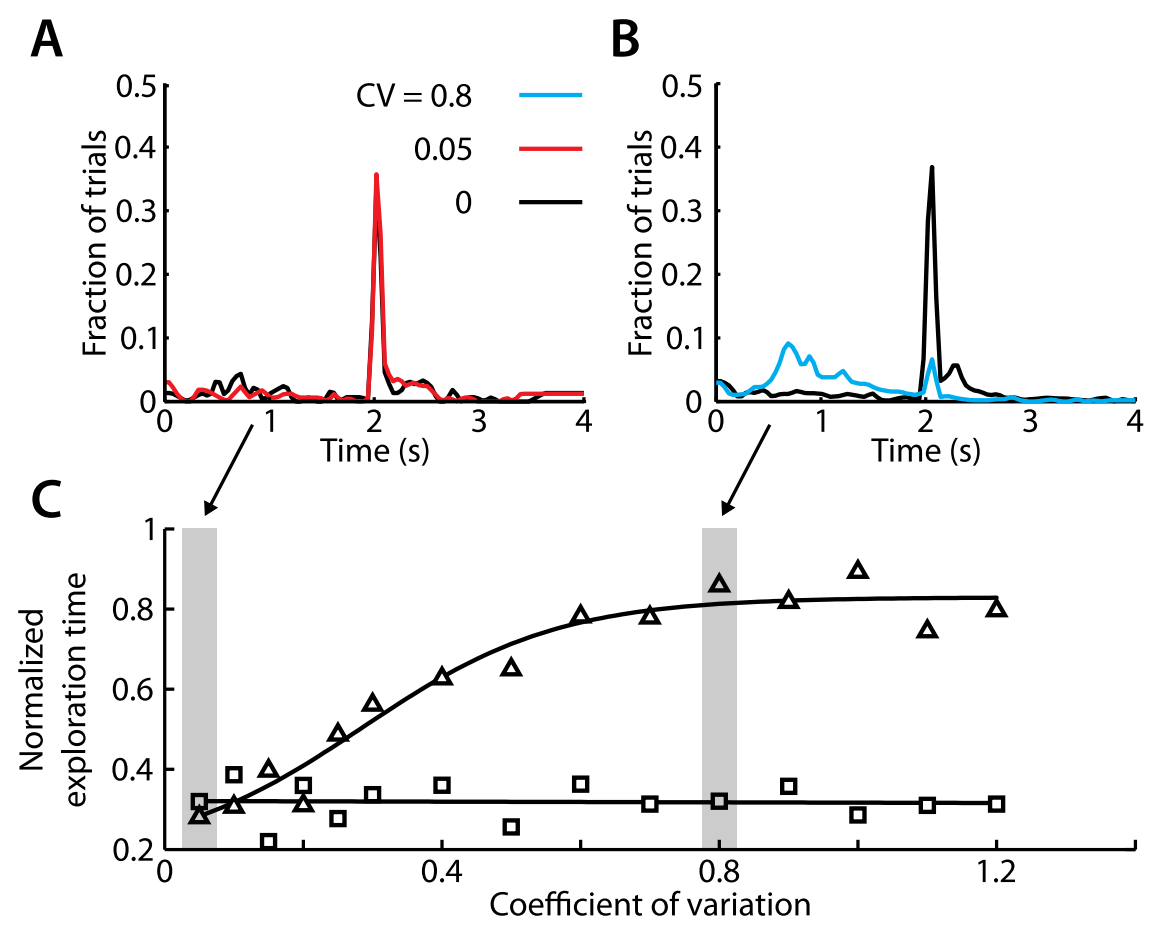

Figure 5.7: Time spent exploring the virtual objects as a function of CV. A. Histograms of the fraction of trials spent exploring the rewarded object $(C V=0$, black trace) versus the unrewarded object $(C V=0.05$, red trace) for trials with that $C V$ combination. B. Same as panel A but for trials unrewarded objects associated with aperiodic ICMS trains with a CV of 0.8 (cyan trace). C. Summary of the normalized exploration times for the rewarded object (square symbols) versus the unrewarded object (triangular symbols) as a function of CV. Gray shaded zones correspond to the data in panels A and B.

of Figure 5.7, compare black and red lines). The statistics were markedly different for higher CVs (panel B of Figure 5.7). The distribution of visit durations for aperiodic ICMS with a CV of o.8 (cyan line) revealed the predominance of exploratory visits with an average duration of $0.8 \mathrm{~s}$ and a small peak at the hold time of $2 \mathrm{~s}$. For the periodic pattern (black line), the distribution only showed the large peak at $2 \mathrm{~s}$. Note a small number of visits with the duration longer than $2 \mathrm{~s}$. They correspond to the cases in which the avatar stayed for some time in the feedback zone (where there was no limit on stay duration) before entering the response zone. These data indicate that it took the monkey on average $0.8 \mathrm{~s}$ to recognize 
the random pattern, and to switch to the correct object associated with the periodic ICMS pattern.

The changes in monkey exploratory behavior for patterns with different degrees of randomness are clear from an analysis of exploration times (object visits shorter than $2 \mathrm{~s}$ ) expressed as the percentage of the total time spent apprehending the object (all visits, panel $\mathrm{C}$ of Figure 5.7 on the preceding page). When the monkey touched an object associated with an aperiodic pattern (panel C of Figure 5.7 on the previous page, triangles), it tended to make more exploratory visits than when the monkey touched an object associated with a periodic pattern (squares). For high CVs (CV greater than 0.7$)$, the duration of exploratory visits constituted approximately 0.8 of the total duration of visits. For lower CVs, this value decreased, indicating that the monkey more often made a decision to stay on the object.

\subsubsection{Kinematics extraction}

Figure 5.8 on the following page shows the average accuracy for the extraction of avatar position from cortical ensemble activity for different lengths of artifact blanking intervals. Consistent with our previous results ( $\mathrm{Li}$ et al., 2009), the unscented Kalman filter outperformed the Wiener filter. For monkey M, the peak accuracy was $5.4 \pm 0.17 \mathrm{~dB}$ (mean \pm standard error) for the UKF and $4.0 \pm 0.14 \mathrm{~dB}$ for the WF. For monkey $\mathrm{N}$, these values were $2.9 \pm 0.16 \mathrm{~dB}$ and $2.5 \pm 0.14 \mathrm{~dB}$, respectively. These accuracy values are within the range typically observed for BMI predictions (Fitzsimmons et al., 2009; Li et al., 2009).

Both algorithms benefited somewhat from artifact blanking, more so for monkey M. For monkey $\mathrm{M}$, maximum accuracy was achieved with $5 \mathrm{~ms}$ of artifact blanking for both the WF and the UKF. For monkey N, maximum accuracy was achieved with 2 ms of artifact blanking for both decoders. These values reflect the difference in artifact duration and amplitude in two monkeys. The artifacts were more prominent and of longer duration in monkey $\mathrm{M}$ because of the close proximity of the stimulation site (hand representation of $\mathrm{S}_{1}$ ) to the area where neuronal activity was collected (arm representation of M1 and $\mathrm{S}_{1}$ ). The 


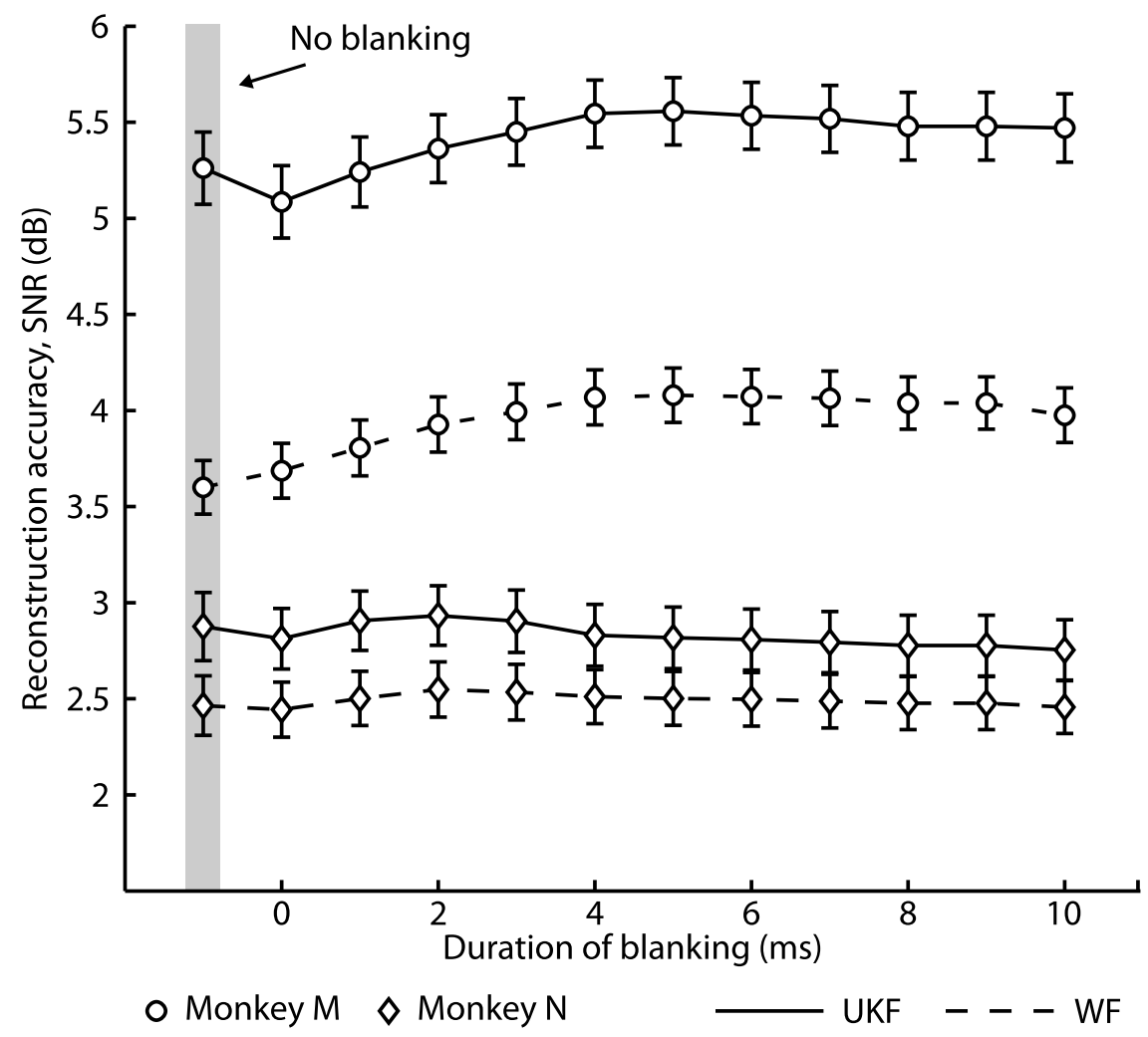

FIGURE 5.8: Exploration of blanking intervals. Mean movement reconstruction accuracy as a function of blanking intervals for both monkeys (symbols as in Figure 5.5) and both algorithms (Wiener filter, WF, dashed lines; unscented Kalman filter, UKF, solid lines). Bars indicate standard error. Shaded region corresponds to no blanking.

artifacts were smaller and of shorter duration for monkey $\mathrm{N}$, which received stimulation in the leg representation are of $S_{1}$ with recordings performed in the arm representation area.

Curiously, the performance of the UKF was slightly better for the no blanking condition than for $1 \mathrm{~ms}$ of blanking. This was because the recording channels that detected ICMS artifacts occasionally recorded additional mechanical artifacts related to monkey head movements. Apparently, the filter could use these mechanical artifacts that influenced the spike recording channels (blanked them or introduced erroneous spikes) to slightly improve predictions, and its performance was very slightly reduced when these artifacts 
were removed. This underscores the importance of registering the artifacts and removing them to minimize their influence on the filter performance.

To quantify the decrease in predictions caused by the presence of ICMS artifacts, we recorded from both monkeys as they performed a center-out task without any ICMS. For this task, they had to move the avatar from the center of the screen to a single peripheral object and hold for $2 \mathrm{~s}$. For monkey M, accuracy in this task was $23 \%$ higher than during the ICMS sessions with the UKF and 32\% higher with the WF. For monkey N, these values were $2.7 \%$ and $27 \%$, respectively. Thus, the artifacts worsened the predictions, even after blanking, but still within a tolerable range.

\subsection{Discussion}

This study continued our work on the development of an artificial somatosensory channel for BMIs (O’Doherty et al., 2009; Fitzsimmons et al., 2007, see also Chapter 3 and Chapter 4). Monkeys scanned virtual objects with an avatar hand and discriminated their artificial textures as represented by temporal patterns of ICMS. This paradigm meets the requirements of a clinically relevant neuroprosthetic arm sensorized with an artificial tactile channel. Such a neuroprosthetic arm could be used to touch external objects and estimate their tactile properties-roughness and smoothness, hardness and softness, wetness and dryness, and temperature-using sensors on the prosthetic hand. The transmission of this information to the nervous system is a difficult problem because of the artificial nature of the stimulation methods. We explored the capability of temporally patterned ICMS as a way to deliver somatosensory feedback to the brain by parametrically varying the degree of randomness of ICMS trains. Monkeys learned to distinguish regular ICMS patterns from irregular ones, which suggests that they were able to discriminate fine temporal structure of ICMS trains. Irregular bursts of sensory discharges are expected to occur in any practical neuroprosthesis, when the actuator interacts with realistically textured objects. 


\subsubsection{Prosthetic somatosensation}

Our results complement previous work on ICMS frequency discrimination conducted by Romo and colleagues (Romo et al., 1998, 2000; de Lafuente \& Romo, 2005) who trained their monkeys to discriminate periodic ICMS pulse trains. This study expanded the range of temporal patterns that could be represented by ICMS of $S_{1}$ by changing the regularity of the secondary frequency. Moreover, ICMS in our experiments served as somatosensory feedback during active touch, rather than acting merely as a cue in a forced choice task, as in the majority of previous studies. The animals actively explored virtual objects with an avatar hand, spending appropriate times over these objects as would be needed for normal interaction with the environment. Additionally, ICMS was delivered through chronically implanted electrodes, which allowed us to monitor long-term learning to utilize ICMS as sensory feedback. With studies conducted by other groups, stimulating electrodes were often inserted in the brain anew during each daily session. Long-term usage of ICMS in the present experiments (as well as previous experiments with the same monkeys) did not result in deterioration in performance, which indicates that the charge-balanced stimulus waveforms used here likely did not damage brain tissue.

These results showed that monkeys started to detect distortions in the $10 \mathrm{~Hz}$ ICMS secondary frequency after random variations of that frequency exceeded $25 \%$, that is, when the instantaneous frequency dropped below 7.5 or exceeded $12.5 \mathrm{~Hz}$. This estimate of the detection threshold can be used in future neuroprosthetic designs as a characteristic sensitivity value. Future studies should probe the sensitivity of discrimination to different primary and secondary frequencies. Additionally, spatiotemporal ICMS (Fitzsimmons et al., 2007) and ICMS of different duration should be explored as ways to encode information in BMBI sensory channels. 


\subsubsection{Artifact mitigation}

BMBIs with an afferent ICMS feedback loop need to compensate for electrical artifacts produced by ICMS pulses that may interfere with neuronal recordings. In our previous BMBI designs, we either discounted the entire period of ICMS application (O'Doherty et al., 2009, see also Chapter 3) or used interleaved recording and ICMS delivery intervals (see Chapter 4). These previous approaches limited the flexibility of ICMS delivery. In this study, we did not impose limitations on the timing of ICMS delivery and treated ICMS artifacts as they occurred. We found that blanking the periods after ICMS artifacts by short intervals ( 2 to $5 \mathrm{~ms}$ ) improved the accuracy of extraction of limb kinematics from neuronal activity. Overall accuracy of predictions were 20 to $30 \%$ less as compared to sessions in which ICMS was not used. Nonetheless, the predictions were still acceptable and in range of previously reported accuracy of BMI decoding. This result suggests that artifact blanking is practical for bidirectional neuroprostheses using irregular ICMS pulse trains.

The problem of stimulation artifacts will be compounded as multiple stimulation channels start to be employed with asynchronously delivered pulses. It is imperative that the masking of neuronal recordings, either indirectly by blanking intervals or other compensation strategies, or directly by the artifacts themselves, are minimized in the design of such systems. In the future, the problem of artifacts (Rolston et al., 2009), as well as the unreliable spatial extent of ICMS (Histed et al., 2009) could be mitigated by optogenetic stimulation (Boyden et al., 2005; Zhang et al., 2006, 2007; Huber et al., 2008).

\subsubsection{Qualia}

The question of the nature of the qualia of perceptions evoked by irregular temporal patterns of ICMS will have to wait until the technology is implemented in human subjects. However, our current and previous (O’Doherty et al., 2009; Fitzsimmons et al., 2007, Chapter 4) results suggest that new perceptions may evolve as subjects practice with ICMS. We always observed that it took monkeys 1 to 2 weeks to start to understand ICMS, even if they were previously 
overtrained with a vibrotactile variant in the same task. However, once they learned their first ICMS task, leaning subsequent tasks took much less time. The use of ICMS in active touch settings where subjects evoke ICMS and its associated sensations through their own actions (Sinclair \& Burton, 1988; Bolanowski et al., 2004; Simões-Franklin et al., 2010) may contribute to shaping the artificial perception and lead to the development of anticipatory cortical modulations similar to corollary discharge (Crapse \& Sommer, 2008).

The irregular ICMS patterns implemented in this study may convey other advantages as compared to strictly periodic ICMS pulse trains. For example, irregular ICMS may enhance sensory processing through the mechanism of stochastic resonance (McDonnell \& Abbott, 2009; Ward et al., 2010). This intriguing possibility should be experimentally tested. 


\section{6}

\section{Discussion}

He studies described in this dissertation were devoted to developing a new means
of delivering prosthetic somatosensation and incorporating this artificial modality into a BMI. This is significant because clinical BMIs must be bidirectional if they are to be clinically useful. A prosthetic arm, even one controlled by the brain, might not convey a sense of agency or ownership to the user. Additionally, somatosensory feedback would enable the users of a neuroprosthesis to engage in natural behaviors that they would not be able to otherwise accomplish. For example, prosthetic somatosensation would enable interaction with the environment without (or with reduced) visual attention. This would enable users to select coins in a pocket, or reach for objects outside of their visual field.

Below, I discuss the specific contributions of this dissertation, with special emphasis on their relevance to engineering. I next discuss a few of the unresolved questions which arise from the work accomplished here. Finally, I propose several extensions and future avenues of research which subsequent investigators may find fruitful. 


\subsection{Engineering Contributions}

\subsubsection{Sensory input concurrent with BMI}

First, a means of combining an efferent brain-machine interface with an afferent somatosensory channel was engineered. Under the proposed scheme, somatosensory input to the brain would be mediated by patterns of electrical stimulation of cortical neurons. In Chapter 3 I described the development and validation of a such a system in an animal model. This demonstration required the development of brain-machine interface software and algorithms and electrical stimulation hardware and protocols and was the first demonstration of a brain-machine interface with an artificial sensory channel delivered directly to the brain.

\subsubsection{Interleaved stimulation and recordings}

One of the principal challenges immediately apparent at the outset of this work was in achieving reliable electrical recordings simultaneous with electrical stimulation of the brain. This challenge was overcome through the development of an interleaved stimulation and recording paradigm, as described in Chapter 4. By alternating between stimulation and recording intervals in rapid succession, the monkey was endowed with effectively simultaneous action and perception through the BMBI. This practical solution allowed the monkey to interact with the environment with a latency short enough to enable artificial active touch. This was the first demonstration of a BMI that enabled active touch.

\subsubsection{Controlled aperiodic patterns of stimulation}

Finally, I engineered a method of probing the sensitivity of somatosensory cortex to precisely controlled temporal patterns of electrical stimulation. By presenting periodic and aperiodic patterns of ICMS (see Chapter 5) with matched mean pulse rates and titrated levels of variability I quantitatively measured an upper bounds on the bandwidth that can be directly delivered to somatosensory cortex using patterns of ICMS. Moreover, I measured the consequences of using different lengths of artifact blanking on the quality of neural record- 
ings. These findings provide a basis for selecting patterns of ICMS for more sophisticated spatiotemporal protocols.

\subsection{Unresolved questions}

\subsubsection{The question of qualia}

It is not known how the animal subjects in these experiments perceived the patterns of ICMS that they received. This question unfortunately cannot be directly answered in this, or any, animal model. However, there are several lines of evidence that are very suggestive. First, electrodes with neuronal receptive fields localized to specific peripheral body sites were selected for ICMS. It is therefore possible that similar networks of neurons were activated by both ICMS and touch. Second, neuroimaging studies have demonstrated the correspondence between stimulated sites on the body, brain map activation, and report of localizations (Burton et al., 1993). Third, ICMS of somatosensory cortex in humans evokes sensations that are ascribed to the body site whose map is at the focus of stimulation (Penfield \& Boldrey, 1937). Fourth, patients with limb amputations often experience sensations that are localized to a phantom limb. In some of these cases, neuroimaging shows activation of the brain map region that would have been activated when the intact limb was touched (Ramachandran, 1993). Therefore, by stimulating an animal in a particular $S_{1}$ map region, it is reasonable to presume that they experience a perception ascribed to that part of the body.

\subsubsection{The timescales of learning of S1 ICMS patterns}

In the experiments described here, and in our previous studies (Fitzsimmons et al., 2007; O'Doherty et al., 2009), it took monkeys several sessions to learn to interpret ICMS patterns, but once they started to respond they were able to master tasks with increasing complexity and to maintain proficient performance for many months. For the experiments described in Chapter 3 and Chapter 4 , the monkeys were trained on a vibrotactile version of the task. However, in both cases, as well as in a previous study (Fitzsimmons et al., 2007), we did not 
observe an immediate transfer of ability when the monkeys switched from the vibrotactile to the ICMS version of the task. Rather it took several sessions for them to begin to use ICMS at greater than chance levels. This could indicate a substantial difference between the sensations evoked by skin vibration and ICMS. Alternatively, it could mean that evoked perceptions did not occur immediately, but rather took time and exposure to develop.

These results are different from those of Romo and colleagues (Romo et al., 1998), who reported almost immediate transfer from a vibrotactile to an ICMS task. One explanation for this discrepancy is that the specific $S_{1}$ subdivision studied by Romo and colleagues, area $3 \mathrm{~b}$, produces more natural sensations than stimulation of area 1 , the area implanted in the experiments reported here, Additionally, Romo and colleagues conducted a number of initial test sessions during which the monkeys could have learned to discriminate ICMS.

\subsubsection{Long-term effects of ICMS}

Despite receiving trains of ICMS for up to an hour a day a week, five days a week, for in excess of a year at the time of writing, there was no evidence of degradation in the monkeys' ability to successfully use ICMS. Nor did we observe any negative effects on their health or well-being. This suggests that patterns of ICMS may be safely and effectively delivered through chronically implanted multi-electrode arrays over long intervals. While much more extensive studies will be necessary before such technology may be transferred to human patients, these studies provide initial evidence for the viability of the approach.

\subsubsection{Prosthesis incorporation into the body schema}

One important requirement for any practical neuroprostheses is that it acts and feels as the subject's natural limbs (Lebedev \& Nicolelis, 2006). Here, this goal was approached by incorporating in the BMBI an avatar arm that was controlled by cortical activity and evoked ICMS patterns when virtual objects were touched. Cortical ensembles were found to be modulated even if the avatar arm was moved for the monkey rather than by the monkey. This finding 
was similar to that of Graziano and colleagues who found that posterior parietal cortex neurons responded to the positioning of a taxidermically prepared monkey arm (Graziano et al., 2000). We suggest that concurrent sensory and motor loops could be used to gradually incorporate a neuroprosthetic limb into a human subject's internal representation. Taking this idea even further, it is reasonable to suggest that future applications of BMBIs will not be limited to limb prostheses, but may include a range of devices designed for reciprocal communication between neural structures and a variety of external devices or other neural structures. In these applications, ICMS may be used to replicate normal sensations or even represent new physical parameters.

\subsubsection{Requirement of somatotopy}

While the monkeys in this study were used as models of amputee or spinal cord injured patients, they had intact limbs and cortical structures with normal connectivity and somatotopy. However, a candidate recipient of a clinical neuroprosthetic limb would possess a sensorimotor cortex exhibiting profound plastic changes (Pons et al., 1991) due to denervation as a result of limb loss or spinal cord injury. It is unknown whether the BMBI developed here would be successful in such a situation. However, it has been reported that the sensory cortex recovers a somewhat typical organization after allogeneic hand transplantation (Frey et al., 2008), presumably due to renewed sensory input. It is possible that patterns of ICMS delivered to $\mathrm{S}$, congruent with active touch using a neuroprosthesis, could retrain the lost cortical region, possibly even resulting in a recovery of the cortical map.

\subsubsection{Sensory substitution}

Sensory substitution (Kaczmarek et al., 1991) is an alternative to using ICMS for sensorizing neuroprostheses. This is the use of an intact sensory modality as a "stand-in" for an unavailable sensory modality. The user is then able to learn to interpret the substituted sensory channel as if it were the lost modality, and through a process of cortical plasticity 
may even begin to perceive it was the lost modality (Bach-y-Rita \& Kercel, 2003). A variety of vibrotactile (Cincotti et al., 2007) and electrotactile (Scott et al., 1980) strategies have been developed.

Sensory substitution has the clear advantage over ICMS of being much less invasive. However, sensory substitution has several disadvantages of its own. First, each independent channel of sensory substitution requires an actuator to be placed on the skin. In order to maximize the bandwidth, this is often placed on an area with high mechanoreceptor density (e.g. the tongue). It may be necessary to remove the stimulator when other activities are to occur (e.g. speaking). More convenient stimulator locations, such as the skin of the back, may require much larger stimulators and could lack the necessary bandwidth for practical applications. In the case of ICMS, it is easy to envision that a much higher density of stimulating electrodes could be achieved than with peripheral stimulators. Furthermore, stimulation could be targeted at the part of the sensory map corresponding to the intended sensation rather than at a substitute location. Finally, sensory substitution is almost completely unavailable for persons with spinal cord injuries, for whom large portions of their bodies are numb. Indeed, in this patient population, ICMS may be the only viable strategy for restoring somatic sensation.

\subsubsection{Can any sensory cortical region be used as a target for ICMS?}

It is possible that any postcentral cortical region could serve as an appropriate site for feedback about the somatosensory properties of a neuroprosthesis. Indeed, the fact that sensory substitution is so effective speaks to the remarkable plasticity of the cortex. It is true that in this study PPC was found to be ineffective in one monkey. However, as a negative result, this finding is challenging to interpret. First, this finding was only shown in one monkey. Second, it is possible that the monkey could have learned to use ICMS of PPC if more time was given. Third, it is possible that the electrodes penetrated cortex at a depth too deep or too shallow (or had some other localization malfunction) for recruitment of 
the appropriate neurons. Future studies should be conducted to confirm or disprove this finding.

\subsection{Future Work}

\subsubsection{Improved artifact mitigation}

In Chapter 5, I introduced a paradigm in which each ICMS pulse-or short train of pulsescan have significant temporal variability. Such a scheme would render infeasible the simple interleaved stimulation and recording intervals described in Chapter 4 because the amount of blanking required could vary considerably with time. This problem would be further compounded with the introduction of spatiotemporal ICMS, where at nearly any given moment there could be pulse trains being delivered on one or more channels (see Subsection 6.3.4). As a result, improved methods for artifact mitigation will need to be considered. One possibility would be to switch the recording amplifiers out of the circuit when ICMS pulses are delivered to prevent the recording amplifiers from being saturated (Freeman, 1971; Jimbo et al., 2003; Venkatraman et al., 2009). This alone may reduce the effective duration of the artifact less than $1 \mathrm{~ms}$ and would allow stimulation and recording on the same channel. Such switches may be too large and bulky to be practical, especially for clinical settings. Other options include specialized recording amplifiers (Knaflitz \& Merletti, 1988; Rossi et al., 2007; Rolston et al., 2009) and software subtraction of the artifact (Wagenaar \& Potter, 2002). It remains to be seen which artifact mitigation method will convey the most benefits.

Another way to reduce the duration ICMS artifacts is to use shorter pulse trains. Recent work from Butovas \& Schwarz (2007) demonstrated that double pulses achieve an effect nearly as large as what is seen when much longer pulse trains are used. This suggests that such doublets could be effectively used to drastically reduce the times which stimulation is delivered with minimal affect on stimulus salience. 


\subsubsection{Optogenetic ICMS for prosthetic somatosensation}

In the future, other methods for the stimulation of neural tissue may complement ICMS. One promising method is optogenetic ICMS (Deisseroth, 2011). While this technique requires a transgenic animal or the use of viral vectors to deliver genes coding for light-sensitive proteins, it confers several advantages over electrical ICMS. First, optogenetic stimulation can be used to both excite (Boyden et al., 2005) or inhibit (Zhang et al., 2007) neural structures using different frequencies of light. Second, depending on the promoter, optogenetic stimulation can activate or inhibit specific cell types, for example just excitatory or just inhibitory neurons. This is in contrast to electrical ICMS which recruits sparse unpredictable populations of neurons without any cell-type specificity (Histed et al., 2009), and is believed to recruit axons with lower thresholds than cell bodies (Ranck, 1975). Third, optogenetic stimulation produces smaller artifacts in electrical recording channels than electrical ICMS (Cardin et al., 2010). Still, chronic electrical stimulation of the nervous system has a long track record of safety and efficacy in animals and humans, unlike optogenetics, which may pose as of yet unknown risks. Gilja et al. (2011) propose some of the current ideas in development for optogentic ICMS for prosthetic somatosensation.

\subsubsection{Representing texture with gratings}

In the experiments described in Chapter 4 and Chapter 5 , the delivery of a pattern of ICMS corresponding with a particular AT was triggered by the avatar crossing the edge of the virtual object. This allowed surface-hand interactions to be modeled in a simpler way. However, actual interactions of glabrous skin with surfaces is considerably more complicated, with compressive strain of the skin surface necessary for mechanoreceptor activation and tactile perception (Van Doren, 1989; Srinivasan \& Dandekar, 1996). Therefore, future studies should employ a more realistic model of object-hand interactions and ICMS delivery.

One promising design for the next generation of somatosensory neuroprostheses would be to deliver a short pulse train of ICMS whenever the avatar hand came into contact with 

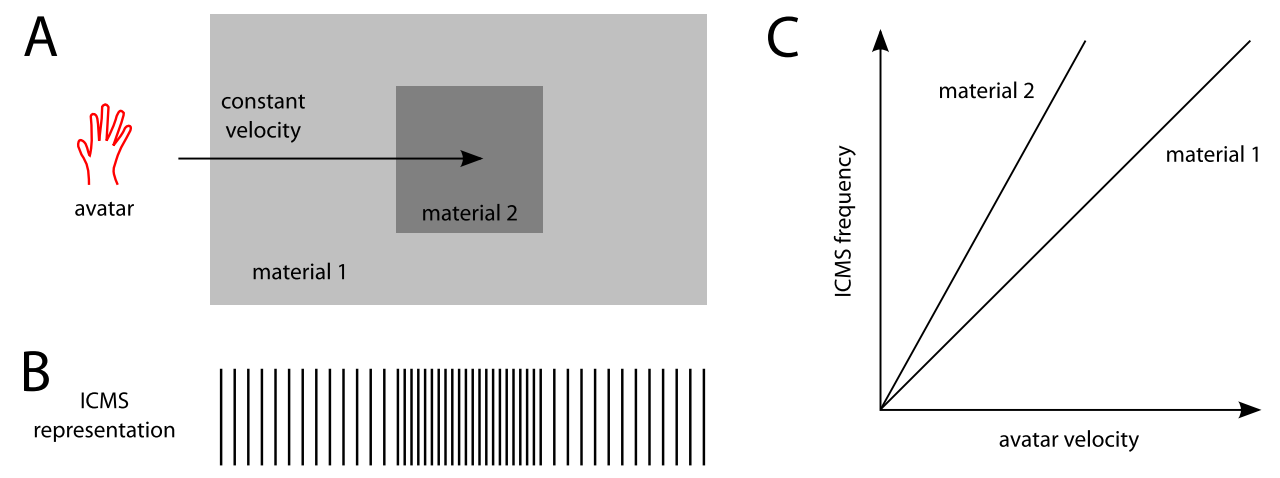

FIGURE 6.1: Representing tactile gratings with ICMS patterns. A. Schematic of the task paradigm. Two or more virtual objects are presented on the screen as surfaces consisting of tactile gratings with different spatial frequencies and orientations. For simplicity the gratings in this example are both perpendicular with respect to the movement of the avatar, which is moving with a constant velocity. B. Representation of a possible ICMS pulse train delivered as the avatar moves across the virtual materials depicted in A. C. Diagram of how the frequency of ICMS delivered depends on velocity for each of the materials depicted in A.

a relevant texture feature on a surface. For a clinical neuroprosthesis, the texture features (microscopic ridges and valleys on the surface of objects) could be measured by sensors embedded in the prosthetic hand. For initial animal experiments, the texture features could be represented with tactile gratings and plaids (Pei et al., 2011). Each time that the avatar hand (or other actuator) touches the ridge on a grating, a brief of ICMS would be delivered to a cortical channel in S1. Of course, the ridges would not be visible, as with visual gratings, but could only be comprehended through prosthetic touch. As the avatar hand moved across the surface of the object the precise temporal pattern of ICMS would depend on the velocity of the avatar hand with respect to the parameters of the virtual tactile gratings (spatial frequency and angle). See Figure 6.1 for a cartoon of this idea.

There are a number of issues that would need to be resolved before such a scheme could be implemented. Chief among them is that the perceived roughness has been found to be invariant of the speed at which textured surfaces move underneath the fingertips (Lederman, 
1974), suggesting that temporal codes are not by the brain to represent roughness. Alternatively, it could be that temporal codes are used but that corollary discharge (Crapse \& Sommer, 2008) from motor cortical areas to sensory cortical areas compensates for the velocity dependent activity of peripheral mechanoreceptors. Whether the simple ICMS scheme described here is sufficient to evoke naturalistic sensations of texture remains to be seen.

\subsubsection{Spatiotempral ICMS}

In the present study, frequency patterning was used to represent the different artificial textures. In the quest to attain more naturalistic representations of texture, particularly modalities beyond the roughness/smoothness axis, it may be desirable to vary other parameters of the ICMS pulse trains. One candidate would be to deliver spatiotemporally patterned ICMS over a large number of the electrodes on the array. If the underlying cortical tissue contains somatotopic maps, this type of stimulation could be used to mimic the perception of the motion of objects against the skin, or allow for the users of a neuroprosthetic arm to manipulate 3-D objects and perceive their form. Ideally, the use of spatiotemporal ICMS would allow the users of a neuroprosthesis to employ a variety of natural "exploratory procedures", such as lateral motion, enclosure, and contour following to gain knowledge about the properties of objects in the environment (Lederman \& Klatzky, 1987).

While there is evidence that the primate brain can learn to use fairly arbitrary spatiotemporal patterns of ICMS (Fitzsimmons et al., 2007), for a clinical neuroprosthesis, it will probably be preferable to take advantage of existing somatotopy. For example, electrodepairs implanted in the different digit and palm areas of the hand would correspond to tactile sensors embedded in the homologous areas of the robotic hand or artificial actuator. As the hand is used to touch and manipulate objects, patterns of ICMS would be delivered to the appropriate cortical channels, resulting in a spatiotemporal wave of activity across somatosensory cortex. As it has been hypothesized that the quality of perceived roughness 
is proportional to the amount of skin deformation caused by the ridges of a grooved surface (Lederman, 1974), the use of the spatiotemporal encoding scheme proposed here would probably require a fairly dense grid of stimulating electrodes. See Figure 6.2 on the following page for a cartoon of this idea.

\subsubsection{Closed-loop control of ICMS parameters}

Evoked cortical activity exhibits significant variability for repeated presentations of the same peripheral sensory (Arieli et al., 1996) and central electrical stimuli (Schmidt et al., 1996; Butovas \& Schwarz, 2003), presumably due to ongoing neuronal activity (Petersen et al., 2003). This is of consequence for prosthetic applications of ICMS because the quality of an ICMS evoked perception, and indeed whether the subject perceives anything at all, may depend on neural processing related to these evoked potentials. To address this challenge, Brugger and colleagues recently proposed the application of closed-loop control of stimulation amplitude (Brugger et al., 2011). By sensing the state of cortical networks by measuring local field potential activity, the authors were able to predict the activity that would be evoked by varying ICMS pulses with amplitudes, and were able to modulate the amplitude of each pulse to reduce the variability of the resultant evoked cortical activity. It is not yet known if this reduction on variability leads to a corresponding stabilization of perceived responses, but this possibility should be checked in future studies on prosthetic somatosensation.

\subsubsection{Proprioception and pain}

In this study we incorporated an artificial tactile channel in a BMI-ICMS provided information about whether objects were associated with reward or not, and monkeys could probe their object identity in the same was as if they felt them with their hands. However, there are other somatosensory modalities that may also be good candidates for incorporation into future clinical neuroprosthetic devices. I briefly discuss two others here: proprioception and pain. 
A

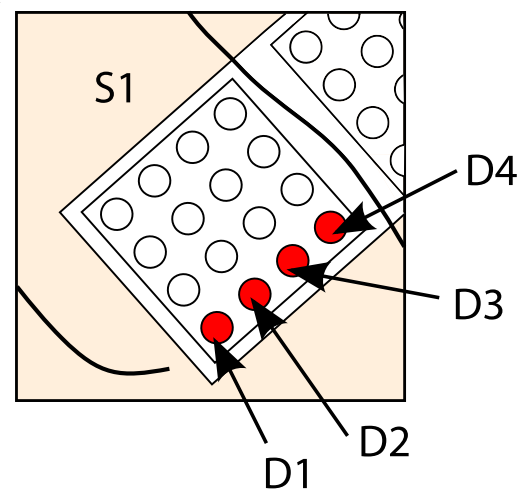

B

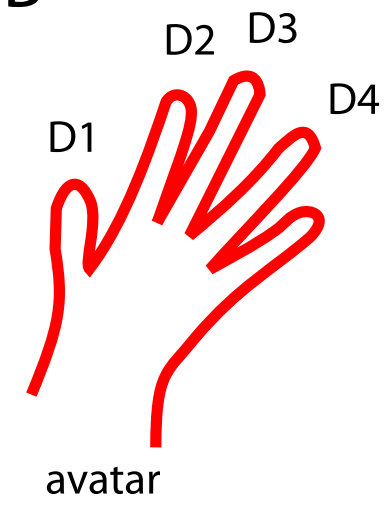

C

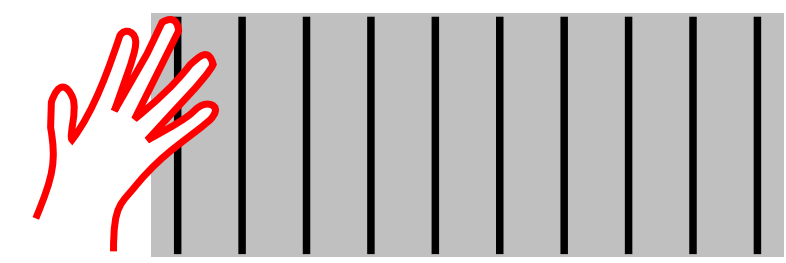

constant velocity

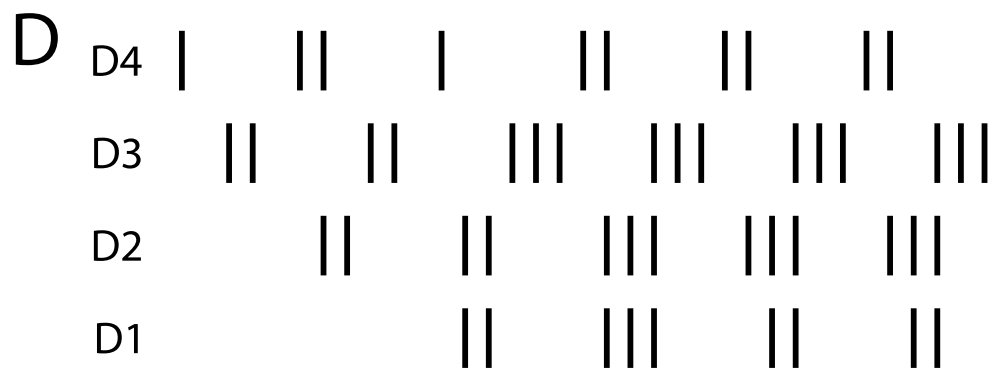

FIGURE 6.2: Spatiotemporal patterns of ICMS for representing texture. A. Diagram indicating four electrodes in the hand representation area of S1. Labels indicate the digits of the avatar, which will be represented on each channel. B. Four homologous digits on a cartoon of the avatar hand that correspond with the channels in A. C. As the avatar moves over a textured surface, shown here as a tactile grating, a pulse or short pulse train of ICMS is delivered as each digit of the avatar moves over each of the ridges of the grating. Motion here is shown as constant velocity and in a straight line perpendicular to the grating. D. Cartoon representing a possible spatiotemporal pattern of ICMS that would be evoked as the avatar hand moves across the grating shown in C. 
Proprioception is the awareness of the position and orientation of the various parts of the body. A prosthetic proprioceptive sense would be of obvious utility for the users of a neuroprosthesis because it would enable them to safely and comfortably use their robotic appendages without directly visually attending to them. The neuronal bases of proprioception and kinesthesia are not fully understood, and are likely to involve numerous brain regions, but areas 3 a and 2 stand out as strong candidate targets for ICMS. London and colleagues have demonstrated that monkeys can discriminate between different frequencies of ICMS applied to area 3a, but to date ICMS of area za has not successfully been used to provide a useful proprioceptive signal. This possibility should be pursued further.

A prosthetic sense of pain is likewise problematic. Stimulation of the cortex has rarely produced a sensation of pain in humans (Penfield \& Boldrey, 1937), nor is pain perception a typical consequence of cerebral damage. There is some evidence that the thalamus and secondary somatosensory cortex are involved in pain perception (Craig et al., 1994), but it is more likely that pain is represented in a distributed fashion across many cortical areas.

Some analog of pain would of course be useful so that the users of a neuroprosthesis would protect it from damage. But is it necessary for it to feel like "pain"? It may not be truly desirable to provide the users of a neuroprosthesis with an experience of the noxious aspects of pain. A more humane approach would be provide a very salient prosthetic tactile experience that can act as a stand-in for noxious pain, providing the advantages without the negative affect.

\subsubsection{Incorporation of tactile sensors into physical devices}

In the studies described here, monkeys interacted with virtual objects using an upper limb avatar. This simplified the experimental apparatus required, but was also significantly less realistic than a clinical neuroprosthesis, which would necessarily involve the interaction of a robotic limb-or perhaps an electrically stimulated limb-with physical objects in a real-world environment. This would require that the robotic appendage be sensorized with 
with an array of tactile sensors capable of detecting the tactile qualities of physical objects. If the user's arm is animated via functional electrical stimulation, the tactile sensors would instead be embedded in a glove or sleeve. The recorded tactile information would then be transmitted to the cortical implant, transformed into a spatiotemporal pattern, and delivered through ICMS.

\subsubsection{Avatar incorporation experiments in humans}

In Chapter 4, I provided evidence that the monkey avatar arm became incorporated into monkey's internal brain representation in the form of directional tuning to the movements of the passively moved avatar arm (see Figure 4.16 on page 80 ). The underlying assumption here is that when a limb feels like it belongs to an individual, there is a corresponding change in neuronal activity reflecting this perception. While the exact nature and extent of these hypothesized changes are not known, there is a suggestion from the human rubber hand illusion literature that activity in premotor cortex (Ehrsson et al., 2004), primary (Schaefer et al., 2006, 2007) and secondary (Keysers et al., 2004) somatosensory cortex, and other parietal regions (Maravita et al., 2003) are involved in the construction of the body schema and peripersonal space. However, in all these cases the illusion of body ownership is transient, probably because the subject is unable to volitionally move the rubber hand. This is not the case for a brain-controlled neuroprosthesis.

Notwithstanding a recent report investigating the effect of virtual environments on the sensation of presence for users of brain-computer interfaces (Groenegress et al., 2010) there has not been a systematic exploration of the factors that contribute to the users of a neuroprosthesis feeling that their prosthetic limb belongs to them, nor how the addition of somatic sensation contributes to those perceptions, nor what the neuronal activity underlying those perceptions might be. This avenue warrants much further study. 


\section{Bibliography}

Ahuja, A. K., Dorn, J. D., Caspi, A., McMahon, M. J., Dagnelie, G., Dacruz, L., Stanga, P., Humayun, M. S., Greenberg, R. J., \& Group, A. I. S. (2011). Blind subjects implanted with the argus ii retinal prosthesis are able to improve performance in a spatial-motor task. $\mathrm{Br}$ J Ophthalmol, 95, 539-543. (p. 11)

Arieli, A., Sterkin, A., Grinvald, A., \& Aertsen, A. (1996). Dynamics of ongoing activity: explanation of the large variability in evoked cortical responses. Science, 273, 1868-1871. (p. 113)

Atkins, D., Heard, D., \& Donovan, W. (1996). Epidemiologic overview of individuals with upper-limb loss and their reported research priorities. Journal of Prosthetics and Orthotics, 8, 2-11. (p. 59)

Bach-y-Rita, P., Collins, C. C., Saunders, F. A., White, B., \& Scadden, L. (1969). Vision substitution by tactile image projection. Nature, 221, 963-964. (p. 85)

Bach-y-Rita, P., Kaczmarek, K. A., Tyler, M. E., \& Garcia-Lara, J. (1998). Form perception with a 49-point electrotactile stimulus array on the tongue: a technical note. J Rehabil Res Dev, 35, 427-430. (p. 85)

Bach-y-Rita, P. \& Kercel, S. W. (2003). Sensory substitution and the human-machine interface. Trends Cogn Sci, 7, 541-546. (p. 85, 108)

Basmajian, J. V. (1963). Control and training of individual motor units. Science, 141, 440-441. (p. 3)

Berger, T. W., Ahuja, A., Courellis, S. H., Deadwyler, S. A., Erinjippurath, G., Gerhardt, G. A., Gholmieh, G., Granacki, J. J., Hampson, R., Hsaio, M. C., LaCoss, J., Marmarelis, V. Z., Nasiatka, P., Srinivasan, V., Song, D., Tanguay, A. R., \& Wills, J. (2005). Restoring lost cognitive function. IEEE Eng Med Biol Mag, 24, 30-44. (p. 12)

Birbaumer, N. (2006). Brain-computer-interface research: coming of age. Clin Neurophysiol, $117,479-483$. (p. 3,8$)$

Bolanowski, S. J., Verrillo, R. T., \& McGlone, F. (2004). Passive, active and intra-active (self) touch. Behav Brain Res, 148, 41-45. (p. 102) 
Boyden, E. S., Zhang, F., Bamberg, E., Nagel, G., \& Deisseroth, K. (2005). Millisecondtimescale, genetically targeted optical control of neural activity. Nat Neurosci, 8, 1263-1268. (p. 82, 101, 110)

Brindley, G. S. \& Lewin, W. S. (1968). The sensations produced by electrical stimulation of the visual cortex. J Physiol (Lond), 196, 479-493. (p. 11)

Brown, T. G. \& Sherrington, C. S. (1911). Observations on the localisation in the motor cortex of the baboon ("papio anubis"). J Physiol (Lond), 43, 209-218. (p. 10)

Brugger, D., Butovas, S., Bogdan, M., \& Schwarz, C. (2011). Real-time adaptive microstimulation increases reliability of electrically evoked cortical potentials. IEEE Transactions on Biomedical Engineering. (p. 113)

Brumberg, J. S., Nieto-Castanon, A., Kennedy, P. R., \& Guenther, F. H. (2010). Braincomputer interfaces for speech communication. Speech Commun, 52, 367-379. (p. 85)

Burton, H., Videen, T. O., \& Raichle, M. E. (1993). Tactile-vibration-activated foci in insular and parietal-opercular cortex studied with positron emission tomography: mapping the second somatosensory area in humans. Somatosens Mot Res, 10, 297-308. (p. 105)

Butovas, S. \& Schwarz, C. (2003). Spatiotemporal effects of microstimulation in rat neocortex: a parametric study using multielectrode recordings. J Neurophysiol, 90, 3024-3039. (p. 113)

Butovas, S. \& Schwarz, C. (2007). Detection psychophysics of intracortical microstimulation in rat primary somatosensory cortex. Eur J Neurosci, 25, 2161-2169. (p. 57, 85, 109)

Cardin, J. A., Carlén, M., Meletis, K., Knoblich, U., Zhang, F., Deisseroth, K., Tsai, L.-H., \& Moore, C. I. (2010). Targeted optogenetic stimulation and recording of neurons in vivo using cell-type-specific expression of channelrhodopsin-2. Nature Protocols, 5, 247-254. (p. 110)

Carmena, J. M., Lebedev, M. A., Crist, R. E., O’Doherty, J. E., Santucci, D. M., Dimitrov, D. F., Patil, P. G., Henriquez, C. S., \& Nicolelis, M. A. L. (2003). Learning to control a brain-machine interface for reaching and grasping by primates. PLoS Biol., 1, E42. (p. 6, $23,31,39,57,59,66,73,75,78,87,93)$

Chapin, J. K., Moxon, K. A., Markowitz, R. S., \& Nicolelis, M. A. L. (1999). Real-time control of a robot arm using simultaneously recorded neurons in the motor cortex. Nat Neurosci, 2, 664-67o. (p. 6, 59)

Chatterjee, A., Aggarwal, V., Ramos, A., Acharya, S., \& Thakor, N. V. (2007). A braincomputer interface with vibrotactile biofeedback for haptic information. J Neuroengineering Rehabil, 4, 40. (p. 56) 
Cheng, G., Fitzsimmons, N. A., Morimoto, J., Lebedev, M. A., Kawato, M., \& Nicolelis, M. A. L. (2007). Bipedal locomotion with a humanoid robot controlled by cortical ensemble activity. In Soc. Neurosci. Abstr., p. 517.22. (p. 7)

Chorover, S. L. \& DeLuca, A. M. (1972). A sweet new multiple electrode for chronic single unit recording in moving animals. Physiol Behav, 9, 671-674. (p. 5)

Cincotti, F., Kauhanen, L., Aloise, F., Palomäki, T., Caporusso, N., Jylänki, P., Mattia, D., Babiloni, F., Vanacker, G., Nuttin, M., Marciani, M. G., \& del R Millán, J. (2007). Vibrotactile feedback for brain-computer interface operation. Computational Intelligence and Neuroscience, p. 48937. (p. 56, 59, 108)

Cohen, E. D. (2007). Prosthetic interfaces with the visual system: biological issues. Journal of neural engineering, 4, R14-31. (p. 85)

Craig, A. D., Bushnell, M. C., Zhang, E. T., \& Blomqvist, A. (1994). A thalamic nucleus specific for pain and temperature sensation. Nature, 372, 770-773. (p. 115)

Crapse, T. B. \& Sommer, M. A. (2008). Corollary discharge circuits in the primate brain. Curr Opin Neurobiol, 18, 552-557. (p. 31, 102, 112)

Cushing, H. (1909). A note upon the faradic stimulation of the postcentral gyrus in conscious patients. Brain, 32, 44-53. (p. 10)

Dagnelie, G. (2008). Psychophysical evaluation for visual prosthesis. Annu Rev Biomed Eng, 10, 339-368. (p. 31, 85)

de Lafuente, V. \& Romo, R. (2005). Neuronal correlates of subjective sensory experience. Nat Neurosci, 8, 1698-1703. (p. 12, 85, 100)

Deisseroth, K. (2011). Optogenetics. Nat Meth, 8, 26-29.(p. 110)

Deliano, M., Scheich, H., \& Ohl, F. W. (2009). Auditory cortical activity after intracortical microstimulation and its role for sensory processing and learning. J Neurosci, 29, 1589815909. (p. 11)

Dobelle, W. H., Mladejovsky, M. G., Evans, J. R., Roberts, T. S., \& Girvin, J. P. (1976). "braille" reading by a blind volunteer by visual cortex stimulation. Nature, 259, 111-112. (p. 11)

Dobelle, W. H., Mladejovsky, M. G., \& Girvin, J. P. (1974). Artifical vision for the blind: electrical stimulation of visual cortex offers hope for a functional prosthesis. Science, 183, 440-444. (p. 11, 31, 85)

Dorval, A. D., Bettencourt, J., Netoff, T. I., \& White, J. A. (2007). Hybrid neuronal network studies under dynamic clamp. Methods Mol Biol, 403, 219-231. (p. 12) 
Dorval, A. D., Kuncel, A. M., Birdno, M. J., Turner, D. A., \& Grill, W. M. (2010). Deep brain stimulation alleviates parkinsonian bradykinesia by regularizing pallidal activity. $\mathrm{J}$ Neurophysiol, 104, 911-921. (p. 90)

Dushanova, J. \& Donoghue, J. P. (2010). Neurons in primary motor cortex engaged during action observation. Eur J Neurosci, 31, 386-398. (p. 8o)

Edgerton, B. J., House, W. F., \& Hitselberger, W. (1982). Hearing by cochlear nucleus stimulation in humans. Ann Otol Rhinol Laryngol Suppl, 91, 117-124. (p. 11)

Ehrsson, H. H., Spence, C., \& Passingham, R. E. (2004). That's my hand! activity in premotor cortex reflects feeling of ownership of a limb. Science, 305, 875-877. (p. 116)

Eisen, M. D. (2003). Djourno, eyries, and the first implanted electrical neural stimulator to restore hearing. Otol Neurotol, 24, 500-506. (p. 11)

Evarts, E. V. (1968). Relation of pyramidal tract activity to force exerted during voluntary movement. J Neurophysiol, 31, 14-27. (p. 4)

Fagg, A. H., Hatsopoulos, N. G., de Lafuente, V., Moxon, K. A., Nemati, S., Rebesco, J. M., Romo, R., Solla, S. A., Reimer, J., Tkach, D. C., Pohlmeyer, E. A., \& Miller, L. E. (2007). Biomimetic brain machine interfaces for the control of movement. J Neurosci, 27, 1184211846. (p. 12)

Fagg, A. H., Hatsopoulos, N. G., London, B. M., Reimer, J., Solla, S. A., Wang, D., \& Miller, L. E. (2009). Toward a biomimetic, bidirectional, brain machine interface. Conference proceedings : ... Annual International Conference of the IEEE Engineering in Medicine and Biology Society. IEEE Engineering in Medicine and Biology Society. Conference, 2009, 3376-3380. (p. 85)

Fallon, J. B., Irvine, D. R. F., \& Shepherd, R. K. (2008). Cochlear implants and brain plasticity. Hear Res, 238, 110-117. (p. 31, 85)

Farwell, L. A. \& Donchin, E. (1988). Talking off the top of your head: toward a mental prosthesis utilizing event-related brain potentials. Electroencephalogr Clin Neurophysiol, 70, 510-523. (p. 8)

Fetz, E. E. (1969). Operant conditioning of cortical unit activity. Science, 163, 955-958. (p. 3)

Fetz, E. E. (2007). Volitional control of neural activity: implications for brain-computer interfaces. J Physiol (Lond), 579, 571-579. (p. 31)

Fetz, E. E. \& Baker, M. A. (1973). Operantly conditioned patterns on precentral unit activity and correlated responses in adjacent cells and contralateral muscles. J Neurophysiol, 36, 179-204. (p. 3) 
Fetz, E. E. \& Finocchio, D. V. (1971). Operant conditioning of specific patterns of neural and muscular activity. Science, 174, 431-435. (p. 3)

Fetz, E. E. \& Finocchio, D. V. (1975). Correlations between activity of motor cortex cells and arm muscles during operantly conditioned response patterns. Exp Brain Res, 23, 217-240. (p.3)

Fetz, E. E. \& Wyler, A. R. (1973). Operantly conditioned firing patterns of epileptic neurons in the monkey motor cortex. Exp Neurol, 40, 586-607. (p.3)

Finkle, I. L. (1965). Recording lissajous figures. Science, 148, 1541-1542. (p. 36)

Fisher, R. A. (1936). The use of multiple measurements in taxonomic problems. Annals of Eugenics, 7, 179-188. (p. 38)

Fitzsimmons, N. A., Drake, W., Hanson, T. L., Lebedev, M. A., \& Nicolelis, M. A. L. (2007). Primate reaching cued by multichannel spatiotemporal cortical microstimulation. J Neurosci, 27, 5593-5602. (p. 12, 54, 55, 56, 57, 60, 85, 86, 89, 99, 100, 101, 105, 112)

Fitzsimmons, N. A., Lebedev, M. A., Peikon, I. D., \& Nicolelis, M. A. L. (2009). Extracting kinematic parameters for monkey bipedal walking from cortical neuronal ensemble activity. Front. Integr. Neurosci., 3, 3. (p. 7, 85, 97)

Flanagan, J. R. \& Wing, A. M. (1993). Modulation of grip force with load force during point-to-point arm movements. Exp Brain Res, 95, 131-143. (p. 59)

Foerster, O. (1936). The motor cortex in man in the light of hughlings jackson's doctrines. Brain, 59, 135. (p. 10, 11)

Fountas, K. N. \& Smith, J. R. (2007). A novel closed-loop stimulation system in the control of focal, medically refractory epilepsy. Acta Neurochir Suppl, 97, 357-362. (p. 13)

Frank, K. (1968). Some approaches to the technical problem of chronic excitation of peripheral nerve. Ann Otol Rhinol Laryngol, 77, 761-771. (p. viii, 4)

Franklin, B. (1757). An account of the effects of electricity in paralytic cases. in a letter to john pringle, m. d. f. r. s. from benjamin franklin, esq; f. r. s. Philosophical Transactions (1683-1775), 50, 481-483. (p. 9)

Freeman, J. A. (1971). An electronic stimulus artifact suppressor. Electroencephalography and clinical neurophysiology, 31, 170-172. (p. 109)

Frey, S. H., Bogdanov, S., Smith, J. C., Watrous, S., \& Breidenbach, W. C. (2008). Chronically deafferented sensory cortex recovers a grossly typical organization after allogenic hand transplantation. Curr. Biol., 18, 1530-1534. (p. 82, 107)

Fritsch, G. \& Hitzig, E. (1870). Über die elektrische erregbarkeit des grosshirns. Arch. Anat. Physiol. Med. Wiss., 37, 300-332. (p. 10) 
Galvani, L. (1791). De viribus electricitatis in motu musculari commentarius. Bono. Sci. Art. Acad. Comment., 7, 363-418. (p. 9)

Gamzu, E. \& Ahissar, E. (2001). Importance of temporal cues for tactile spatial-frequency discrimination. J Neurosci, 21, 7416-7427. (p. 86)

Georgopoulos, A. P., Kalaska, J. F., Caminiti, R., \& Massey, J. T. (1982). On the relations between the direction of two-dimensional arm movements and cell discharge in primate motor cortex. J Neurosci, 2, 1527-1537. (p. 5, 6)

Georgopoulos, A. P., Schwartz, A. B., \& Kettner, R. E. (1986). Neuronal population coding of movement direction. Science, 233, 1416-1419. (p. 5, 6)

Gilja, V., Chestek, C., Diester, I., Henderson, J., Deisseroth, K., \& Shenoy, K. V. (2011). Challenges and opportunities for next-generation intra-cortically based neural prostheses. IEEE Transactions on Biomedical Engineering. (p. 110)

Grandvalet, Y. (1998). Least absolute shrinkage is equivalent to quadratic penalization. Perspectives in Neural Computing, pp. 201-206. (p. 93)

Graziano, M. S. A., Cooke, D. F., \& Taylor, C. S. (2000). Coding the location of the arm by sight. Science, 290, 1782-1786. (p. 107)

Green, R. M. (1953). A Translation of Luigi Galvani’s De viribus electricitatis in motu musculari commentarius. Commentary on the Effect of Electricity on Muscular Motion. (Cambridge, MA: Elizabeth Licht). (p. 9)

Groenegress, C., Holzner, C., Guger, C., \& Slater, M. (2010). Effects of p3oo-based bci use on reported presence in a virtual environment. Presence: Teleoperators and Virtual Environments, 19. (p. 116)

Guenther, F. H., Brumberg, J. S., Wright, E. J., Nieto-Castanon, A., Tourville, J. A., Panko, M., Law, R., Siebert, S. A., Bartels, J. L., Andreasen, D. S., Ehirim, P., Mao, H., \& Kennedy, P. R. (2009). A wireless brain-machine interface for real-time speech synthesis. PLoS ONE, 4, e8218. (p. 9, 85)

Hales, W. B. (1945). Recording lissajous figures. J Acoust Soc Am, 16, 137-146. (p. 36)

Hatsopoulos, N. G., Joshi, J., \& O’Leary, J. G. (2004). Decoding continuous and discrete motor behaviors using motor and premotor cortical ensembles. J Neurophysiol, 92, 1165-1174. (p. 20)

Haykin, S. (2002). Adaptive Filter Theory. (Upper Saddle River, NJ: Prentice Hall), 4th edn. (p. 23)

Heming, E., Sanden, A., \& Kiss, Z. H. T. (2010). Designing a somatosensory neural prosthesis: percepts evoked by different patterns of thalamic stimulation. Journal of neural engineering, 7,064001 . (p. 12, 56) 
Histed, M. H., Bonin, V., \& Reid, R. C. (2009). Direct activation of sparse, distributed populations of cortical neurons by electrical microstimulation. Neuron, 63, 508-522. (p. 101, 110)

Hochberg, L. R., Serruya, M. D., Friehs, G. M., Mukand, J. A., Saleh, M., Caplan, A. H., Branner, A., Chen, D., Penn, R. D., \& Donoghue, J. P. (2006). Neuronal ensemble control of prosthetic devices by a human with tetraplegia. Nature, 442, 164-171. (p. 8)

Houweling, A. R. \& Brecht, M. (2008). Behavioural report of single neuron stimulation in somatosensory cortex. Nature, 451, 65-68. (p. 85)

Huber, D., Petreanu, L., Ghitani, N., Ranade, S., Hromádka, T., Mainen, Z., \& Svoboda, K. (2008). Sparse optical microstimulation in barrel cortex drives learned behaviour in freely moving mice. Nature, 451, 61-64. (p. 101)

James, T. W., Kim, S., \& Fisher, J. S. (2007). The neural basis of haptic object processing. Can J Exp Psychol, 61, 219-229. (p. 31, 59)

Jimbo, Y., Kasai, N., Torimitsu, K., Tateno, T., \& Robinson, H. P. C. (2003). A system for mea-based multisite stimulation. IEEE Transactions on Biomedical Engineering, 50, 241-248. (p. 109)

Johansson, R. S. \& Westling, G. (1984). Roles of glabrous skin receptors and sensorimotor memory in automatic control of precision grip when lifting rougher or more slippery objects. Exp Brain Res, 56, 550-564. (p. 59)

Kaczmarek, K., Webster, J., y Rita, P. B., \& Tompkins, W. (1991). Electrotactile and vibrotactile displays for sensory substitution systems. IEEE Transactions on Biomedical Engineering, 38, 1-16. (p. 59, 107)

Katz, D. \& Krueger, L. E. (1989). The world of touch. (Hillsdale, NJ: L. Erlbaum). (p. 59, 86)

Kennedy, P. R. \& Bakay, R. A. (1998). Restoration of neural output from a paralyzed patient by a direct brain connection. Neuroreport, 9, 1707-1711. (p. 8)

Keysers, C., Wicker, B., Gazzola, V., Anton, J.-L., Fogassi, L., \& Gallese, V. (2004). A touching sight: Sii/pv activation during the observation and experience of touch. Neuron, 42, 335-346. (p. 116)

Knaflitz, M. \& Merletti, R. (1988). Suppression of simulation artifacts from myoelectricevoked potential recordings. IEEE Trans Biomed Eng, 35, 758-763. (p. 109)

Kruger, L. ed. (1996). Pain and Touch. (San Diego, CA: Academic Press), 2nd edn. (p. 11)

Kübler, A., Kotchoubey, B., Kaiser, J., Wolpaw, J. R., \& Birbaumer, N. (2001). Brain-computer communication: unlocking the locked in. Psychological Bulletin, 127, 358-375. (p. 3, 8) 
Lebedev, M. A., Carmena, J. M., O’Doherty, J. E., Zacksenhouse, M., Henriquez, C. S., Principe, J. C., \& Nicolelis, M. A. L. (2005). Cortical ensemble adaptation to represent velocity of an artificial actuator controlled by a brain-machine interface. J Neurosci, 25, 4681-4693. (p. 23, 42, 66, 73, 75)

Lebedev, M. A., Denton, J. M., \& Nelson, R. J. (1994). Vibration-entrained and premovement activity in monkey primary somatosensory cortex. J Neurophysiol, 72, 1654-1673. (p. 72, 86)

Lebedev, M. A. \& Nicolelis, M. A. L. (2006). Brain-machine interfaces: past, present and future. Trends Neurosci., 29, 536-546. (p. 31, 32, 59, 79, 82, 85, 86, 106)

Lederman, S. J. (1974). Tactile roughness of grooved surfaces: the touching process and effects of macro- and microsurface structure. Perception \& Psychophysics, 16, 385-395. (p. 111, 113)

Lederman, S. J. \& Klatzky, R. L. (1987). Hand movements: a window into haptic object recognition. Cogn Psychol, 19, 342-368. (p. 66, 112)

Leuthardt, E. C., Schalk, G., Wolpaw, J. R., Ojemann, J. G., \& Moran, D. W. (2004). A braincomputer interface using electrocorticographic signals in humans. Journal of neural engineering, $1,63-71$. (p. 8 )

Li, Z., O’Doherty, J. E., Hanson, T. L., Lebedev, M. A., Henriquez, C. S., \& Nicolelis, M. A. L. (2009). Unscented kalman filter for brain-machine interfaces. PLoS ONE, 4, e6243. (p. $24,63,66,93,97)$

Liu, Y., Denton, J. M., \& Nelson, R. J. (2005). Neuronal activity in primary motor cortex differs when monkeys perform somatosensory and visually guided wrist movements. Exp Brain Res, 167, 571-586. (p. 72)

Loeb, G. E., Byers, C. L., Rebscher, S. J., Casey, D. E., Fong, M. M., Schindler, R. A., Gray, R. F., \& Merzenich, M. M. (1983). Design and fabrication of an experimental cochlear prosthesis. Medical \& biological engineering \& computing, 21, 241-254. (p. 11)

London, B. M., Jordan, L. R., Jackson, C. R., \& Miller, L. E. (2008). Electrical stimulation of the proprioceptive cortex (area 3 a) used to instruct a behaving monkey. IEEE Transactions on Neural Systems and Rehabilitation Engineering, 16, 32-36. (p. 57)

Marasco, P. D., Schultz, A. E., \& Kuiken, T. A. (2009). Sensory capacity of reinnervated skin after redirection of amputated upper limb nerves to the chest. Brain, 132, 1441-1448. (p. $12,60)$

Maravita, A., Spence, C., \& Driver, J. (2003). Multisensory integration and the body schema: close to hand and within reach. Curr. Biol., 13, R531-9. (p. 116) 
Marzullo, T. C., Lehmkuhle, M. J., Gage, G. J., \& Kipke, D. R. (2010). Development of closed-loop neural interface technology in a rat model: combining motor cortex operant conditioning with visual cortex microstimulation. IEEE Transactions on Neural Systems and Rehabilitation Engineering, 18, 117-126. (p. 13, 85)

McDonnell, M. D. \& Abbott, D. (2009). What is stochastic resonance? definitions, misconceptions, debates, and its relevance to biology. PLoS Comput Biol, 5, e1000348. (p. 102)

Merzenich, M. M., Schindler, D. N., \& White, M. W. (1974). Feasibility of multichannel scala tympani stimulation. Laryngoscope, 84, 1887-1893. (p. 11, 31, 85)

Moritz, C. T., Perlmutter, S. I., \& Fetz, E. E. (2008). Direct control of paralysed muscles by cortical neurons. Nature, 456, 639-642. (p. 7, 31, 57, 59)

Mountcastle, V. B., Steinmetz, M. A., \& Romo, R. (1990). Frequency discrimination in the sense of flutter: psychophysical measurements correlated with postcentral events in behaving monkeys. J Neurosci, 10, 3032-3044. (p. 86)

Musallam, S., Corneil, B. D., Greger, B., Scherberger, H., \& Andersen, R. A. (2004). Cognitive control signals for neural prosthetics. Science, 305, 258-262. (p. 6)

Mussa-Ivaldi, F. A., Alford, S. T., Chiappalone, M., Fadiga, L., Karniel, A., Kositsky, M., Maggiolini, E., Panzeri, S., Sanguineti, V., Semprini, M., \& Vato, A. (2010). New perspectives on the dialogue between brains and machines. Frontiers in neuroscience, 4, 44. (p. 85)

Neuburger, M. (1981). The Historical Development of Experimental Brain and Spinal Cord Physiology before Flourens. (Baltimore, MD: Johns Hopkins University Press). (p. 9)

Nicolelis, M. A. \& Chapin, J. K. (1994). Spatiotemporal structure of somatosensory responses of many-neuron ensembles in the rat ventral posterior medial nucleus of the thalamus. J Neurosci, 14, 3511-3532. (p. 5)

Nicolelis, M. A., Lin, R. C., Woodward, D. J., \& Chapin, J. K. (1993a). Dynamic and distributed properties of many-neuron ensembles in the ventral posterior medial thalamus of awake rats. Proc Natl Acad Sci USA, 90, 2212-2216. (p. 5)

Nicolelis, M. A., Lin, R. C., Woodward, D. J., \& Chapin, J. K. (1993b). Induction of immediate spatiotemporal changes in thalamic networks by peripheral block of ascending cutaneous information. Nature, 361, 533-536. (p. 5)

Nicolelis, M. A. L. (2001). Actions from thoughts. Nature, 409, 403-407. (p. 31)

Nicolelis, M. A. L. (2003). Brain-machine interfaces to restore motor function and probe neural circuits. Nat Rev Neurosci, 4, 417-422. (p. 31) 
Nicolelis, M. A. L., Baccala, L. A., Lin, R. C., \& Chapin, J. K. (1995). Sensorimotor encoding by synchronous neural ensemble activity at multiple levels of the somatosensory system. Science, 268, 1353-1358. (p. 5)

Nicolelis, M. A. L., Dimitrov, D. F., Carmena, J. M., Crist, R. E., Lehew, G., Kralik, J. D., \& Wise, S. P. (2003). Chronic, multisite, multielectrode recordings in macaque monkeys. Proc Natl Acad Sci USA, 100, 11041-11046. (p. 20, 87)

Nicolelis, M. A. L., Ghazanfar, A. A., Faggin, B. M., Votaw, S., \& Oliveira, L. (1997). Reconstructing the engram: simultaneous, multisite, many single neuron recordings. Neuron, 18, 529-537. (p. 6)

Nicolelis, M. A. L. \& Lebedev, M. A. (2009). Principles of neural ensemble physiology underlying the operation of brain-machine interfaces. Nat Rev Neurosci, 10, 530-540. (p. $1,3,59,85,86)$

Obeid, I., Veraart, C., \& Delbeke, J. (2010). Estimation of phosphene spatial variability for visual prosthesis applications. Artificial organs, 34, 358-365. (p. 11)

O’Doherty, J. E., Lebedev, M. A., Hanson, T. L., Fitzsimmons, N. A., \& Nicolelis, M. A. L. (2009). A brain-machine interface instructed by direct intracortical microstimulation. Front. Integr. Neurosci., 3, 20. (p. 14, 59, 73, 85, 86, 87, 89, 93, 99, 101, 105)

Patil, P. G., Carmena, J. M., Nicolelis, M. A. L., \& Turner, D. A. (2004). Ensemble recordings of human subcortical neurons as a source of motor control signals for a brain-machine interface. Neurosurgery, 55, 27-35; discussion 35-8. (p. 8)

Pei, Y.-C., Hsiao, S. S., Craig, J. C., \& Bensmaia, S. J. (2011). Neural mechanisms of tactile motion integration in somatosensory cortex. Neuron, 69, 536-547. (p. 111)

Penfield, W. \& Boldrey, E. (1937). Somatic motor sensory representation in the cerebral cortex of man as studied by electrical stimulation. Brain J Neurol, 60, 389-443. (p. 10, 54, $105,115)$

Penfield, W. \& Rasmussen, T. (1952). The Cerebral Cortex of Man. (New York, NY: Macmillan). (p. 10, 11)

Petersen, C. C. H., Hahn, T. T. G., Mehta, M., Grinvald, A., \& Sakmann, B. (2003). Interaction of sensory responses with spontaneous depolarization in layer $2 / 3$ barrel cortex. Proc Natl Acad Sci USA, 100, 13638-13643. (p. 113)

Peterson, N. R., Pisoni, D. B., \& Miyamoto, R. T. (2010). Cochlear implants and spoken language processing abilities: review and assessment of the literature. Restor Neurol Neurosci, 28, 237-250. (p. 85) 
Phillips, J. R. \& Johnson, K. O. (1981). Tactile spatial resolution. ii. neural representation of bars, edges, and gratings in monkey primary afferents. J Neurophysiol, 46, 1192-1203. (p. 86)

Pohlmeyer, E. A., Oby, E. R., Perreault, E. J., Solla, S. A., Kilgore, K. L., Kirsch, R. F., \& Miller, L. E. (2009). Toward the restoration of hand use to a paralyzed monkey: brain-controlled functional electrical stimulation of forearm muscles. PLoS ONE, 4, e5924. (p. 7)

Polikov, V. S., Tresco, P. A., \& Reichert, W. M. (2005). Response of brain tissue to chronically implanted neural electrodes. J Neurosci Methods, 148, 1-18. (p. 9)

Pons, T. P., Garraghty, P. E., Ommaya, A. K., Kaas, J. H., Taub, E., \& Mishkin, M. (1991). Massive cortical reorganization after sensory deafferentation in adult macaques. Science, $252,1857-1860$. (p. 82, 107)

Ramachandran, V.S. (1993). Behavioral and magnetoencephalographic correlates of plasticity in the adult human brain. Proc Natl Acad Sci USA, 90, 10413-10420.(p. 105)

Ranck, J. B. (1975). Which elements are excited in electrical stimulation of mammalian central nervous system: a review. Brain Res, 98, 417-440. (р. 110)

Ribot-Ciscar, E., Bergenheim, M., Albert, F., \& Roll, J.-P. (2003). Proprioceptive population coding of limb position in humans. Exp Brain Res, 149, 512-519. (p. 31, 59)

Richer, F., Martinez, M., Robert, M., Bouvier, G., \& Saint-Hilaire, J. M. (1993). Stimulation of human somatosensory cortex: tactile and body displacement perceptions in medial regions. Exp Brain Res, 93, 173-176. (p. 56, 60)

Rijpkema, H. \& Girard, M. (1991). Computer animation of knowledge-based human grasping. SIGGRAPH '91: Proceedings of the 18th annual conference on Computer graphics and interactive techniques. (p. 29)

Rolston, J. D., Gross, R. E., \& Potter, S. M. (2009). Neurorighter: closed-loop multielectrode stimulation and recording for freely moving animals and cell cultures. Conference proceedings : ... Annual International Conference of the IEEE Engineering in Medicine and Biology Society. IEEE Engineering in Medicine and Biology Society. Conference, 2009, 6489-6492. (p. 101, 109)

Romo, R., Hernández, A., Zainos, A., Brody, C. D., \& Lemus, L. (200o). Sensing without touching: psychophysical performance based on cortical microstimulation. Neuron, 26, 273-278. (p. 12, 85, 100)

Romo, R., Hernández, A., Zainos, A., \& Salinas, E. (1998). Somatosensory discrimination based on cortical microstimulation. Nature, 392, 387-390. (p. 12, 54, 55, 57, 60, 85, 100, 106) 
Rossi, L., Foffani, G., Marceglia, S., Bracchi, F., Barbieri, S., \& Priori, A. (2007). An electronic device for artefact suppression in human local field potential recordings during deep brain stimulation. Journal of neural engineering, 4, 96-106. (p. 109)

Salinas, E., Hernandez, A., Zainos, A., \& Romo, R. (2000). Periodicity and firing rate as candidate neural codes for the frequency of vibrotactile stimuli. J Neurosci, 20, 5503-5515. (p. 86)

Santaniello, S., Fiengo, G., Glielmo, L., \& Grill, W. M. (2011). Closed-loop control of deep brain stimulation: a simulation study. IEEE Transactions on Neural Systems and Rehabilitation Engineering, 19, 15-24. (p. 13)

Schaefer, M., Flor, H., Heinze, H.-J., \& Rotte, M. (2007). Morphing the body: illusory feeling of an elongated arm affects somatosensory homunculus. Neuroimage, 36, 700-705. (p. 116)

Schaefer, M., Noennig, N., Heinze, H.-J., \& Rotte, M. (2006). Fooling your feelings: artificially induced referred sensations are linked to a modulation of the primary somatosensory cortex. Neuroimage, 29, 67-73. (p. 116)

Schmidt, E., Bak, M. J., Hambrecht, F. T., Kufta, C. V., O’Rourke, D. K., \& Vallabhanath, P. (1996). Feasibility of a visual prosthesis for the blind based on intracortical microstimulation of the visual cortex. Brain, 119 ( Pt 2), 507-522. (p. 113)

Schmidt, E., Bak, M. J., McIntosh, J. S., \& Thomas, J. S. (1977). Operant conditioning of firing patterns in monkey cortical neurons. Exp Neurol, 54, 467-477. (p. 4)

Schmidt, E., McIntosh, J. S., Durelli, L., \& Bak, M. J. (1978). Fine control of operantly conditioned firing patterns of cortical neurons. Exp Neurol, 61, 349-369. (p. 4)

Schwartz, A. B., Cui, X. T., Weber, D. J., \& Moran, D. W. (2006). Brain-controlled interfaces: movement restoration with neural prosthetics. Neuron, 52, 205-220. (p. 31)

Scott, R. N., Brittain, R. H., Caldwell, R. R., Cameron, A. B., \& Dunfield, V. A. (1980). Sensory-feedback system compatible with myoelectric control. Medical \& biological engineering \& computing, 18, 65-69. (p. 59, 108)

Serruya, M. D., Hatsopoulos, N. G., Paninski, L., Fellows, M. R., \& Donoghue, J. P. (2002). Instant neural control of a movement signal. Nature, 416, 141-142. (p. 6, 31, 57)

Shoham, S., Halgren, E., Maynard, E. M., \& Normann, R. A. (2001). Motor-cortical activity in tetraplegics. Nature, 413, 793. (p. 8)

Simões-Franklin, C., Whitaker, T. A., \& Newell, F. N. (2010). Active and passive touch differentially activate somatosensory cortex in texture perception. Hum Brain Mapp. (p. 102) 
Sinclair, R. \& Burton, H. (1988). Responses from area 3 b of somatosensory cortex to textured surfaces during active touch in primate. Somatosens Res, 5, 283-310. (p. 102)

Sinclair, R. J. \& Burton, H. (1991). Tactile discrimination of gratings: psychophysical and neural correlates in human and monkey. Somatosens Mot Res, 8, 241-248. (p. 86)

Sinclair, R. J., Pruett, J. R., \& Burton, H. (1996). Responses in primary somatosensory cortex of rhesus monkey to controlled application of embossed grating and bar patterns. Somatosens Mot Res, 13, 287-306. (p. 86)

Song, Y.-K., Borton, D. A., Park, S., Patterson, W. R., Bull, C. W., Laiwalla, F., Mislow, J., Simeral, J. D., Donoghue, J. P., \& Nurmikko, A. V. (2009). Active microelectronic neurosensor arrays for implantable brain communication interfaces. IEEE Transactions on Neural Systems and Rehabilitation Engineering, 17, 339-345. (p. 12)

Srinivasan, M. A. \& Dandekar, K. (1996). An investigation of the mechanics of tactile sense using two-dimensional models of the primate fingertip. J Biomech Eng, 118, 48-55. (p. 110)

Suminski, A. J., Tkach, D. C., Fagg, A. H., \& Hatsopoulos, N. G. (2010). Incorporating feedback from multiple sensory modalities enhances brain-machine interface control. J Neurosci, 30, 16777-16787. (p. 85)

Talwar, S. K., Xu, S., Hawley, E. S., Weiss, S. A., Moxon, K. A., \& Chapin, J. K. (2002). Rat navigation guided by remote control. Nature, 417, 37-38. (p. 12, 57, 85)

Taylor, D. M., Helms-Tillery, S. I., \& Schwartz, A. B. (2002). Direct cortical control of 3d neuroprosthetic devices. Science, 296, 1829-1832. (p. 6, 31, 41, 57, 59)

Tkach, D. C., Reimer, J., \& Hatsopoulos, N. G. (2007). Congruent activity during action and action observation in motor cortex. J Neurosci, 27, 13241-13250. (p. 42)

Van Doren, C. L. (1989). A model of spatiotemporal tactile sensitivity linking psychophysics to tissue mechanics. J Acoust Soc Am, 85, 2065-208o. (p. 110)

Velliste, M., Perel, S., Spalding, M. C., Whitford, A. S., \& Schwartz, A. B. (2008). Cortical control of a prosthetic arm for self-feeding. Nature, 453, 1098-1101. (p. 7, 31, 57, 59)

Venkatraman, S. \& Carmena, J. (2011). Active sensing of target location encoded by cortical microstimulation. IEEE Transactions on Neural Systems and Rehabilitation Engineering. (p. 13, 85)

Venkatraman, S., Elkabany, K., Long, J. D., Yao, Y., \& Carmena, J. M. (2009). A system for neural recording and closed-loop intracortical microstimulation in awake rodents. IEEE Transactions on Biomedical Engineering, 56, 15-22. (p. 109) 
Vidal-Verdú, F. \& Hafez, M. (2007). Graphical tactile displays for visually-impaired people. IEEE Transactions on Neural Systems and Rehabilitation Engineering, 15, 119-130. (p. 56)

von Bonin, G. (1960). Some Papers on the Cerebral Cortex. (Springfield, IL: Charles C. Thomas). (p. 10)

Wagenaar, D. A. \& Potter, S. M. (2002). Real-time multi-channel stimulus artifact suppression by local curve fitting. J Neurosci Methods, 120, 113-120. (p. 109)

Ward, L. M., MacLean, S. E., \& Kirschner, A. (2010). Stochastic resonance modulates neural synchronization within and between cortical sources. PLoS ONE, 5, e14371. (p. 102)

Wessberg, J., Stambaugh, C. R., Kralik, J. D., Beck, P. D., Laubach, M., Chapin, J. K., Kim, J., Biggs, S. J., Srinivasan, M. A., \& Nicolelis, M. A. L. (2000). Real-time prediction of hand trajectory by ensembles of cortical neurons in primates. Nature, 408, 361-365. (p. 6, 23, 31, $39,41,57,59)$

Wise, K. D., Angell, J. B., \& Starr, A. (1970). An integrated-circuit approach to extracellular microelectrodes. IEEE Trans Biomed Eng, 17, 238-247. (p. 5)

Witney, A. G., Wing, A., Thonnard, J.-L., \& Smith, A. M. (2004). The cutaneous contribution to adaptive precision grip. Trends Neurosci., 27, 637-643. (p. 31)

Zhang, F., Wang, L.-P., Brauner, M., Liewald, J. F., Kay, K., Watzke, N., Wood, P. G., Bamberg, E., Nagel, G., Gottschalk, A., \& Deisseroth, K. (2007). Multimodal fast optical interrogation of neural circuitry. Nature, 446, 633-639. (p. 82, 101, 110)

Zhang, X., Roppolo, J. R., de Groat, W. C., \& Tai, C. (2006). Simulation analysis of conduction block in myelinated axons induced by high-frequency biphasic rectangular pulses. IEEE Transactions on Biomedical Engineering, 53, 1433-1436. (p. 101)

Zhou, Y. D. \& Fuster, J. M. (1996). Mnemonic neuronal activity in somatosensory cortex. Proc Natl Acad Sci USA, 93, 10533-10537. (p. 73) 


\title{
Biography
}

\author{
Joseph Emmanuel O’Doherty \\ Born: 26 May 1979 \\ Mobile, Alabama, USA
}

\section{Education}

Ph.D., Biomedical Engineering, May 2011

Duke University, Durham, NC

B.S. Physics, Magna Cum Laude, May 2001

East Carolina University, Greenville, NC

\section{Awards}

Graduate Student Extended Abstract Award, 2010

Biomedical Engineering Society

Workshop Travel Award, 2010

IEEE Engineering in Medicine \& Biology Society

Burroughs Welcome Fellowship (declined), 2001

UNC Graduate School

James Fenly Spear, Jr. Memorial Award, 2001

East Carolina University

\section{Refereed Publications}

O’Doherty, J.E., Lebedev, M.A., Hanson, T.L., Fitzsimmons, N.A. \& M.A.L. Nicolelis. (2009). A brain-machine interface instructed by direct intracortical microstimulation. Frontiers in Integrative Neuroscience, 3, 20. doi: 10.3389/neuro.07.020.2009

Li, Z., O’Doherty, J.E., Hanson, T.L., Lebedev, M.A., Henriquez, C.S. \& M.A.L. Nicolelis. (2009). Unscented Kalman filter for brain-machine interfaces. PLoS ONE, 4(7), e6243. doi: 10.1371/journal.pone.0006243 
Lebedev, M.A., O’Doherty, J.E. \& M.A.L. Nicolelis. (2008). Decoding of temporal intervals from cortical ensemble activity. The Journal of Neurophysiology, 99(1), 166-186. doi: 10.1152/jn.00734.2007

Zacksenhouse, M., Lebedev, M.A., Carmena, J.M., O’Doherty, J.E., Henriquez, C.S. \& M.A.L. Nicolelis. (2007). Cortical modulations increase in early sessions with brain-machine interface. PLoS ONE, 2(7), e619. doi: 10.1371/journal.pone.0000619

Lebedev, M.A., Carmena, J.M., O’Doherty, J.E., Zacksenhouse, M., Henriquez, C.S., Principe, J.C. \& M.A.L. Nicolelis. (2005). Cortical ensemble adaptation to represent actuators controlled by a brain machine interface. The Journal of Neuroscience, 25(19), 4681-4693. doi: 10.1523/JNEUROSCI.4088-04.2005

Carmena, J.M., Lebedev, M.A., Crist, R.E., O’Doherty, J.E., Santucci, D.M., Dimitrov, D.F., Patil, P.G., Henriquez, C.S. \& M.A.L. Nicolelis. (2003). Learning to control a brain-machine interface for reaching and grasping by primates. PLoS Biology, 1(2), e42. doi: 10.1371/journal.pbio.0000042

Gumber S.C. \& J.E. O’Doherty. Digestive disease resources on the Internet. (1999). The American Journal of Gastroenterology, 94(8), 2022-2032. doi: 10.1016/Sooo2-9270(99)00329-9

\section{Book Chapters}

Hanson, T.L., Fitzsimmons, N.A. \& J.E. O’Doherty. (2007). Technology for Multielectrode MicroStimulation of Brain Tissue, in Methods for Neural Ensemble Recordings, Second Edition. Nicolelis, M.A.L., Editor. CRC Press.

\section{Manuscripts Under Peer Review (at time of writing)}

O’Doherty, J.E., Lebedev, M.A., Ifft, P.J., Zhuang, K.Z., Shokur, S. Bleuler, H. \& M.A.L. Nicolelis. Enacting motor intentions and tactile sensations through a brain-machine-brain interface.

O’Doherty, J.E., Lebedev, M.A., Li, Z. \& M.A.L. Nicolelis. Towards a brainmachine-brain interface: active touch using randomly patterned intracortical microstimulation.

Lebedev, M.A., Tate, A.J., Hanson, T.L., Li, Z., O'Doherty, J.E., Winans, J.A., Ifft, P.J., Zhuang, K.Z., Fitzsimmons, N.A., Schwarz, D.A., Fuller, A.M., An, J.H. \& M.A.L. Nicolelis. Future developments in brain-machine interface research.

Li, Z., O’Doherty, J.E., Lebedev, M.A. and M.A.L. Nicolelis. Adaptive decoding for brain-machine interfaces using Bayesian regression updates of parameters. 